\title{
A formal system for Euclid's Elements
}

\author{
Jeremy Avigad, Edward Dean, and John Mumma*
}

May 4, 2009

\begin{abstract}
We present a formal system, $E$, which provides a faithful model of the proofs in Euclid's Elements, including the use of diagrammatic reasoning.
\end{abstract}

\section{Contents}

1 Introduction 2

2 Characterizing the Elements 4

2.1 Examples of proofs in the Elements . . . . . . . . . . . . 5

2.2 The use of diagrams . . . . . . . . . . . . . . 7

2.3 The problem of ensuring generality . . . . . . . . . . 8

2.4 The logical form of proofs in the Elements . . . . . . . . . . 10

2.5 Nondegeneracy assumptions . . . . . . . . . . . . . . . . 11

2.6 Our methodology . . . . . . . . . . . . . . . . . . . . 12

3 The formal system $E \quad 13$

3.1 The language of $E \ldots \ldots \ldots \ldots \ldots \ldots$

3.2 Proofs in $E \ldots \ldots \ldots \ldots \ldots \ldots \ldots$

3.3 Construction rules $\ldots \ldots \ldots \ldots \ldots$

3.4 Diagrammatic inferences . . . . . . . . . . . . . . . . . . 24

3.5 Metric inferences . . . . . . . . . . . . . . . . . . 28

3.6 Transfer inferences . . . . . . . . . . . . . . . . . . . . . 30

3.7 Superposition . . . . . . . . . . . . . . . . . . . . . . . 31

3.8 The notion of a "direct consequence" . . . . . . . . . . . 33

${ }^{*}$ We are especially indebted to Ken Manders, whose work and encouragement set us on our way. We would also like to thank Jesse Alama, Alan Baker, Michael Beeson, Karine Chemla, Annalisa Coliva, Mic Detlefsen, Harvey Friedman, Mark Goodwin, Jeremy Gray, Jeremy Heis, Anthony Jones, Danielle Macbeth, Penelope Maddy, Paolo Mancosu, Henry Mendell, Marco Panza, Jan von Plato, Vaughan Pratt, Dana Scott, Lisa Shabel, Stewart Shapiro, Wilfried Sieg, Neil Tennant, Freek Wiedijk, and an anonymous referee for helpful comments, criticisms, corrections, and suggestions; and we apologize to any others who also deserve our thanks but have been inadvertently overlooked. Avigad's work has been partially supported by NSF grant DMS-0700174 and a grant from the John Templeton Foundation. 
4 Comparison with the Elements 40

4.1 Language . . . . . . . . . . . . . . . . . . 41

4.2 Examples of proofs in $E \ldots \ldots \ldots \ldots \ldots \ldots$

4.3 Departures from the Elements . . . . . . . . . . . . . . 48

4.4 Euclid's postulates and common notions . . . . . . . . . . 51

4.5 Additional proofs . . . . . . . . . . . . . . . 52

5 Completeness 54

5.1 The semantics of ruler-and-compass constructions . . . . . . . 54

5.2 Tarski's system . . . . . . . . . . . . . . . . . 56

5.3 Translating $E$ to $T \ldots \ldots \ldots \ldots \ldots \ldots \ldots$

5.4 Interpreting $T$ in $E \ldots \ldots \ldots \ldots \ldots$

6 Implementation 7

7 Conclusions 74

7.1 Summary of results . . . . . . . . . . . . . . . 74

7.2 Questions and related work . . . . . . . . . 75

7.3 Broader issues . . . . . . . . . . . . . . . . . 76

\section{Introduction}

For more than two millennia, Euclid's Elements was viewed by mathematicians and philosophers alike as a paradigm of rigorous argumentation. But the work lost some of its lofty status in the nineteenth century, amidst concerns related to the use of diagrams in its proofs. Recognizing the correctness of Euclid's inferences was thought to require an "intuitive" use of these diagrams, whereas, in a proper mathematical argument, every assumption should be spelled out explicitly. Moreover, there is the question as to how an argument that relies on a single diagram can serve to justify a general mathematical claim: any triangle one draws will, for example, be either acute, right, or obtuse, leaving the same intuitive faculty burdened with the task of ensuring that the argument is equally valid for all triangles 1 Such a reliance on intuition was therefore felt to fall short of delivering mathematical certainty.

Without denying the importance of the Elements, by the end of the nineteenth century the common attitude among mathematicians and philosophers was that the appropriate logical analysis of geometric inference should be cast in terms of axioms and rules of inference. This view was neatly summed up by Leibniz more than two centuries earlier:

...it is not the figures which furnish the proof with geometers, though the style of the exposition may make you think so. The force of the demonstration is independent of the figure drawn, which is drawn only to facilitate the knowledge of our meaning, and to fix

\footnotetext{
${ }^{1}$ The question was raised by early modern philosophers from Berkeley [4, Section 16] to Kant [24, A716/B744]. See [17, 20, 40, 52, 53, 54] for discussions of the philosophical concerns.
} 
the attention; it is the universal propositions, i.e. the definitions, axioms, and theorems already demonstrated, which make the reasoning, and which would sustain it though the figure were not there. 27. p. 403]

This attitude gave rise to informal axiomatizations by Pasch [46], Peano [4], and Hilbert 22] in the late nineteenth century, and Tarski's formal axiomatization 57] in the twentieth.

Proofs in these axiomatic systems, however, do not look much like proofs in the Elements. Moreover, the modern attitude belies the fact that for over two thousand years Euclidean geometry was a remarkably stable practice. On the consensus view, the logical gaps in Euclid's presentation should have resulted in vagueness or ambiguity as to the admissible rules of inference. But, in practice, they did not; mathematicians through the ages and across cultures could read, write, and communicate Euclidean proofs without getting bogged down in questions of correctness. So, even if one accepts the consensus view, it is still reasonable to seek some sort of explanation of the success of the practice.

Our goal here is to provide a detailed analysis of the methods of inference that are employed in the Elements. We show, in particular, that the use of diagrams in a Euclidean proof is not soft and fuzzy, but controlled and systematic, and governed by a discernible logic. This provides a sense in which Euclid's methods are more rigorous than the modern attitude suggests.

Our study draws on an analysis of Euclidean reasoning due to Ken Manders [32, who distinguished between two types of assertions that are made of the geometric configurations arising in Euclid's proofs. The first type of assertion describes general topological properties of the configuration, such as incidence of points and lines, intersections, the relative position of points along a line, or inclusions of angles. Manders called these co-exact attributions, since they are stable under perturbations of the diagram; below, we use the term "diagrammatic assertions" instead. The second type includes things like congruence of segments and angles, and comparisons between linear or angular magnitudes. Manders called these exact attributions, because they are not stable under small variations, and hence may not be adequately represented in a figure that is roughly drawn. Below, we use the term "metric assertions" instead. Inspecting the proofs in the Elements, Manders observed that the diagrams are only used to record and infer co-exact claims; exact claims are always made explicit in the text. For example, one might infer from the diagram that a point lies between two others on a line, but one would never infer the congruence of two segments without justifying the conclusion in the text. Similarly, one cannot generally infer, from inspecting two angles in a diagram, that one is larger than the other; but one can draw this conclusion if the diagram "shows" that the first is properly contained in the second.

Below, we present a formal axiomatic system, $E$, which spells out precisely what inferences can be "read off" from the diagram. Our work builds on Mumma's $\mathrm{PhD}$ thesis [39, which developed such a diagram-based system, which he called Eu. In Mumma's system, diagrams are bona-fide objects, which 
are introduced in the course of a proof and serve to license inferences. Mumma's diagrams are represented by geometric objects on a finite coordinate grid. However, Mumma introduced a notion of "equivalent diagrams" to explain how one can apply a theorem derived from a different diagram that nonetheless bears the same diagrammatic information. Introducing an equivalence relation in this way suggests that, from a logical perspective, what is really relevant to the proof is the equivalence class of all the diagrams that bear the same information. We have thus chosen a more abstract route, whereby we identify the "diagram" with the co-exact information that the physical drawing is supposed to bear. Nathaniel Miller's PhD dissertation [35. provides another formal system for diagrammatic reasoning, along these lines, employing "diagrams" that are graph-theoretic objects subject to certain combinatorial constraints.

Both Mumma and Miller address the issue of how reasoning based on a particular diagram can secure general conclusions, though they do so in different ways. In Miller's system, when a construction can result in topologically distinct diagrammatic configurations, one is required to consider all the cases, and show that the desired conclusion is warranted in each. In contrast, Mumma stipulated general rules, based on the particulars of the construction, that must be followed to ensure that the facts read off from the particular diagram are generally valid. Our formulation of $E$ derives from this latter approach, which, we argue below, is more faithful to Euclidean practice.

Moreover, we show that our proof system is sound and complete for a standard semantics of "ruler-and-compass constructions," expressed in modern terms. Thus, our presentation of $E$ is accompanied by both philosophical and mathematical claims: on the one hand, we claim that our formal system accurately models many of the key methodological features that are characteristic of the proofs found in books I through IV of the Elements; and, on the other hand, we claim that it is sound and complete for the appropriate semantics.

The outline of this paper is as follows. In Section 2, we begin with an informal discussion of proofs in the Elements, calling attention to the particular features that we are trying to model. In Section 3 , we describe the formal system, $E$, and specify its language and rules of inference. In Section 4 we justify the claim that our system provides a faithful model of the proofs in the Elements, calling attention to points of departure as well as points of agreement. In Section [5, we show that our formal system is sound and complete with respect to ruler-andcompass constructions. In Section 6, we discuss ways in which contemporary methods of automated reasoning can be used to implement a proof checker

that can mechanically verify proofs in our system. Finally, in Section 7 , we summarize our findings, and indicate some questions and issues that are not addressed in our work.

\section{Characterizing the Elements}

In this section, we clarify the claim that our formal system is more faithful to the Elements than other axiomatic systems, by describing the features of the 
Elements that we take to be salient.

\subsection{Examples of proofs in the Elements}

To support our discussion, it will be helpful to have two examples of Euclidean proofs at hand.

Proposition I.10. To bisect a given finite straight line.

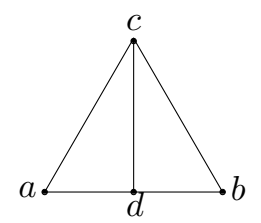

Proof. Let $a b$ be the given finite straight line.

It is required to bisect the finite straight line $a b$.

Let the equilateral triangle $a b c$ be constructed on it [I.1], and let the angle $a c b$ be bisected by the straight line $c d$. [I.9]

I say that the straight line $a b$ is bisected at the point $d$.

For, since $a c$ is equal to $c b$, and $c d$ is common, the two sides $a c, c d$ are equal the two sides $b c, c d$ respectively; and the angle $a c d$ is equal to the angle $b c d$; therefore the base $a d$ is equal to the base $b d$. [I.4]

Therefore the given finite straight line $a b$ has been bisected at $d$.

Q.E.F.

This is Proposition 10 of Book I of the Elements. All our references to the Elements refer to the Heath translation [16, though we have replaced uppercase labels for points by lower-case labels in the proof, to match the description of our formal system, $E$.

As is typical in the Elements, the initial statement of the proposition is stated in something approximating natural language. A more mathematical statement of the proposition is then given in the opening lines of the proof. The annotations in brackets refer back to prior propositions, so, for example, the third sentence of the proof refers to Propositions 1 and 9 of Book I. Notice that what it means for a point $d$ to "bisect" the finite segment $a b$ can be analyzed into topological and metric components: we expect $d$ to lie on the same line as $a$ and $b$, and to lie between $a$ and $b$ on that line; and we expect that the length of the segment from $a$ to $b$ is equal to the length of the segment from $b$ to $d$. Only the last part of the claim is made explicit in the text; the other two facts are implicit in the diagram.

In his fifth century commentary on the first book of the Elements, Proclus divided Euclid's propositions into two groups: "problems," which assert that a construction can be carried out, or a diagram expanded, in a certain way; and "theorems," which assert that certain properties are essential to a given diagram (see [36, pp. 63-67], or [16, vol. I, pp. 124-129]). Euclid himself marks the distinction by ending proofs of problems with the phrase "that which it 
was required to do" (abbreviated by "Q.E.F.," for "quod erat faciendum," by Heath); and ending proofs of theorems with the phrase "that which it was required to prove" (abbreviated by "Q.E.D.," for "quod erat demonstratum"). Proposition I.10 calls for the construction of a point bisecting the line, and so the proof ends with "Q.E.F."

Proposition I.16. In any triangle, if one of the sides be produced, then the exterior angle is greater than either of the interior and opposite angles.

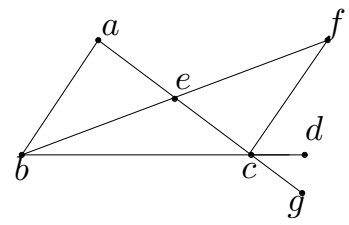

Proof. Let $a b c$ be a triangle, and let one side of it $b c$ be produced to $d$.

I say that the exterior angle $a c d$ is greater than either of the interior and opposite angles $c b a, b a c$.

Let $a c$ be bisected at $e$ [I.10],

and let be be joined and produced in a straight line to $f$;

Let $e f$ be made equal to be [I.3],

let $f c$ be joined, [Post.1]

and let $a c$ be drawn through to $g$. [Post.2]

Then, since $a e$ is equal to $e c$, and be to $e f$, the two sides $a e, e b$ are equal the two sides $c e$, ef respectively; and the angle $a e b$ is equal to the angle $f e c$, for they are vertical angles. [I.15]

Therefore the base $a b$ is equal to the base $f c$, the triangle $a b e$ is equal to the triangle $c f e$, and the remaining angles equal the remaining angles respectively, namely those which the equal sides subtend; [I.4] therefore the angle bae is equal to the angle ecf.

But the angle ecd is greater than the angle ecf; [C.N.5]

therefore the angle acd is greater than the angle bae.

Similarly also, if $b c$ be bisected, the angle $b c g$, that is, the angle $a c d$ [I.15], can be proved greater than the angle $a b c$ as well.

Therefore etc.

Q.E.D.

Here, the abbreviation "Post." in brackets refers to Euclid's postulates, while the abbreviation "C.N." refers to the common notions. Notice that the proposition assumes that the triangle is nondegenerate. Later on, Euclid will prove the stronger Proposition I.32, which shows the the exterior angle acd is exactly equal to the sum of the interior and opposite angles $c b a$ and bac. But to do that, he has to develop properties of parallel lines, for which the current proposition is needed.

In both cases, after stating the theorem, the proofs begin with a construction phrase (kataskeue), in which new objects are introduced into the diagram. This is followed by the deduction phase (apodeixis), where the desired conclusions 
are drawn. The demonstration phase is, for the most part, devoted towards registering metric information, that is, equalities and inequalities between various magnitudes. But some of the inferences depend on the diagrammatic configuration. For example, seeing that angles $a e b$ and $f e c$ are equal in the second proof requires checking the diagram to see that they are vertical angles. Similarly, seeing that ecd is greater than ecf is warranted by common notion 5 , "the whole is greater than the part," requires checking the diagram to confirm that $e c f$ is indeed contained in $e c d$.

\subsection{The use of diagrams}

The most salient feature of the Elements is the fact that diagrams play a role in the arguments. But what, exactly, does this mean?

Our first observation is that whatever role the diagram plays, it is inessential to the communication of the proof. In fact, data on the early history of the text of the Elements is meager, and there is no chain linking our contemporary diagrams with the ones that Euclid actually drew; it is likely that, over the years, diagrams were often reconstructed from the text (see Netz [44]). But a simple experiment offers more direct support for our claim. If you cover up the diagrams and reread the proofs in the last section, you will find that it is not difficult to reconstruct the diagram. Occasionally, important details are only represented in the diagram and not the text; for example, in the proof of Proposition I.10, the text does not indicate that $d$ is supposed to mark the intersection of the angle bisector and the opposite side of the triangle. But there is no reason why it couldn't; for example, we could replace the second sentence with the following one:

Let the equilateral triangle $a b c$ be constructed on it, let the angle $a c b$ be bisected by the straight line $L$, and let $d$ be the intersection of $L$ and $a b$.

The fact that minor changes like this render it straightforward to construct an adequate diagram suggests that the relevant information can easily be borne by the text.

But, to continue the experiment, try reading these proofs, or any of Euclid's proofs, without the diagram, and without drawing a diagram. You will likely finding yourself trying to imagine the diagram, to "see" that the ensuing diagrammatic claims are justified. So even if, in some sense, the text-based version of the proof is self-contained, there is something about the proof, and the tasks we need to perform to understand the proof, that makes it "diagrammatic."

To make the point clear, consider the following example:

Let $L$ be a line. Let $a$ and $b$ be points on $L$, and let $c$ be between $a$ and $b$. Let $d$ be between $a$ and $c$, and let $e$ be between $c$ and $b$. Is $d$ necessarily between $a$ and $e$ ?

Once again, it is hard to make sense of the question without drawing a diagram or picturing the situation in your mind's eye; but doing so should easily convince 
you that the answer is "yes." With the diagram in place, there is nothing more that needs to be said. The inference is immediate, whether or not we are able to cite the axioms governing the betweenness predicate that would be used to justify the assertion in an axiomatic proof system.

A central goal of this paper is to analyze and describe these fundamental diagrammatic inferences. In doing so, we do not attempt to explain why it is easier for us to verify these inferences with a physical diagram before us, nor do we attempt to explain the social or historical factors that made such inferences basic to the Elements. In other words, in analyzing the Elements, we adopt a methodological stance which focuses on the logical structure of the proofs while screening off other important issues. We return to a discussion of this in Section 2.6.

\subsection{The problem of ensuring generality}

On further reflection, the notion of a diagrammatic inference becomes puzzling. Consider the following example:

Let $a$ and $b$ be distinct points, and let $L$ be the line through $a$ and $b$. Let $c$ and $d$ be points on opposite sides of $L$, and let $M$ be the line through $c$ and $d$. Let $e$ be the intersection of $L$ and $M$. Is $e$ necessarily between $c$ and $d$ ?

Drawing a diagram, or picturing the situation in your mind's eye, should convince you that the answer is "yes," based on an "intuitive" understanding of the concepts involved:

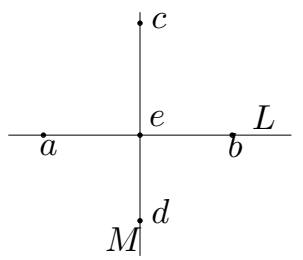

In fact, a diagrammatic inference was even implicit in the instruction "let $e$ be the intersection of $L$ and $M$," namely, in seeing that $L$ and $M$ necessarily intersect.

So far, all is well. But now suppose we replace the last question in the example with the following:

Is $e$ necessarily between $a$ and $b$ ?

Consulting our diagram, we should perhaps conclude that the answer is "yes." But that is patently absurd; we could easily have drawn the diagram to put $e$ anywhere along $L$. Neither Euclid nor any competent student of Euclidean geometry would draw the invalid inference. Thus any respectable notion of "diagrammatic inference" has to sanction the first inference in our example, but bar the second. 
There are two morals to be extracted from this little exercise. The first is that, however the diagram functions in a Euclidean proof, using the diagram is not simply a matter of reading off features found in the physical instantiation. Any way of drawing the diagram will give $e$ a position relative to $a$ and $b$, but none of them can be inferred from the givens. The physical instance of the diagram thus serves as a token, or artifact, that is intended to be used in certain ways; understanding the role of the diagram necessarily involves understanding the intended use ${ }^{2}$

The second moral is that the physical instance of the diagram, taken out of context, does not bear all the relevant inferential data. In the example above, the diagram is symmetric: if we rotate the diagram a quarter turn and switch the order of the questions, the new diagram and questionnaire differs from the previous one only by the labels of the geometric objects; but whereas "yes" and then "no" are the correct answers to the first set of questions, "no" and then "yes" are the correct answers to the second. What this means is that the inferences that we are allowed to perform depend not just on the illustration, but also on the preamble; that is, the inference depends on knowing the construction that the diagram is supposed to illustrate. Hence, understanding the role of the diagram in Euclidean practice also involves understanding how the details of the construction bear upon the allowable inferences.

In Nathaniel Miller's formal system for Euclidean geometry [35, every time a construction step can give rise to different topological configurations, the proof requires a case split across all the possible configurations. His system provides a calculus by which one can determine (an upper bound on) all the realizable configurations (and systematically rule out some of the configurations that are not realizable). This can result in a combinatorial explosion of cases, and Miller himself concedes that it can be difficult to work through them all. (See also Mumma's review [40.) Thus, although Miller's system is sound for the intended semantics and may be considered "diagrammatic" in nature, it seems far removed from the Elements, where such exhaustive case splits are nowhere to be found. (We will, however, have a lot more to say about the case distinctions that do appear in the Elements; see Sections 3.8 and 4.3 .)

Mumma's original proof system, $E u$ [39, 38, used a different approach. Although proofs in $E u$ are based on particular diagrams, not every feature found in a particular diagram can be used in the proof. Rather, one can only use those features of the diagram that are guaranteed to hold generally, given the diagram's construction. Mumma's system therefore includes precise rules that determine when a feature has this property. Our system, $E$, pushes the level of abstraction one step further: in $E$ the diagram is nothing more than the

\footnotetext{
${ }^{2}$ Danielle Macbeth [29] has characterized this sort of diagram use in terms of the Gricean distinction between "natural" and "non-natural" meaning. Manders [32] underscores this point by observing that Euclidean diagrams are used equally well in reductio proofs, where the conclusion is that the illustrated configuration cannot exist. One finds a nice example of this in Proposition 10 of Book III, which shows that two distinct circles cannot intersect in more than two points. Clearly, in cases like this, the diagram does not serve as a "literal" or direct representation of the relevant configuration.
} 
collection of generally valid diagrammatic features that are guaranteed by the construction. In other words, given the construction in the example above, we identify the diagram with the information provided by the construction - that $a$ and $b$ are distinct points, $L$ is a line, $a$ is on $L, b$ is on $L, c$ and $d$ are on opposite sides of $L$, and so on - and all the direct diagrammatic consequences of these data. This requires us to spell out the notion of a "direct diagrammatic consequence," which is exactly what we do in Section 3.8 .

\subsection{The logical form of proofs in the Elements}

It is commonly noted that Euclid's proofs are constructive, in the sense that existence assertions are established by giving explicit constructions. One would therefore not expect Euclidean reasoning to use the full range of classical firstorder logic, which allows nonconstructive existence proofs, but, rather, a suitably constructive fragment.

In fact, when one surveys the proofs in the Elements, one is struck by how little logic is involved, by modern standards. Go back to the examples in Section 2.1 and count the instances of logical staples like "every," "some," "or," and "if ...then." The results may surprise you.

Of course, the statements of the two propositions are best modeled with a universal quantifier: we can read Proposition I.10 as the assertion that "any finite straight line can be bisected" and Proposition I.16 begins with the words "any triangle." Furthermore, there is an existential quantifier implicit in the statement of Proposition I.10, which, in modern terms, might be expressed "for every finite straight line, there is a point that bisects it." In modern terms, it is the existential quantifier implicit in the statement of Proposition I.10 that makes this proposition a "problem" in Proclus' terminology. There is no such quantifier implicit in Proposition I.16, which is therefore a "theorem."

Thus, in a Euclidean proposition, an explicit or implicit universal quantifier serves to set forth the givens, and, if the proposition is a "problem," an existential statement is used to specify the properties of the objects to be constructed. What is remarkable is that these are the only quantifiers one finds in the text; the proof itself is purely quantifier-free. Not only that; the proof is virtually logic free. A construction step introduces new objects meeting certain specifications; for example, the third line of the proof of Proposition I.10 introduces an equilateral triangle. We will see that in our formalization, the specification can always be described as a list of atomic formulas and their negations. Other lines in a Euclidean proof simply make atomic or negated atomic statements, like "the base $a d$ is equal to the base $b d$," sometimes chained together with the word "and."

In other words, Euclidean proofs do little more than introduce objects satisfying lists of atomic (or negation atomic) assertions, and then draw further atomic (or negation atomic) conclusions from these, in a simple linear fashion. There are two minor departures from this pattern. Sometimes a Euclidean proof involves a case split; for example, if $a b$ and $c d$ are unequal segments, then one is longer than the other, and one can argue that a desired conclusion follows in 
either case. The other exception is that Euclid sometimes uses a reductio; for example, if the supposition that $a b$ and $c d$ are unequal yields a contradiction then one can conclude that $a b$ and $c d$ are equal. In our formal system, such case splits are always case splits on the truth of an atomic formula, and a proof by contradiction always establishes an atomic formula or its negation.

There is one more feature of Euclid's proofs that is worth calling attention to, namely, that in Euclid's proofs the construction steps generally precede the deductive conclusions. Thus, the proofs generally split into two phases: in the construction (kataskeue) phase, one carries out the construction, introducing all the objects that will be needed to reach the desired conclusion; and then in the deduction (apodeixis) phase one infers metric and diagrammatic consequences (see [36, pp. 159-160] or [16, vol. I, pp. 129-130]). This division is not required by our formal system, which is to say, nothing goes wrong in our proof system if one constructs some objects, draws some conclusions, and then carries out another construction. In other words, we take the division into the two phases to be a stylistic choice, rather than a logical necessity. For the most part, one can follow this stylistic prescription within $E$, and carry out all the constructions first. An exception to this occurs when, by $E$ 's lights, some deductive reasoning is required to ensure that prerequisites for carrying out a construction step are met. For example, we will see in Section 4.3 that our formal system takes issue with Euclid's proof of Proposition I.2: where Euclid carries out a complex construction without further justification, our system requires an explicit (but brief) argument, amidst the construction, to ensure that a certain point lies inside a certain circle. But even Euclid himself sometimes fails to maintain the division between the two phases, and includes demonstrative arguments in

the construction phase; see, for example, our discussion of Euclid's proof of Proposition I.44, in Section 4.3 Thus, our interpretation of the usual division of a Euclidean proof into construction and deduction phases is supported by the text of the Elements itself.

\subsection{Nondegeneracy assumptions}

As illustrated by our examples, Euclid typically assumes his geometric configurations are nondegenerate. For example, if $a$ and $b$ are given as arbitrary points, Euclid assumes they are distinct points, and if $a b c$ is a triangle, the points $a, b$, and $c$ are further assumed to be noncollinear. These are also sometimes called "genericity assumptions"; we are following Wu [65] in using the term "nondegeneracy."

Insofar as these assumptions are implicit in Euclid, his presentation can be criticized on two grounds:

1. The theorems are not always as strong as they can be, because the conclusions sometimes can still be shown to hold when some of the nondegeneracy constraints are relaxed. (Sometimes one needs to clarify the reading of the conclusion in a degenerate case.)

2. There are inferential gaps: when Euclid applies a theorem to the diagram 
obtained from a construction in the proof of a later theorem, he does not check that the nondegeneracy assumptions hold, or can be assumed to hold without loss of generality.

With respect the second criticism, Wu writes:

In the proof of a theorem, even though the configuration of the hypothesis at the outset is located in a generic, nondegenerate position, we are still unable to determine ahead of time whether or not the degenerate cases will occur when applying other theorems in the proof process. Not only is the verification of every applied theorem cumbersome and difficult, but it is actually also impossible to guarantee that the degenerate cases (in which the theorem is meaningless or false) do not happen in the proof process. On the other hand, we have no effective means to judge how much to restrict the statement of a theorem (to be proved) in order to ensure the truth of the theorem. These problems make it impossible for the Euclidean method of theorem proving to meet the requirements of necessary rigor. 65. p. 118]

Wu's comments refer to geometric theorem proving in general, not just the theorems of the Elements. With respect to the latter, we feel that the quote overstates the case: for the most part, the nondegeneracy requirements for theorem application in Euclid are easily met by assuming that the construction is appropriately generic. We discuss a mild exception in Section 4.3 noting that, according to $E$, Euclid should have said a few more words in the proof of Proposition I.9. But we do not know of any examples where substantial changes are needed.

Furthermore, the first criticism is only damning insofar as the degenerate cases are genuinely interesting. Nonetheless, from a modern standpoint, it is better to articulate just what is required in the statement of a theorem. Thus, we have chosen to "go modern" with $E$, in the sense that any distinctness assumptions (inequality of points, non-incidence of points and lines) that are required have to be stated explicitly as hypotheses. Although this marks a slight departure from Euclid, the fact that all assumptions are made explicit provides a more flexible framework to explore the issue as to which assumptions are implicit in his proofs.

\subsection{Our methodology}

We have cast our project as an attempt to model Euclidean diagrammatic proof, aiming to clarify its logical form, and, in particular, the nature of diagrammatic inference. In casting our project in this way, we are adopting a certain methodological stance. From a logical standpoint, what makes a Euclidean proof "diagrammatic" is not the fact that we find it helpful to consult a diagram in order to verify the correctness of the proof, or that, in the absence of such a physical artifact, we tend to roll our eyes towards the back of our heads and imagine 
such a diagram. Rather, the salient feature of Euclidean proof is that certain sorts of inferences are admitted as basic, and are made without further justification. When we say we are analyzing Euclidean diagrammatic reasoning, we mean simply that we are trying to determine which inferences have this basic character, in contrast to the geometrically valid inferences that are spelled out in greater detail in the text of the Elements.

Our analysis may therefore seem somewhat unsatisfying, in the sense that we do not attempt to explain why the fundamental methods of inference in the Elements are, or can be, or should be, taken to be basic. This is not to imply that we do not take such questions to be important. Indeed, it is just because they are such obvious and important questions that we are taking pains to emphasize the restricted character of our project.

What makes these questions difficult is that it is often not clear just what type of answer or explanation one would like. In order to explain why Euclidean practice is the way it is, one might reasonably invoke historical, pedagogical, or more broadly philosophical considerations. It may therefore help to highlight various types of analysis that are not subsumed by our logical approach. It does not include, per se, any of the following:

- a historical analysis of how the Elements came to be and attained the features we have described;

- a philosophical analysis as to what characterizes the inferences above as epistemically special (beyond that they interpret the ruler-and-compass constructions of modern geometric formalizations, and are sound and complete for the corresponding semantics), or in what sense they should be accepted as "immediate";

- a psychological or cognitive or pedagogical analysis of the human abilities that make it possible, and useful, to understand proofs in that form; or

- a computational analysis as to the most efficient data structures and algorithms for verifying the inferences we have characterized as "Euclidean," complexity upper and lower bounds, or effective search procedures.

We do, however, take it to be an important methodological point that the questions we address here can be separated from these related issues. We hope, moreover, that the understanding of Euclidean proof that our analysis provides can support these other lines of inquiry. We return to a discussion of these issues in Section 7.

\section{The formal system $E$}

\subsection{The language of $E$}

The language of $E$ is six-sorted, with sorts for points, lines, circles, segments, angles, and areas. There are variables ranging over the first three sorts; we use 
variables $a, b, c, \ldots$ to range over points, $L, M, N, \ldots$ to range over lines, and $\alpha, \beta, \gamma, \ldots$ to range over circles. In addition to the equality symbol, we have the following basic relations on elements of these sorts:

- on $(a, L)$ : point $a$ is on line $L$

- same-side $(a, b, L)$ : points $a$ and $b$ are on the same side of line $L$

- between $(a, b, c)$ : points $a, b$, and $c$ are distinct and collinear, and $b$ is between $a$ and $c$

- on $(a, \alpha)$ : point $a$ is on circle $\alpha$

- inside $(a, \alpha)$ : point $a$ is inside circle $\alpha$

- $\operatorname{center}(a, \alpha)$ : point $a$ is the center of circle $\alpha$

Note that between $(a, b, c)$ denotes a strict betweenness relation, and same-side $(a, b, L)$ entails that neither $a$ nor $b$ is on $L$. We also have three versions of an additional relation symbol, to keep track of the intersection of lines and circles:

- intersects $(L, M)$ : line $L$ and $M$ intersect

- intersects $(L, \alpha)$ : line $L$ intersects circle $\alpha$

- intersects $(\alpha, \beta)$ : circles $\alpha$ and $\beta$ intersect

In each case, by "intersects" we really mean "intersects transversally." In other words, two lines intersect when they have exactly one point in common, and two lines, or a line and a circle, intersect when they have exactly two points in common.

The objects of the last three sorts represent magnitudes. There are no variables ranging over these sorts; instead, one obtains objects of these sorts by applying the following functions to points:

- segment $(a, b)$ : the length of the line segment from $a$ to $b$, written $\overline{a b}$

- angle $(a, b, c)$ : the magnitude of the angle $a b c$, written $\angle a b c$

- area $(a, b, c)$ : the area of triangle $a b c$, written $\triangle a b c$

In addition to the equality relation, we have an addition function, + , a lessthan relation, $<$, and a constant, 0 , on each magnitude sort. Thus, for example, the expression $\overline{a b}=\overline{c d}$ denotes that the line segment determined by $a$ and $b$ is congruent to the line segment determined by $c$ and $d$, and $\overline{a b}<\overline{c d}$ denotes that it is strictly shorter. The symbol 0 is included for convenience; we could have, in a manner more faithful to Euclid, taken magnitudes to be strictly positive, with only minor modifications to the axioms and rules of inference described below. Finally, we also include a constant, "right-angle," of the angle sort. Thus we model the statement " $a b c$ is a right angle" as $\angle a b c=$ right-angle. 
The assertion "between $(a, b, c)$ " is intended to denote that $b$ is strictly between $a$ and $c$, which is to say, it implies that $b$ is not equal to either $a$ or c. In Section 5, we will see that, in this respect, it differs from the primitive used by Tarski in his axiomatization of Euclidean geometry. One reason that we have chosen the strict version is that it seems more faithful to Euclidean practice; see the discussion in Section 2.5. Another is that it seems to have better computational properties; see Section 6 .

The atomic formulas are defined as usual. A literal is an atomic formula or a negated atomic formula. We will sometimes refer to literals as "assertions," since, as we have noted, statements found in proofs in the Elements are generally of this form (or, at most, conjunctions of such basic assertions). Literals involving the relations on the first three sorts are "diagrammatic assertions," and literals involving the relations on the last three sorts are "metric assertions."

Additional predicates can be defined in terms of the basic ones presented here. For example, we can take the assertion $\overline{a b} \leq \overline{c d}$ to be shorthand for $\neg(\overline{c d}<\overline{a b})$. Similarly, we can assert that $a$ and $b$ are on different sides of a line $L$, written diff-side $(a, b, L)$, by making the sequence of assertions $\neg$ on $(a, L), \neg$ on $(b, L), \neg$ same-side $(a, b, L)$. Similarly, we can define outside $(a, \alpha)$ to be the conjunction $\neg$ inside $(a, \alpha), \neg$ on $(a, \alpha)$. Definitional extensions like these are discussed in Section 4.1 .

It is worth mentioning, at this point, that diagrammatic assertions like ours rarely appear in the text of Euclid's proofs. Rather, they are implicitly the result of diagrammatic hypotheses and construction steps, and they, in turn, license further construction steps and deductive inferences. But this fact is adequately captured by $E$ : even though raw diagrammatic assertions may appear in proofs, the rules are designed so that typically they do not have to. Consider, for example, the example in Section 2.3. In our system, the construction "let $e$ be the point of intersection of $L$ and $M$ " is licensed by the diagrammatic assertion intersects $(L, M)$, which, in turn, is licensed by the fact that $M$ contains two points, $c$ and $d$, that are on opposite sides of $L$. But we will take the assertion intersects $(L, M)$ to be a direct consequence of diagrammatic assertions that result from the construction, which allows this fact to license the construction step without explicit mention. And once $e$ has been designated the point of intersection, the fact that $e$ is between $c$ and $d$ is another direct consequence of the diagram assertions in play, and hence can be used to license future constructions and metric assertions. We discuss the relationship between our formal language and the informal text of the Elements in more detail in Section 4.1

\subsection{Proofs in $E$}

Theorems in $E$ have the following logical form:

$$
\forall \vec{a}, \vec{L}, \vec{\alpha}(\varphi(\vec{a}, \vec{L}, \vec{\alpha}) \rightarrow \exists \vec{b}, \vec{M}, \vec{\beta} \psi(\vec{a}, \vec{b}, \vec{L}, \vec{M}, \vec{\alpha}, \vec{\beta})),
$$

where $\varphi$ is a conjunction of literals, and $\psi$ is either a conjunction of literals or the symbol $\perp$, for "falsity" or "contradiction." Put in words, theorems in $E$ 
make statements of the following sort:

Given a diagram consisting of some points, $\vec{a}$, some lines, $\vec{L}$, and some circles, $\vec{\alpha}$, satisfying assertions $\varphi$, one can construct points $\vec{b}$, lines $\vec{M}$, and circles $\vec{\beta}$, such that the resulting diagram satisfies assertions $\psi$.

If the list $\vec{b}, \vec{M}, \vec{\beta}$ is nonempty, the theorem is a "problem," in Proclus' terminology. If that list is empty and $\psi$ is not $\perp$, we have a "theorem," in Proclus' sense. If $\psi$ is $\perp$, the theorem asserts the impossibility of the configuration described by $\varphi$.

In our proof system, we will represent a conjunction of literals by the corresponding set of literals, and the initial universal quantifiers will be left implicit. Thus, theorems in our system will be modeled as sequents of the form

$$
\Gamma \Rightarrow \exists \vec{b}, \vec{M}, \vec{\beta} . \Delta,
$$

where $\Gamma$ and $\Delta$ are sets of literals, and $\vec{b}, \vec{M}, \vec{\beta}$ do not occur in $\Gamma$. Assuming the remaining variables in $\Gamma$ and $\Delta$ are among $\vec{a}, \vec{L}, \vec{\alpha}$, the interpretation of the sequent is as above: given objects $\vec{a}, \vec{L}, \vec{\alpha}$ satisfying the assertions in $\Gamma$, there are objects $\vec{b}, \vec{M}, \vec{\beta}$ satisfying the assertions in $\Delta$.

As is common in the proof theory literature, if $\Gamma$ and $\Gamma^{\prime}$ are finite sets of literals and $\varphi$ is a literal, we will use $\Gamma, \Gamma^{\prime}$ to abbreviate $\Gamma \cup \Gamma^{\prime}$ and $\Gamma, \varphi$ to abbreviate $\Gamma \cup\{\varphi\}$. Beware, though: in the literature it is more common to read sets on the right side of a sequent arrow disjunctively, rather than conjunctively, as we do. Thus the sequent above corresponds to the single-succedent sequent $\Gamma \Rightarrow \exists \vec{b}, \vec{M}, \vec{\beta}(\bigwedge \Delta)$ in a standard Gentzen calculus for first-order logic.

Having described the theorems in our system, we now describe the proofs. As noted in Section 2.4, there are two sorts of steps in a Euclidean proof: construction steps, which introduce new objects into the diagram, and deduction steps, which infer facts about objects that have already been introduced. Thus, after setting forth the hypotheses, a typical Euclidean proof might have the following form:

Let $a$ be a point such that ...

Let $b$ be a point such that...

Let $L$ be a line such that ...

.

Hence ...

Hence ...

Hence ...

Application of a previously proved theorem fits into this framework: if the theorem is a "problem," in Proclus' terminology, applying it is a construction step, while if it is a "theorem," applying it is a demonstration step. The linear format is occasionally broken by a proof by cases or a proof by contradiction, which temporarily introduces a new assumption. For example, a proof by cases might have the following form: 


\section{Suppose $A$.}

Hence ...

Hence ...

Hence $B$.

On the other hand, suppose not $A$.

Hence ...

Hence ...

Hence $B$.

Hence $B$.

Proofs in $E$ can be represented as sequences of assertions in this way, where the validity of the assertion given at any line in the proof depends on the hypotheses of the theorem, as well as any temporary assumptions that are in play. Below, however, we will adopt conventional proof-theoretic notation, and take each line of the proof to be a sequent $\Gamma \Rightarrow \exists \vec{x}$. $\Delta$, where $\Gamma$ represents all the assumptions that are operant at that stage of the proof, $\vec{x}$ represent all the geometric objects that have been introduced, and $\Delta$ represents all the conclusions that have been drawn.

Thus, in our formal presentation of the proof system, a single construction step involves passing from a sequent of the form $\Gamma \Rightarrow \exists \vec{x}$. $\Delta$ to a sequent of the form $\Gamma \Rightarrow \exists \vec{x}, \vec{y} . \Delta, \Delta^{\prime}$, where $\vec{y}$ are variables for points, lines, and/or circles that do not occur in the original sequent. That is, the step asserts the existence of the new objects, $\vec{y}$, with the properties asserted by $\Delta^{\prime}$. In contrast, demonstration steps pass from a sequent of the form $\Gamma \Rightarrow \exists \vec{x} . \Delta$ to one of the form $\Gamma \Rightarrow \exists \vec{x} . \Delta, \Delta^{\prime}$, without introducing new objects. These include:

- Diagrammatic inferences: here $\Delta^{\prime}$ consists of a direct diagrammatic consequence of diagrammatic assertions in $\Gamma, \Delta$;

- Metric inferences: here $\Delta^{\prime}$ consists of a direct metric consequence of metric assertions in $\Gamma, \Delta$; and

- Transfer inferences: here $\Delta^{\prime}$ consists of a metric or diagrammatic assertion that can be inferred from metric and diagrammatic diagrammatic assertions in $\Gamma, \Delta$.

We will describe these inferences in detail in the sections that follow.

We have already noted that applying a previously proved theorem may or may not introduce new objects. Suppose we have proved a theorem of the form $\Pi \Rightarrow \exists \vec{y}$. $\Theta$, and we are at a stage in our proof where we have established the sequent $\Gamma \Rightarrow \exists \vec{x} . \Delta$. The first theorem, that is, the hypotheses in $\Pi$, may concern a right triangle $a b c$, whereas we may wish to apply it to a right triangle def. Thus, the inference may require renaming the variables of the first theorem. Furthermore, we may wish to extract only some of the conclusions of the theorem, and discard the others. Applying such a theorem, formally, involves doing the following: 
- renaming the variables of $\Pi \Rightarrow \exists \vec{y}$. $\Theta$ to obtain a sequent $\Pi^{\prime} \Rightarrow \exists \vec{y}^{\prime}$. $\Theta^{\prime}$, so that all the free variables of that sequent are among the variables of $\Gamma, \Delta$, and the variables $\vec{y}^{\prime}$ do not occur in $\Gamma, \Delta$;

- checking that every element of $\Pi^{\prime}$ is a direct diagram or metric consequence of $\Gamma, \Delta$;

- selecting some subset $\Delta^{\prime}$ of the conclusions $\Theta^{\prime}$ and the sublist $\vec{z}$ of variables from among $\vec{y}^{\prime}$ that occur in $\Theta^{\prime}$;

- and then concluding the sequent $\Gamma \Rightarrow \exists \vec{x}, \vec{z} . \Delta, \Delta^{\prime}$.

In words, suppose that, assuming that some geometric objects satisfy the assertions $\Gamma$, we have constructed objects $\vec{x}$ satisfying $\Delta$. Suppose, further, that, by a previous theorem, the assertions in $\Gamma$ and $\Delta$ imply the existence of new objects $\vec{z}$ satisfying $\Delta^{\prime}$. Then we can introduce new objects $\vec{z}$, satisfying the assertions in $\Delta^{\prime}$.

We also adhere to the common proof-theoretic practice of representing our proofs as trees rather than sequences, where the sequent at each node is inferred from sequents at the node's immediate predecessors. For the most part, trees will be linear, in the sense that each node has a single predecessor. The only exceptions arise in a proof by cases or a proof by contradiction. In the first case, one can establish a conclusion using a case split on atomic formulas. Such a proof has the following form:

$$
\frac{\Gamma \Rightarrow \exists \vec{x} . \Delta \quad \Gamma, \Delta, \varphi \Rightarrow \exists \vec{y} . \Delta^{\prime} \quad \Gamma, \Delta, \neg \varphi \Rightarrow \exists \vec{y} . \Delta^{\prime}}{\Gamma \Rightarrow \exists \vec{x}, \vec{y} . \Delta, \Delta^{\prime}}
$$

In words, suppose that, given geometric objects satisfying the assertions $\Gamma$, we have constructed objects $\vec{x}$ satisfying $\Delta$. Suppose, further, that given objects satisfying $\Gamma$ and $\Delta$, we can construct additional objects $\vec{y}$ satisfying $\Delta^{\prime}$, whether or not $\varphi$ holds. Then, given geometric objects satisfying the assertions $\Gamma$, we can obtain objects $\vec{x}, \vec{y}$ satisfying the assertions in $\Delta, \Delta^{\prime}$.

Recall that we have included the symbol $\perp$, or "contradiction," among our basic atomic assertions. Since the rules described below allow one to infer anything from a contradiction, we can use case splits to simulate proof by contradiction, as follows. Suppose, assuming $\neg \varphi$, we establish $\perp$. Then from $\neg \varphi$ we can establish $\varphi$. Since $\varphi$ certainly follows from $\varphi$, we have shown that $\varphi$ follows in any case.

Finally, we need to model two "superposition" inferences used by Euclid in Propositions 4 and 8 of Book I, to establish the familiar "side-side-side" and "side-angle-side" criteria for triangle congruences. The interpretation of these rules has been an ongoing topic of discussion for Euclid's commentators (see Heath [16, pp.224-228,249-250], Mancosu [30, pp. 28-33], or Mueller [37, pp. 21-24]). But the inferences have a very natural modeling in our system, described in Section 3.7 below.

A proof that ends with the sequent $\Gamma \Rightarrow \exists \vec{x}^{\prime} . \Delta^{\prime}$ constitutes a proof of $\Gamma \Rightarrow \exists \vec{x} . \Delta$ exactly when there is a map $f$ from $\vec{x}$ to the variables of $\Gamma, \Delta^{\prime}$ such 
that, under the renaming, every element of $\Delta^{f}$ is contained in or a diagrammatic consequence of $\Delta^{\prime}$. In other words, we have succeeded in proving the theorem when we have constructed the requisite objects and shown that they have the claimed properties 3

We claim that our formal system captures all the essential features of the proofs found in Books I to IV of the Elements. To be more precise, the Elements includes a number of more complicated inferences that are easily modeled in terms of our basic rules. To start with, Euclid often uses more elaborate case splits than the ones defined above, for example, depending on whether one segment is shorter than, the same length as, or longer than another. This is easily represented in our system as a sequence of two case splits. Also, Euclid often implicitly restricts attention to one case, without loss of generality, where the treatment of the other is entirely symmetric. Furthermore, we have focused on triangles; the handling of convex figures like rectangles and their areas can be reduced to these by introducing defined predicates. In Section 4.1 we describe some of the ways that "syntactic sugar" could be used to make E's proofs even more like Euclid's. Thus a more precise formulation of our claim is that if we use a suitable textual representation of proofs, then, modulo syntactic conventions like these, proofs in our formal system look very much like the informal proofs found in the Elements 4 Some examples are presented in Section 4.2 below to help substantiate this claim. Some ways in which proofs in our system depart substantially from the text of the Elements are discussed in Section 4.3 ,

To complete our description of $E$, we now need to describe:

1. the construction rules,

2. the diagrammatic inferences,

3. the metric inferences,

4. the diagram-metric transfer inferences, and

5. the two superposition inferences.

These are presented in Sections 3.3 3.7. The diagrammatic inferences, metric inferences, and diagram-metric transfer inferences will be presented as lists of

\footnotetext{
${ }^{3}$ Note that the function $f$ can map an existentially quantified variable in $\vec{x}$ to one of the variables in $\Gamma$. This means that the theorem "assuming $p$ is on $L$, there is a point $q$ on $L$ " has the trivial proof: "assuming $p$ is on $L, p$ is on $L . "$

We are, however, glossing over some technical details concerning the usual treatment of bound variables and quantifiers. For example, technically, we should require that no variable of $\Gamma$ conflict with the bound variables $\vec{x}$ of the sequent. It may be convenient to assume that we simply use separate stocks of variables for free (implicitly universally quantified) variables and bound (existentially quantified) variables. Or, better, one should construe all our claims as holding "up to renaming of bound variables."

${ }^{4}$ The manner of presenting proofs used above, whereby suppositional reasoning is indicated by indenting or otherwise setting off subarguments, amounts to the use of what are known as "Fitch diagrams."

Since the objects constructed to satisfy the conclusion of a proof by cases can depend on the case, we have glossed over details as to how our formal case splits should be represented in Fitch-style proofs. But see the second example in Section 4.5 for one way of doing this.
} 
first-order axioms, whereas what we really mean is that in a proof one is allowed to introduce any "direct consequence" of those axioms. This requires us to spell out a notion of "direct consequence," which we do in Section 3.8. In the meanwhile, little harm will come of thinking of the direct consequences as being the assertions that are first order consequences of the axioms, together with the assertions in $\Gamma, \Delta$.

\subsection{Construction rules}

In this section, we present a list of construction rules for $E$. Formally, these are described by sequents of the form $\Pi \Rightarrow \exists \vec{x}$. $\Theta$, where the variables $\vec{x}$ do not appear in $\Pi$. Applying such a construction rule means simply applying this sequent as a theorem, as described in the last section. In other words, one can view our construction rules as a list of "built-in" theorems that are available from the start. Intuitively, $\vec{x}$ are the objects that are constructed by the rule; $\Pi$ are the preconditions that guarantee that the construction is possible 5 and $\Theta$ are the properties that characterize the objects that are constructed.

We pause to comment on our terminology. What the rules below have in common is that they serve to introduce new objects to the diagram. Sometimes an object that is introduced is uniquely determined, as is the case, for example, with the rule "let $a$ be the intersection of $L$ and $M$." In other cases, there is an arbitrary choice involved, as is the case with the rule "let $a$ be a point on $L "$. We are referring to both as "construction rules," though one might object that picking a point is not really a "construction." It might be more accurate to describe them as "rules that introduce new objects into the diagram," but we have opted for the shorter locution. Our choice is made reasonable by the fact that the rules are all components of Euclidean constructions. Insofar as picking a point $c$ and connecting it to two points $a$ and $b$ can be seen as "constructing a triangle on the segment $a b$," it is reasonable to call the rule that allows one to pick $c$ a "construction rule."

For readability, the sequents are described informally. First, we provide a natural-language description of the construction, such as "let $a$ be a point on $L$." This is followed by a more precise specification of the prerequisites to the construction (corresponding to $\Pi$ in the sequent $\Pi \Rightarrow \exists \vec{x}$. $\Theta$ ), and the conclusion (corresponding to $\Theta$ ). Furthermore, when one constructs a point on a line, for example, one has the freedom to choose such a point distinct from any of the other points already in the diagram, and to specify that it does not lie on various lines and circles. The ability to do so is indicated by the optional "[distinct from ...]" clause; for example, assuming the lines $L$ and $M$ do not coincide, one can say "let $a$ be a point on $L$, distinct from $b, M$, and $\alpha$." As noted in Section 2.5. both the ability to specify, and the requirement of specifying, such "distinctness" conditions marks a departure from Euclid. In the presentation of the construction rules below, such conditions are abbreviated "[distinct from

\footnotetext{
${ }^{5}$ The conditions that are prerequisite to a construction are called the diarismos by Proclus; see [16. Book I, p. 130] or [36] p. 160].
} 
...]." Similarly, the requirement that $L$ be distinct from all the other lines mentioned is abbreviated "[ $L$ is distinct from lines ... $]$," and so on. So the example we just considered is an instance of the second rule on the list that follows, and becomes

$$
L \neq M \Rightarrow \exists a . \text { on }(a, L), a \neq b, \neg \mathrm{on}(a, M), \neg \mathrm{on}(a, \alpha)
$$

when expressed in sequent form.

\section{Points}

1. Let $a$ be a point [distinct from ...].

Prerequisites: none

Conclusion: $[a$ is distinct from... $]$

2. Let $a$ be a point on $L$ [distinct from ...].

Prerequisites: $[L$ is distinct from lines...]

Conclusion: $a$ is on $L,[a$ is distinct from... $]$

3. Let $a$ be a point on $L$ between $b$ and $c$ [distinct from ...] .

Prerequisites: $b$ is on $L, c$ is on $L, b \neq c,[L$ is distinct from lines ...]

Conclusion: $a$ is on $L, a$ is between $b$ and $c$, [a is distinct from...]

4. Let $a$ be a point on $L$ extending the segment from $b$ to $c$ [with $a$ distinct from...].

Prerequisites: $b$ is on $L, c$ is on $L, b \neq c,[L$ is distinct from lines ... ]

Conclusion: $a$ is on $L, c$ is between $b$ and $a,[a$ is distinct from...]

5. Let $a$ be a point on the same side of $L$ as $b$ [distinct from...]

Prerequisite: $b$ is not on $L$

Conclusion: $a$ is on the same side of $L$ as $b,[a$ is distinct from... $]$

6. Let $a$ be a point on the side of $L$ opposite $b$ [distinct from...]

Prerequisite: $b$ is not on $L$.

Conclusion: $a$ is not on $L, a$ is on the same side of $L$ as $b,[a$ is distinct from...]

7. Let $a$ be a point on $\alpha$ [distinct from ...].

Prerequisite: $[\alpha$ is distinct from other circles $]$

Conclusion: $a$ is on $\alpha,[a$ is distinct from...]

8. Let $a$ be a point inside $\alpha$ [distinct from .... .

Prerequisites: none

Conclusion: $a$ is inside $\alpha,[a$ is distinct from... $]$

9. Let $a$ be a point outside $\alpha$ [distinct from .... .

Prerequisites: none

Conclusion: $a$ is outside $\alpha,[a$ is distinct from... $]$ 


\section{Lines and circles}

1. Let $L$ be the line through $a$ and $b$.

Prerequisite: $a \neq b$

Conclusion: $a$ is on $L, b$ is on $L$

2. Let $\alpha$ be the circle with center $a$ passing through $b$.

Prerequisite: $a \neq b$

Conclusion: $a$ is the center of $\alpha, b$ is on $\alpha$

To make sense of the next list of constructions, recall that we are using the word "intersect" to refer to transversal intersection. For example, saying that two circles intersect means that they meet in exactly two distinct points.

\section{Intersections}

1. Let $a$ be the intersection of $L$ and $M$.

Prerequisite: $L$ and $M$ intersect

Conclusion: $a$ is on $L, a$ is on $M$

2. Let $a$ be a point of intersection of $\alpha$ and $L$.

Prerequisite: $\alpha$ and $L$ intersect

Conclusion: $a$ is on $\alpha, a$ is on $L$

3. Let $a$ and $b$ be the two points of intersection of $\alpha$ and $L$.

Prerequisite: $\alpha$ and $L$ intersect

Conclusion: $a$ is on $\alpha, a$ is on $L, b$ is on $\alpha, b$ is on $L, a \neq b$

4. Let $a$ be the point of intersection of $L$ and $\alpha$ between $b$ and $c$.

Prerequisites: $b$ is inside $\alpha, b$ is on $L, c$ is not inside $\alpha, c$ is not on $\alpha, c$ is on $L$

Conclusion: $a$ is on $\alpha, a$ is on $L, a$ is between $b$ and $c$

5. Let $a$ be the point of intersection of $L$ and $\alpha$ extending the segment from $c$ to $b$.

Prerequisites: $b$ is inside $\alpha, b$ is on $L, c \neq b, c$ is on $L$.

Conclusion: $a$ is on $\alpha, a$ is on $L, b$ is between $a$ and $c$

6. Let $a$ be a point on the intersection of $\alpha$ and $\beta$.

Prerequisite: $\alpha$ and $\beta$ intersect

Conclusion: $a$ is on $\alpha, a$ is on $\beta$

7. Let $a$ and $b$ be the two points of intersection of $\alpha$ and $\beta$.

Prerequisite: $\alpha$ and $\beta$ intersect

Conclusion: $a$ is on $\alpha, a$ is on $\beta, b$ is on $\alpha, b$ is on $\beta, a \neq b$ 

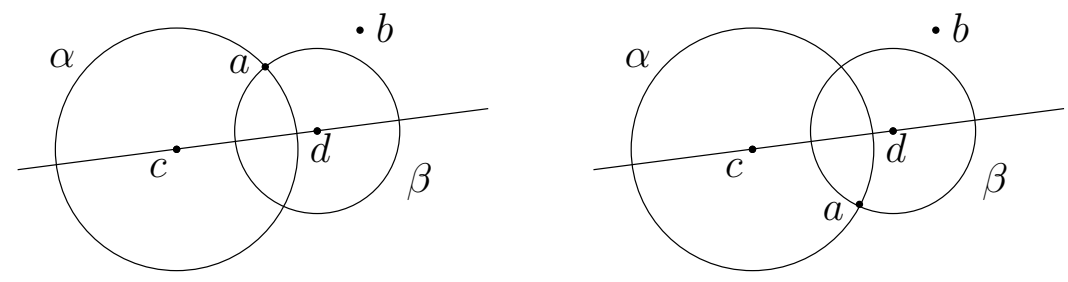

Figure 1: Diagrams for intersection rules 8 (left) and 9 (right). In the first, the added intersection point $a$ is on the same side of $L$ as $b$; in the second, it is opposite $b$.

8. Let $a$ be the point of intersection of $\alpha$ and $\beta$, on the same side of $L$ as $b$, where $L$ is the line through their centers, $c$ and $d$, respectively.

Prerequisites: $\alpha$ and $\beta$ intersect, $c$ is the center of $\alpha, d$ is the center of $\beta$, $c$ is on $L, d$ is on $L, b$ is not on $L$

Conclusion: $a$ is on $\alpha, a$ is on $\beta, a$ and $b$ are on the same side of $L$

9. Let $a$ be the point of intersection of $\alpha$ and $\beta$, on the side of $L$ opposite $b$, where $L$ is the line through their centers, $c$ and $d$, respectively.

Prerequisite: $\alpha$ and $\beta$ intersect, $c$ is the center of $\alpha, d$ is the center of $\beta$, $c$ is on $L, d$ is on $L, b$ is not on $L$

Conclusion: $a$ is on $\alpha, a$ is on $\beta, a$ and $b$ are not on the same side of $L, a$ is not on $L$.

We close this section by noting that there is some redundancy in our construction rules. For example, the circle intersection rules 8 and 9, which are somewhat complex, could be derived as theorems from the more basic rules. As we will see below, we have added them to model particular construction steps in the Elements. But there are other constructions that can be derived in our system, that seem no less obvious; for example, if $M$ and $N$ are distinct lines that intersect, and $a$ is not on $N$, then one can pick a point $b$ on $M$ on the same side of $N$ as $a$. We did not include this rule only because we did not find it in Euclid, though constructions like this come up in our completeness proof, in Section 5

This situation is somewhat unsatisfying. Our list of construction rules was designed with two goals in mind: first, to model the constructions in Euclid, and, second, to provide a system that is complete, in the sense described in Section 5. But a smaller set of rules would have met the second constraint, and since the constructions appearing in Books I to IV of the Elements constitute a finite list, the first constraint could be met by brute-force enumeration. What is missing is a principled determination of what should constitute an "obvious" construction, as opposed to an existence assertion that requires explicit proof.

We did, at one point, consider allowing the prover to introduce any point satisfying constraints that are consistent with the current diagram. Even for diagrams without circles, however, determining whether such a list of constraints 
meets this criterion seems to be a knotty combinatorial problem. And since circles can encode metric information, in that case the proposal seems to allow users to do things that are far from obvious. In any event, it is not clear that this proposal comes closer to characterizing what we should take as "obvious constructions." We therefore leave this task as an open conceptual problem, maintaining only that the list of constructions we have chosen here are (1) obviously sound, in an informal sense; (2) sufficient to emulate the proofs in Books I to IV of the Elements; (3) sound for the intended semantics; and (4) sufficient to make the system complete.

\subsection{Diagrammatic inferences}

We now provide a list of axioms that allow us to infer diagrammatic assertions from the diagrammatic information available in a given context in a proof. For the moment, these can be read as first-order axioms; the precise sense in which they can be used to license inferences in $E$ is spelled out in Section 3.8 .

\section{Generalities}

1. If $a \neq b, a$ is on $L$, and $b$ is on $L, a$ is on $M$ and $b$ is on $M$, then $L=M$.

2. If $a$ and $b$ are both centers of $\alpha$ then $a=b$.

3. If $a$ is the center of $\alpha$ then $a$ is inside $\alpha$.

4. If $a$ is inside $\alpha$, then $a$ is not on $\alpha$.

The first axiom above says that two points determine a line. It is logically equivalent to the assertion that the intersection of two distinct lines, $L$ and $M$, is unique. The next two axioms tell us that the center of a circle is unique, and inside the circle. The final axiom then rules out "degenerate" circles.

\section{Between axioms}

1. If $b$ is between $a$ and $c$ then $b$ is between $c$ and $a, a \neq b, a \neq c$, and $a$ is not between $b$ and $c$.

2. If $b$ is between $a$ and $c, a$ is on $L$, and $b$ is on $L$, then $c$ is on $L$.

3. If $b$ is between $a$ and $c, a$ is on $L$, and $c$ is on $L$, then $b$ is on $L$.

4. If $b$ is between $a$ and $c$ and $d$ is between $a$ and $b$ then $d$ is between $a$ and $c$.

5. If $b$ is between $a$ and $c$ and $c$ is between $b$ and $d$ then $b$ is between $a$ and $d$.

6. If $a, b$, and $c$ are distinct points on a line $L$, then then either $b$ is between $a$ and $c$, or $a$ is between $b$ and $c$, or $c$ is between $a$ and $b$. 
7. If $b$ is between $a$ and $c$ and $b$ is between $a$ and $d$ then $b$ is not between $c$ and $d$.

Axioms 1, 4, 5, and 6 are essentially the axioms for "between" given in Krantz et al. [12, with the minor difference that we are axiomatizing a "strict" notion of betweenness instead of a nonstrict one. Krantz et al. show that a countable set satisfies these axioms if and only if it can be embedded as a set of points on the real line. We remark, in passing, that it would be interesting to have similar completeness or representation theorems for other groups of the axioms found here. Our approach has been syntactic rather than semantic, which is to say, our goal has been to capture certain deductive relationships rather than to characterize classes of structures; but it would be illuminating to understand the extent to which our various groups of axioms give rise to natural classes of structures.

The last axiom is illustrated by the following diagram:

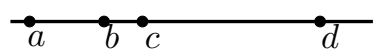

The axiom states that if $d$ and $c$ are on the same side of $b$ along a line, then $b$ does not fall between them. This axiom is, in fact, a first-order consequence of the others; it is therefore only useful in contexts where we consider more restrictive notions of consequence, as we do in Section 3.8.

\section{Same side axioms}

1. If $a$ is not on $L$, then $a$ and $a$ are on the same side of $L$.

2. If $a$ and $b$ are on the same side of $L$, then $b$ and $a$ are on the same side of $L$.

3. If $a$ and $b$ are on the same side of $L$, then $a$ is not on $L$.

4. If $a$ and $b$ are on the same side of $L$, and $a$ and $c$ are on the same side of $L$, then $b$ and $c$ are on the same side of $L$.

5. If $a, b$, and $c$ are not on $L$, and $a$ and $b$ are not on the same side of $L$, then either $a$ and $c$ are on the same side of $L$, or $b$ and $c$ are on the same side of $L$.

If $L$ is a line, the axioms imply that the relation "falling on the same side of $L$ " is an equivalence relation; and any point $a$ not on $L$ serves to partition the points into three classes, namely, those on $L$, those on the same side of $L$ as $a$, and those on the opposite side of $L$ from $a$.

With the interpretation of diff-side $(p, q, L)$ described in Section 3.1 the axioms imply that if $a$ and $b$ are on different sides of $L$ and $a$ and $c$ are on different sides of $L$, then $b$ and $c$ are on the same side of $L$. The axioms also imply that if $a$ and $b$ are on the same side of $L$ and $a$ and $c$ are on different sides of $L$ then $b$ and $c$ are on different sides of $L$.

\section{Pasch axioms}



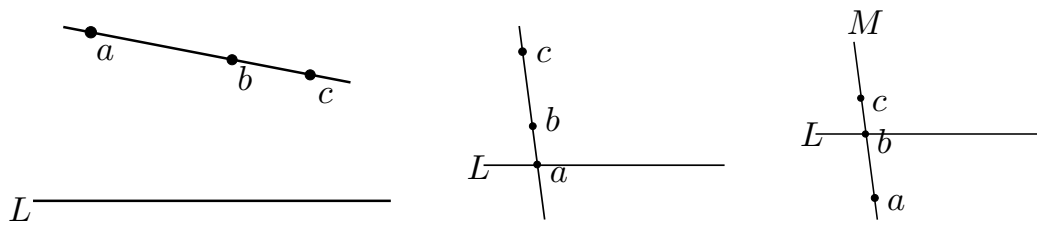

Figure 2: Pasch rules 1 (left), 2 (center), and 3 and 4 (right).

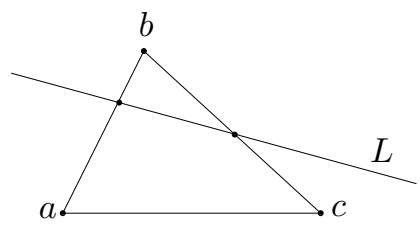

Figure 3: the Pasch axiom

1. If $b$ is between $a$ and $c$ and $a$ and $c$ are on the same side of $L$, then $a$ and $b$ are on the same side of $L$.

2. If $b$ is between $a$ and $c$ and $a$ is on $L$ and $b$ is not on $L$, then $b$ and $c$ are on the same side of $L$.

3. If $b$ is between $a$ and $c$ and $b$ is on $L$ then $a$ and $c$ are not on the same side of $L$.

4. If $b$ is the intersection of distinct lines $L$ and $M, a$ and $c$ are distinct points on $M, a \neq b, c \neq b$, and $a$ and $c$ are not on the same side of $L$, then $b$ is between $a$ and $c$.

These axioms serve to relate the "between" relation and the "same side" relation. In the fourth axiom, " $b$ is the intersection of distinct lines $L$ and $M$ " should be understood as " $L \neq M, b$ is on $L$, and $b$ is on $M . "$

In the literature, the phrase "Pasch axiom" is used to refer to the assertion that a line passing through one side of a triangle necessarily passes through one of the other two sides, or their point of intersection (see Figure 3). This axiom was indeed used by Pasch [46, and later by Hilbert [22, with attribution. Theorems of $E$ do not allow disjunctive conclusions, but one can use the conclusion of Pasch's theorem to reason disjunctively in a proof: in Figure 3, either $c$ is on $L$, or on the same side of $L$ as $a$, or on the same side of $L$ as $b$. In the second case, where $a$ and $c$ are on the same side of $L$, our third Pasch axiom (together with the same-side axioms) imply that $b$ and $c$ are on opposite sides of $L$. The intersection rules below then tell us that the line through $b$ and $c$ intersects $L$. Our fourth Pasch axiom then implies that this intersection is between $b$ and $c$. The third case is handled in a similar way. We have therefore chosen the name for this group of axioms to indicate that they provide an analysis of the usual Pasch axiom into more basic diagrammatic rules. 


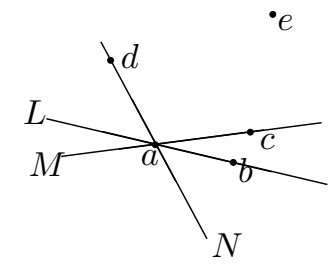

Figure 4: Triple incidence rules. (The same diagram illustrates all three rules.)

\section{Triple incidence axioms}

1. If $L, M$, and $N$ are lines meeting at a point $a$, and $b, c$, and $d$ are points on $L, M$, and $N$ respectively, and if $c$ and $d$ are on the same side of $L$, and $b$ and $c$ are on the same side of $N$, then $b$ and $d$ are not on the same side of $M$.

2. If $L, M$, and $N$ are lines meeting at a point $a$, and $b, c$, and $d$ are points on $L, M$, and $N$ respectively, and if $c$ and $d$ are on the same side of $L$, and $b$ and $d$ are not on the same side of $M$, and $d$ is not on $M$ and $b \neq a$, then $b$ and $c$ are on the same side of $N$.

3. If $L, M$, and $N$ are lines meeting at a point $a$, and $b, c$, and $d$ are points on $L, M$, and $N$ respectively, and if $c$ and $d$ are on the same side of $L$, and $b$ and $c$ are on the same side of $N$, and $d$ and $e$ are on the same side of $M$, and $c$ and $e$ are on the same side of $N$, then $c$ and $e$ are on the same side of $L$.

These axioms explain how three lines intersecting in a point divide space into regions (see diagram 3.4).

\section{Circle axioms}

1. If $a, b$, and $c$ are on $L, a$ is inside $\alpha, b$ and $c$ are on $\alpha$, and $b \neq c$, then $a$ is between $b$ and $c$.

2. If $a$ and $b$ are each inside $\alpha$ or on $\alpha$, and $c$ is between $a$ and $b$, then $c$ is inside $\alpha$.

3. If $a$ is inside $\alpha$ or on $\alpha, c$ is not inside $\alpha$, and $c$ is between $a$ and $b$, then $b$ is neither inside $\alpha$ nor on $\alpha$.

4. Let $\alpha$ and $\beta$ be distinct circles that intersect in distinct points $c$ and $d$. Let $a$ be a the center of $\alpha$, let $b$ be the center of $\beta$, and let $L$ be the line through $a$ and $b$. Then $c$ and $d$ are not on the same side of $L$.

\section{Intersection rules}




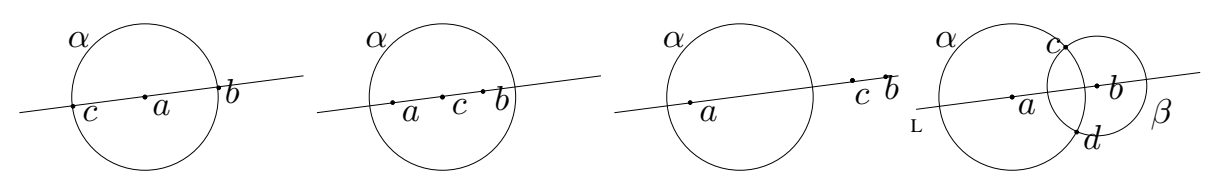

Figure 5: Circle axioms 1-4.

1. If $a$ and $b$ are on different sides of $L$, and $M$ is the line through $a$ and $b$, then $L$ and $M$ intersect.

2. If $a$ is on or inside $\alpha, b$ is on or inside $\alpha$, and $a$ and $b$ are on different sides of $L$, then $L$ and $\alpha$ intersect.

3. If $a$ is inside $\alpha$ and on $L$, then $L$ and $\alpha$ intersect.

4. If $a$ is on or inside $\alpha, b$ is on or inside $\alpha, a$ is inside $\beta$, and $b$ is outside $\beta$, then $\alpha$ and $\beta$ intersect.

5. If $a$ is on $\alpha, b$ is in $\alpha, a$ is in $\beta$, and $b$ is on $\beta$, then $\alpha$ and $\beta$ intersect.

Recall that "intersection" means transversal intersection. The first axiom says that a line passing from one side of $L$ to the other intersects it. The second axiom says that if $\alpha$ is a circle that straddles $L$, then $\alpha$ intersects $L$. The third axiom says that a line that passes through a circle intersects it. The fourth and fifth axioms are the analogous properties for circles. The third axiom can be viewed as the assertion that a line cannot be bounded by a circle; the others can be viewed as continuity principles.

\section{Equality axioms}

1. $x=x$

2. If $x=y$ and $\varphi(x)$, then $\varphi(y)$

Here $x$ and $y$ can range over any of the sorts (that is, there is an equality symbol for each sort) and $\varphi$ can be any atomic formula. These are the usual equality axioms for first-order logic, and so may be taken to be subsumed under the notion of "first-order consequence."

\subsection{Metric inferences}

Consider the structure $\left\langle\mathbb{R}^{+}, 0,+,<\right\rangle$, that is, the nonnegative real numbers with zero, addition, and the less-than relation. It is well known that the theory of this structure is decidable. The set of universal consequences of this theory (or, equivalently, the set of quantifier-free formulas that are true of the structure under any assignment to the free variables) can be axiomatized as follows:

- + is associative and commutative, with identity 0 . 
- $<$ is a linear ordering with least element 0 .

- For any $x, y$, and $z$, if $x<y$ then $x+z<y+z$.

Equivalently, these axioms describe the nonnegative part of any linearly ordered abelian group. Happily, these are the general properties Euclid assumes of magnitudes, that is, the segment lengths, angles, and areas in our formalization (see Stein [55, p. 167]). To be more precise, Euclid seems to assume that his magnitudes are strictly positive. But we have already noted in Section 3.1 that we simply include 0 for convenience; we could just as well have axiomatized the strictly positive reals. The axioms above imply that if $x+z=y+z$, then $x=z$, which corresponds to Euclid's common notion 3, "if equals be subtracted from equals, the remainders are equal." The third axiom implies that if $0<y$, then $z<y+z$, which corresponds to common notion 5, "the whole is greater than the part."

In addition to these, we include the following axioms, which Euclid seems to take to be clear from the definitions (modulo the caveat, in the last paragraph, that we include 0 as a magnitude):

1. $\overline{a b}=0$ if and only if $a=b$.

2. $\overline{a b} \geq 0$

3. $\overline{a b}=\overline{b a}$.

4. $a \neq b$ and $a \neq c$ imply $\angle a b c=\angle c b a$.

5. $0 \leq \angle a b c$ and $\angle a b c \leq$ right-angle + right-angle.

6. $\triangle a a b=0$.

7. $\triangle a b c \geq 0$.

8. $\triangle a b c=\triangle c a b$ and $\triangle a b c=\triangle a c b$.

9. If $\overline{a b}=\overline{a^{\prime} b^{\prime}}, \overline{b c}=\overline{b^{\prime} c^{\prime}}, \overline{c a}=\overline{c^{\prime} a^{\prime}}, \angle a b c=\angle a^{\prime} b^{\prime} c^{\prime}, \angle b c a=\angle b^{\prime} c^{\prime} a^{\prime}$, and $\angle c a b=\angle c^{\prime} a^{\prime} b^{\prime}$, then $\triangle a b c=\triangle a^{\prime} b^{\prime} c^{\prime}$.

Note that we do not ascribe any meaning to the magnitude $\angle a b c$ when $b=a$ or $b=c$. As axiom 6 indicates, however, we take "degenerate" triangles to have area 0. Once Euclid has proved two triangles congruent (that is, once he has shown that all their parts are equal), he uses the fact that they have the same area, without comment. The last axiom simply makes this explicit.

Of course, there are further properties involving magnitudes that can be read off from a diagram, and, conversely, metric considerations can imply diagrammatic facts. These "transfer inferences" are the subject of the next section. 


\subsection{Transfer inferences}

We divide the transfer inferences into three groups, depending on whether they involve segment lengths, angles, or areas.

\section{Diagram-segment transfer axioms}

1. If $b$ is between $a$ and $c$, then $\overline{a b}+\overline{b c}=\overline{a c}$.

2. If $a$ is the center of $\alpha$ and $\beta, b$ is on $\alpha, c$ is on $\beta$, and $\overline{a b}=\overline{a c}$, then $\alpha=\beta$.

3. If $a$ is the center of $\alpha$ and $b$ is on $\alpha$, then $\overline{a c}=\overline{a b}$ if and only if $c$ is on $\alpha$.

4. If $a$ is the center of $\alpha$ and $b$ is on $\alpha$, and $\overline{a c}<\overline{a b}$ if and only if $c$ is in $\alpha$.

The second axiom implies that a circle is determined by its center and radius. In the discussion in Section 4.3, we will explain that this is a mild departure from Euclid's treatment of circles. (Euclid seems to rely on a construction rule which has the same net effect.) When $\alpha=\beta$, this axiom implies the converse direction of the equivalence in axiom 3 (so that axiom could be stated instead as an implication).

\section{Diagram-angle transfer axioms}

1. Suppose $a \neq b, a \neq c, a$ is on $L$, and $b$ is on $L$. Then $c$ is on $L$ and $a$ is not between $b$ and $c$ if and only if $\angle b a c=0$.

2. Suppose $a$ is on $L$ and $M, b$ is on $L, c$ is on $M, a \neq q, a \neq c, d$ is not on $L$ or $M$, and $L \neq M$. Then $\angle b a c=\angle b a d+\angle d a c$ if and only if $b$ and $d$ are on the same side of $M$ and $c$ and $d$ are on the same side of $L$.

3. Suppose $a$ and $b$ are points on $L, c$ is between $a$ and $b$, and $d$ is not on $L$. Then $\angle a c d=\angle d c b$ if and only if $\angle a c d$ is equal to right-angle.

4. Suppose $a, b$, and $b^{\prime}$ are on $L, a, c$, and $c^{\prime}$ are on $M, b \neq a, b^{\prime} \neq a, c \neq a$, $c^{\prime} \neq a, a$ is not between $b$ and $b^{\prime}$, and $a$ is not between $c$ and $c^{\prime}$. Then $\angle b a c=\angle b^{\prime} a c^{\prime}$.

5. Suppose $a$ and $b$ are on $L, b$ and $c$ are on $M$, and $c$ and $d$ are on $N$. Suppose also that $b \neq c, a$ and $d$ are on the same side of $N$, and $\angle a b c+\angle b c d<$ right-angle + right-angle. Then $L$ and $N$ intersect, and if $e$ is on $L$ and $N$, then $e$ and $a$ are on the same side of $M$.

The first axiom says that if $a$ and $b$ are distinct points on a line $L$, then a point $c$ is on $L$ on the same side of $a$ as $b$ if and only if $\angle b a c=0$. The right-hand side of the equivalence in the second axiom can be read more simply as the assertion that $d$ lies inside the angle bac. Thus the axiom implies that angles sum in the expected way. The third axiom corresponds to Euclid's definition 10, "when a straight line set up on a straight line makes the adjacent angles equal to one another, each of the equal angles is called right...." It also, at the 


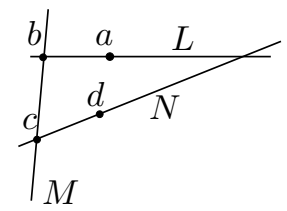

Figure 6: diagram-angle transfer axiom 5.

same time, codifies postulate 4, "all right angles are equal to one another," using the constant, "right-angle," to describe the magnitude that all right angles are equal to. The fourth axiom says that different descriptions of the same angle are equal; more precisely, if $a b$ and $a b^{\prime}$ are the same ray, and likewise for $a c$ and $a c^{\prime}$, then $a b c$ and $a b^{\prime} c$ are equal.

Euclid's wording may make it seem more natural to use a predicate to assert that $a b c$ forms a right angle, rather than using a constant, "right-angle," to denote an arbitrary right angle. But Euclid seems to refer to an arbitrary right angle in his statement of this parallel postulate, which, in the Heath translation, states:

That, if a straight line falling on two straight lines make the interior angles on the same side less than two right angles, the two straight lines, if produced indefinitely, meet on that side on which are the angles less than the two right angles. [16, p. 155]

Formulated in this way, a better name for the axiom might be the "non-parallel postulate": it asserts that if the diagram configuration satisfies the given metric constraints on the angles, then two of the lines are guaranteed to intersect. The postulate translates to the last axiom above, which licenses the construction "let $e$ be the intersection of $L$ and $N$." Furthermore, assuming $e$ is the intersection of $L$ and $N$, the postulate specifies the side of $M$ on which $e$ lies.

\section{Diagram-area transfer axioms}

1. If $a$ and $b$ are on $L$ and $a \neq b$, then $\triangle a b c=0$ if and only if $c$ is on $L$.

2. If $a, b, c$ are on $L$ and distinct from one another, $d$ is not on $L$, then $c$ is between $a$ and $b$ if and only if $\triangle a c d+\triangle d c b=\triangle a d b$.

The second axiom implies that when a triangle is divided in two, the areas sum in the expected way.

\subsection{Superposition}

We now come to the final two inferences in our system, Euclid's notorious "superposition inferences," which vexed commentators through the ages (see the references in Section 3.2). Euclid's Proposition I.4 states the familiar "sideangle-side" property, namely that if two triangles $a b c$ and def are such that 


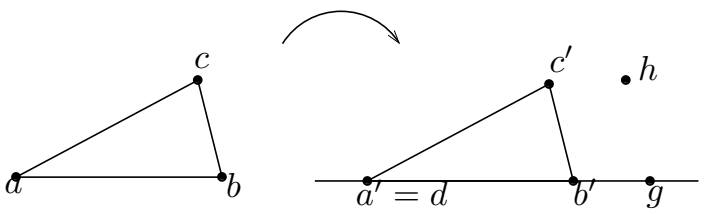

Figure 7: superposition

$a b, a c$ are congruent to $d e, d f$ respectively, and $b a c$ is congruent to angle $e d f$, then the two triangles are congruent. The proof proceeds by imagining $a b c$ superimposed on def. In the Heath translation:

For, if the triangle $a b c$ be applied to the triangle $\operatorname{def}$, and if the point $a$ be placed on the point $d$ and the straight line $a b$ on $d e$, then the point $b$ will also coincide with $e$, because $a b$ is equal to $d e \ldots$. 16, p. 247]

At issue is what it means to "apply" $a b c$ to another triangle in such a way. Euclid has not yet proved that one can construct a copy of $a^{\prime} b^{\prime} c^{\prime}$ of $a b c$ that will meet the given constraints. This requires one to be able to copy a given angle, which is Euclid's Proposition I.23. The chain of reasoning leading to that proposition includes Proposition I.4 as a component. The same issue arises in the proof of Proposition I.8, which uses a superposition argument to establish the "side-side-side" property.

How, then, shall we treat superposition? One possibility is simply to add two new construction rules. The first would assert that given an angle $a b c$, a point $d$ on a line $L$, a point $g$ on $L$, and a point $h$ not on $L$, one can construct points $a^{\prime}, b^{\prime}, c^{\prime}$ such that $a^{\prime}=d, \angle a^{\prime} b^{\prime} c^{\prime}=\angle a b c, b^{\prime}$ lies on $L$ in the direction determined by $g$, and $c^{\prime}$ lies on the same side of $L$ as $h$. The second says that given a triangle $a b c$, a point $d$ on a line $L$, a point $g$ on $L$, and a point $h$ not on $L$, once can find points $a^{\prime}, b^{\prime}, c^{\prime}$ as above with $a b, b c, c a$ congruent to $a^{\prime} b^{\prime}, b^{\prime} c^{\prime}, c^{\prime} a^{\prime}$, respectively. These new construction rules would certainly allow us to carry out the proofs of Propositions I.4 and I.8, but the solution is not at all satisfying: Euclid takes great pains to derive the fact that one can carry out constructions like these, using Propositions I.4 and I.8 along the way.

A second possibility is simply to add Propositions I.4 and I.8, the SAS and SSS properties, as axioms. But, once again, this is not a satisfactory solution, since it fails to explain why Euclid takes the trouble to prove them.

Our formulation of $E$ provides a third, more elegant solution. What superposition allows one to do is to act as though one has the result of doing the constructions above, but only for the sake of proving things about objects that are already present in the diagram. In proof-theoretic parlance, superposition is used as an elimination rule: if you can derive a conclusion assuming the existence of some new objects, you can infer that the conclusion holds without the additional assumption. In Euclid's case, one is barred, however, from using the assumption to construct new objects. 
This has a straightforward formulation as a sequent inference. Suppose $\Gamma, \Delta$ includes assertions to the effect that $a b c$ are distinct and noncollinear, and $g$, $L$, and $h$ are as above. Let $\Pi_{1}$ be the set

$$
\left\{a^{\prime}=d, \angle a^{\prime} b^{\prime} c^{\prime}=\angle a b c, \text { on }\left(b^{\prime}, L\right), \neg \operatorname{between}\left(b^{\prime}, d, g\right), \text { same-side }\left(c^{\prime}, h, L\right)\right\}
$$

corresponding to the result of SAS superposition, and let $\Pi_{2}$ be the set

$\left\{a^{\prime}=d, \overline{a b}=\overline{a^{\prime} b^{\prime}}, \overline{b c}=\overline{b^{\prime} c^{\prime}}, \overline{c a}=\overline{c^{\prime} a^{\prime}}\right.$, on $\left(b^{\prime}, L\right), \neg \operatorname{between}\left(b^{\prime}, d, g\right)$, same-side $\left.\left(c^{\prime}, h, L\right)\right\}$

corresponding to the results of SSS superposition. Then the rules can be expressed as

$$
\frac{\Gamma \Rightarrow \exists \vec{x} . \Delta \quad \Gamma, \Delta, \Pi_{i} \Rightarrow \Delta^{\prime}}{\Gamma \Rightarrow \exists \vec{x} . \Delta, \Delta^{\prime}}
$$

where $i$ is equal to 1,2 , respectively.

\subsection{The notion of a "direct consequence"}

We have characterized "the diagram" in a Euclidean proof as the collection of diagrammatic facts that have been established, either by construction or by inference, at a given point in the proof; and we have characterized the "diagrammatic inferences" as those diagrammatic facts that are "direct consequences" of those. The goal of this section is to complete the description of $E$ by spelling out an adequate notion of "direct consequence."

Our attempts to define such a notion are constrained by a number of desiderata. The first is fidelity to Euclid:

- The direct consequences of a set of diagrammatic hypotheses should provide an adequate model of the diagrammatic facts that Euclid makes use of in a proof, either explicitly or in licensing a construction or a metric conclusion, without explicit justification.

The next two are more mathematical:

- Any direct consequence should be, in particular, a first-order consequence of the diagrammatic axioms and diagrammatic facts in $\Gamma, \Delta$.

- Conversely, any diagrammatic assertion that is a first-order consequence of the diagrammatic axioms should be derivable in $E$, though not necessarily in one step.

The first constraint says that direct consequences of a set of diagrammatic assertions should be sound with respect to the set of first-order consequences of the diagrammatic axioms. The second constraint says that together with the other methods of proof provided by $E$, they should be complete as well. We will see that there is a lot of ground between these two constraints. For example, they can be met by taking the direct consequences to be all first-order 
consequences. But this overshoots our first desideratum, since it would let us make direct inferences that Euclid spells out more explicitly. Nor does it sit well with the notion of "directness." Since we are dealing with a universal theory in a language with no function symbols, the set of literals that are consequences of a given set $\Gamma$ of literals is decidable: one only need extract all instances of the axioms among the variables in $\Gamma$, and use a decision procedure for propositional logic. But this is unlikely to be computationally feasible 6 and we expect a "direct" inference to be more tame than that. Thus our third desiderata is of a computational nature:

- The problem of determining whether a literal is a direct consequence of some diagrammatic facts should be, in some sense, computationally tractable.

The notion of tractability should be taken with a grain of salt. It is loosely related to the practical question as to whether one can implement a proof checker for our formal system which performs reasonably on formalized proofs of statements in the Elements, a question we address in Section 6. But it is worth keeping in mind that even our theoretical characterization is only intended to be compelling at the level of complexity found in proofs in the Elements. When a diagram has millions of points, lines, and circles, we may be faulted for sanctioning "direct" inferences that cannot be carried out with our limited cognitive apparatus. But even propositional logic, as a model of logical inference, is subject to the same criticisms: can we really "recognize" an instance of modus ponens when the formulas involved have more than $2^{100}$ symbols?

To develop a notion of direct consequence, let us begin by noting that most of our axioms are naturally expressed as rules; in other words, they have the form

$$
\text { if } \varphi_{1}, \varphi_{2}, \ldots, \varphi_{n} \text { then } \psi
$$

where $\varphi_{1}, \ldots, \varphi_{n}, \psi$ are literals. The example in Section 2.2 suggests that we should be able to chain such rules; that is, whenever we know $\varphi_{1}, \ldots, \varphi_{n}$, we also know $\psi$, and can use $\psi$ to secure further knowledge. Occasionally, our diagrammatic axioms are not quite in rule form, with either a disjunction among the hypothesis or a conjunction in the conclusion. But this can be viewed as a notational convenience; the rule "if $\varphi_{1}, \varphi_{2}, \ldots, \varphi_{n}$ then $\psi$ and $\theta$ " is equivalent to the pair of rules "if $\varphi_{1}, \varphi_{2}, \ldots, \varphi_{n}$ then $\psi$ " and "if $\varphi_{1}, \varphi_{2}, \ldots, \varphi_{n}$ then $\theta$," and the rule "if $\varphi_{1}, \varphi_{2}, \ldots, \varphi_{n}$ and either $\theta$ or $\eta$ then $\psi$ " is equivalent to the pair of rules "if $\varphi_{1}, \varphi_{2}, \ldots, \varphi_{n}$ and $\theta$ then $\psi$ " and "if $\varphi_{1}, \varphi_{2}, \ldots, \varphi_{n}$ and $\eta$ then $\psi . "$

A moment's reflection, however, shows that we should also allow "contrapositive" variants of our rules. For example, consider the first Pasch axiom:

if $b$ is between $a$ and $c$ and $a$ and $c$ are on the same side of $L$, then $a$ and $b$ are on the same side of $L$

\footnotetext{
${ }^{6}$ We do not, however, have a lower bound on the computational complexity of the decision problem associated with our particular set of axioms.
} 
Certainly, if we know that $b$ is between $a$ and $c$ and that $a$ and $c$ are on the same side of $L$, we should be allowed to infer that $a$ and $b$ are on the same side of $L$. But suppose we know that $b$ is between $a$ and $c$ but that the conclusion fails, that is, $a$ and $b$ are not on the same side of $L$. Drawing a picture or imagining the situation in our mind's eye enables us to see, straightforwardly, that the second hypothesis fails, that is, $a$ and $c$ are not on the same side of $L$. In other words, we should include the rule

if $b$ is between $a$ and $c$ and $a$ and $b$ are not on the same side of $L$ then $a$ and $c$ are not on the same side of $L$

as a variant of the above. More generally, we should read the rule "if $\varphi_{1}, \varphi_{2}, \ldots, \varphi_{n}$ then $\psi$ " as the disjunction

either not $\varphi_{1}$, or not $\varphi_{2}$, or $\ldots$, or not $\varphi_{n}$, or $\psi$

and infer any disjunct once we know that the others are false. This is exactly the notion of direct consequence that we adopt: we take the set of direct consequences of a set of diagrammatic assertions to be the set obtained by closing the set under the inferences just described.

Let us spell out the details more precisely. For simplicity, we initially restrict our attention to propositional logic. A clause is simply a finite set of propositional literals; think of each clause as representing the associated disjunction. Let $S$ be a set of propositional clauses and let $\Gamma$ be a set of propositional literals. Take negation as an operation mapping literals to literals, that is, identify $\neg \neg p$ with $p$. We define the set of direct consequences of $\Gamma$ under $S$ to be the smallest set $\Gamma^{\prime}$ of literals that includes $\Gamma$ and is closed under the following rule: if $\left\{\varphi_{1}, \ldots, \varphi_{n}\right\}$ is a clause in $S$ and $\neg \varphi_{1}, \ldots, \neg \varphi_{n-1}$ are all in $\Gamma^{\prime}$, then $\varphi_{n}$ is in $\Gamma^{\prime}$. In other words, $\Gamma^{\prime}$ is obtained by starting with the literals in $\Gamma$ and applying the rule above to add literals, one at a time, until no more literals can be added. We adopt the understanding, however, that if $\Gamma^{\prime}$ contains an atomic formula and its negation, then it contains every literal; in other words, everything is a consequence of a contradiction.

We now provide an alternative characterization of the set $\Gamma^{\prime}$. Consider a sequent calculus formulation of intuitionistic logic [7, 59, with sequents of the form $\Pi \Rightarrow \varphi$, intended to denote that the set of hypotheses in $\Pi$ entails $\varphi$. Take the "contrapositive variants" of any clause $\left\{\varphi_{1}, \ldots, \varphi_{n}\right\}$ to be the sequents of the form $\left\{\neg \varphi_{1}, \ldots, \neg \varphi_{n-1}\right\} \Rightarrow \varphi_{n}$, again with the understanding that if $A$ is atomic then $\neg \neg A$ is replaced by $A$.

Proposition 3.1. Let $S$ be a set of clauses, and let $\Gamma, \theta$ be a set of propositional literals. The following are equivalent:

1. $\theta$ is a direct consequence of $\Gamma$ under $S$.

2. There is an intuitionistic proof of the sequent $\Rightarrow \theta$ from initial sequents that are either contrapositive variants of the clauses in $S$ or of the form $\Rightarrow \psi$, where $\psi$ is a formula in $\Gamma$. 
Proof. The implication from 1 to 2 is straightforward, since adding to $\Gamma^{\prime}$ the result of applying our rule of inference with one of the clauses in $S$ is equivalent to inferring the consequence of the implication given by a contrapositive variant of that clause. The fact that as soon as $\Gamma^{\prime}$ contains an atomic formula and its negation we take every literal to be a direct consequence follows from the fact that $\perp$, and hence every formula, is an intuitionistic consequence of an atomic formula and its negation.

Conversely, suppose there is an intuitionistic proof of $\Rightarrow \psi$ from the initial sequents described in 2 . Then by a version of cut-elimination theorem for the intuitionistic sequent calculus with axioms and additional rules (7, Theorem 2.4.5] or [59, Section 4.5.1]), there is a proof in which every cut formula is a literal. Since there are no other logical connectives in the initial sequents or conclusion, the only other rules used are the rules for negation and the "ex falso" rule $\Pi, \perp \Rightarrow \eta$.

We can therefore obtain the desired conclusion by proving the following claim:

Suppose $d$ is a proof of a sequent $\left\{\theta_{1}, \ldots, \theta_{n}\right\} \Rightarrow \eta$ from the initial sequents described in 2 , using only the negation rules, ex falso, and the cut rule restricted to literals. Then for any $\Gamma^{\prime \prime} \supseteq \Gamma$,

1. if $\theta_{1}, \ldots, \theta_{n}$ are in $\Gamma^{\prime \prime}$, then $\eta$ is in the closure of $\Gamma^{\prime \prime}$ under $S$; and

2. if $\eta$ is $\perp$ and $\theta_{1}, \ldots, \theta_{n-1}$ are in $\Gamma^{\prime \prime}$, then $\neg \theta_{n}$ is in the closure of $\Gamma^{\prime \prime}$ under $S$.

This can be proved by a straightforward induction on $d$. Suppose the the last inference of $d$ is the cut rule,

$$
\frac{\theta_{1}, \ldots, \theta_{n} \Rightarrow \alpha \quad \theta_{1}, \ldots, \theta_{n}, \alpha \Rightarrow \eta}{\theta_{1}, \ldots, \theta_{n} \Rightarrow \eta}
$$

If $\eta$ is not $\perp$, applying the inductive hypothesis to the left subproof yields that for any $\Gamma^{\prime \prime} \supseteq \Gamma$, if $\theta_{1}, \ldots, \theta_{n}$ are in $\Gamma^{\prime \prime}$, then $\alpha$ is in the closure of $\Gamma^{\prime \prime}$ under $S$. Applying the inductive hypothesis to the right subproof and $\Gamma^{\prime \prime}, \alpha$ yields that $\eta$ is in the closure of $\Gamma^{\prime \prime}, \alpha$ under $S$, and hence in the closure of $\Gamma^{\prime \prime}$ under $S$, as required. The case where $\eta$ is $\perp$ is similar.

Handling the other rules is straightforward. For example, if the last inference of $d$ is a left negation introduction, it is of the following form:

$$
\frac{\theta_{1}, \ldots, \theta_{n-1} \Rightarrow \alpha}{\theta_{1}, \ldots, \theta_{n-1}, \neg \alpha \Rightarrow \eta}
$$

In that case, the desired conclusions are obtained by applying the inductive hypothesis to the immediate subproof. 
In the statement of the last proposition, instead of taking all contrapositive variants of the clauses in $S$, one can equivalently take any one contrapositive variant of each clause in $S$, if we also add the following rule of double-negation elimination for atomic formulas:

$$
\frac{\Pi, \neg A \Rightarrow \perp}{\Pi \Rightarrow A}
$$

This has the net effect of making $\neg \neg A$ equivalent to $A$. But it is important to recognize that this is not the same as adding the law of the excluded middle, $A \vee$ $\neg A$, for atomic formulas. Indeed, this is exactly what is missing from the notion of a direct consequence. For example, suppose $S$ has rules "if $A$ and $B$ then $C$ " and "if $A$ and not $B$ then $C$." Then $C$ is certainly a classical propositional consequence of $\{A\}$ under these rules, since $C$ follows from both $B$ and from $\neg B$. But it is not a direct consequence. This distinction is what makes the notion of a direct consequence well-suited to modeling the diagrammatic inferences in the Elements. Euclid does explicitly introduce case splits when they are needed, and so any inference that requires considering different diagrammatic configurations, in an essential way, should not count as "reading off from the diagram." These case splits make all the difference: the next two propositions show that, in the propositional setting, they mark the difference between the complexity classes $\mathrm{P}$ and NP.

Proposition 3.2. Let $\Gamma$ be a set of literals and let $S$ be a set of clauses. The question "is $\theta$ a direct consequence of $\Gamma$ under $S$ ?" can be decided in time polynomial in the size of $\Gamma$ and $S$.

Proof. If the encoding of $\Gamma$ and $S$ have length $n$, they contain at most $n$ propositional variables. Starting with the literals in $\Gamma$, iteratively apply the closure rule using clauses in $S$, until $\theta$ is added, or the set becomes inconsistent, or no further rules can be applied. Each step of the iteration amounts to scanning through the clauses in $S$ and matching against literals already in $\Gamma^{\prime}$ to see whether a new literal can be added, and can be carried out in time polynomial in $n$. At each step, at least one literal is added the set $\Gamma^{\prime}$ of consequences, so the process terminates in at most $n+1$ steps.

Proposition 3.3. Suppose one augments intuitionistic logic with the following rule:

$$
\frac{\Pi, A \Rightarrow \eta \quad \Pi, \neg A \Rightarrow \eta}{\Pi \Rightarrow \eta}
$$

where $A$ is an atomic formula and $\Pi, \eta$ is a set of literals. Then a sequent $\Rightarrow \theta$ is provable from the initial sequents described in Proposition 3.1 if and only if $\theta$ is a classical consequence of $\Gamma$ together with the clauses in $S$. Hence, in the presence of such case splits, the problem of determining whether a literal is a consequence of $S$ is NP complete. 
Proof. Since the rule for case splits is classically valid, it is clear that if $\Rightarrow \theta$ is provable from the initial sequents 3.1, it is a classical consequence of $\Gamma$ together with the clauses in $S$.

Conversely, given $\Rightarrow \theta$, we can work backwards and apply case splits until at each node we have a sequent $\Pi \Rightarrow \theta$ such that for every propositional variable $p$ occurring in $\Gamma$ and $S$, either $p$ or $\neg p$ is in $\Pi$. If each such sequent is classically inconsistent with $\Gamma$ and the clauses in $S$, we obtain a proof of $\Rightarrow \theta$. Otherwise, at least one such $\Pi$ describes a truth assignment which is consistent with $\Gamma$ and $S$ but makes $\theta$ false, showing that $\theta$ is not a classical consequence of $\Gamma$ together with the clauses in $S$.

To prove the final claim in the lemma, let $S$ be any set of propositional clauses, and let $p$ be a new propositional variable. Then $S$ is satisfiable if and only if $p$ is not a classical consequence of $S$. The claim follows from the fact that the satisfiability of a set of propositional clauses is NP complete.

We now turn to the first-order setting. Suppose $S$ is a set of clauses, where now a clause is a finite set of first-order literals. Interpret these as universal axioms; that is, a clause $\left\{\varphi_{1}, \ldots, \varphi_{n}\right\}$ represents the universal closure of the associated disjunction. If $\Gamma$ is a set of literals, define the set $\Gamma^{\prime}$ of direct consequences of $\Gamma$ under $S$ as before, but now using arbitrary substitution instances of the clauses in $S$.

Focusing on $E$ in particular, we take the direct consequences of a set of diagrammatic assertions, $\Gamma$, to be the set of direct consequences of $\Gamma$ under the set of rules given in Section 3.4. Note that the language of $E$ has no function symbols. Since there are a fixed number of relation symbols, given $n$ variables ranging over points, lines, and circles, one can bound the number of literals involving these variables with a polynomial in $n$. The preceding propositions then show that our notion of direct consequence has the following desirable properties.

Theorem 3.4. Every direct consequence of a set of diagrammatic assertions is a first-order consequence of these assertions and the diagrammatic axioms.

Theorem 3.5. Any literal that is a classical consequence of a set of diagrammatic assertions and diagrammatic axioms can proved from those diagrammatic assertions in $E$.

Theorem 3.6. Let $\Gamma$ be a set of diagrammatic assertions involving at most $n$ points, lines, and circles. Whether or not a particular literal is a direct diagrammatic consequence of $\Gamma$ can be determined in time polynomial in $n$.

Note that "polynomial-time computable" need not mean feasible in practice. Since "between" is a ternary relation, with ten points, for example, we have to keep track of a thousand potential betweenness assertions. On the other hand, experiments described in Section 6 suggest that even the full set of quantifierfree consequences can be feasibly obtained for reasonable diagrams, so that our system should be practically implementable as well. 
We should also provide an account of what it means to be a direct metric consequence. It would be perhaps most faithful to Euclid to add a finite list of variants extending the list of axioms given in Section 3.5, allowing one to add equal segments to a segment in either order, and so on. But recognizing $\overline{a b}$ and $\overline{b a}$ as the same quantity, or $\overline{a b}+\overline{c d}$ and $\overline{c d}+\overline{a b}$ as the same quantity, should not need explicit justification; in general, a prover should be allowed to identify terms up to associativity, commutativity, and symmetric transformations without further comment. There are very simple computational devices that make this easy to implement in practice [15, and it is the kind of thing we (like Euclid) take for granted, and so we take these to be built into $E$.

In fact, we would not be doing too much damage to Euclid if we allowed any metric consequence of previous metric facts to be inferred in one step. This, too, has an easy computational implementation. As noted above, the theory is just the universal fragment of the theory of linearly ordered groups. Decision procedures for this theory have been studied extensively, and at the level of complexity one finds in Euclid's proofs, even the naive "Fourier-Motzkin" algorithm performs quite well in practice. (See [5] for an overview of such methods.)

Finally, to handle the transfer axioms, we allow the prover to assert, in one step, the conclusion of any single rule where the hypotheses are all direct diagrammatic or metric consequences of the available data, i.e. the diagrammatic and metric assertions in $\Gamma, \Delta$. Note that almost all these axioms can be described by clauses where exactly one of the literals is a metric assertion. (The exception is the third diagram-angle transfer axiom, which characterizes the notion of a "right angle" by stating an equivalence between two metric assertions in the context of some diagrammatic information. But this could be replaced by the Euclidean theorem that if a line is cut by a transversal, the adjacent angles add up to two right angles.) Sometimes Euclid takes certain metric information to be so clear from the diagram that he uses it without asserting it explicitly; these include, for example, our diagram-angle axiom 4, which asserts that different descriptions of the same angle have the same magnitude. In cases like that, one could modify our definition of "metric consequence" so that consequences of the diagram like these are added to the "store" of available metric hypotheses automatically.

This concludes our presentation of $E$. The fact that there is room to tinker with our notion of "direct consequence" by expanding or contracting the allowable inferences should help clarify the nature of our project. In order to show, in Section 5, that $E$ is sound and complete with respect to the relevant "ruler and compass" semantics, our one-step inferences have to be sound, and the full proof system has to be complete. This gives us a lot of latitude in defining the "one-step" inferences. The fact that soundness and completeness do so little to constrain our choice shows that we are trying to capture something more finegrained than the entailment relation for Euclidean geometry. Rather, we are trying to understand Euclidean proof, which requires an understanding of the sorts of inferences that are taken to be basic in the Elements. So, where Euclid draws an immediate conclusion from the data available in a proof, it should be 
possible to carry out that inference in one step, or at most a few steps, in our formal system. On the other hand, in cases where Euclid invokes a chain of steps to reach a conclusion, our system should not sanction that inference as "direct." The extent to which our system meets these constraints is the subject of the next section.

Ziegler 66] has shown that the notion of validity for ruler-and-compass semantics is undecidable. (His proof shows that the set of $\forall \exists \forall$ consequences of any finitely axiomatized fragment of the theory of real closed fields is undecidable. It is, however, still an open question whether the set of $\forall \exists$ consequences, which correspond to the geometric assertions that can be expressed in $E$, is decidable.) It is therefore interesting to note that, in principle, one can expand our notion of "direct consequence" dramatically and maintain decidability:

Theorem 3.7. The question as to whether a given literal is a first-order consequence of a finite set of literals and the set of all our diagrammatic, metric, and transfer axioms is decidable.

Proof. The problem is equivalent to determining whether a finite set $\Gamma$ of literals is consistent with the diagrammatic, metric, and transfer axioms. Write $\Gamma=$ $\Pi \cup \Theta$ where $\Pi$ consists of the diagrammatic literals and $\Theta$ consists of the metric literals. By splitting on cases, we can assume without loss of generality that for every diagrammatic atomic formula $\varphi$ involving the variables occurring in $\Gamma$, either $\varphi$ or $\neg \varphi$ is in $\Pi$. There are, moreover, only finitely many substitution instances of the axioms in question with the variables occurring in $\Gamma$. Modulo $\Pi$, all these axioms are equivalent to quantifier-free formulas over the metric sorts. We can then use a decision procedure for linear arithmetic to decide whether the resulting set of formulas, together with $\Theta$, is satisfiable.

This means that if decidability, soundness, and completeness for ruler-andcompass semantics were the only constraints, we could take proofs in $E$ to be nothing more than a sequence of construction steps, followed by "Q.E.D." (or "Q.E.F."). Due to the case splits, however, this naive algorithm runs in exponential time, and will be infeasible in practice.

\section{Comparison with the Elements}

In this section, we argue that $E$ provides an adequate modeling of the proofs in Books I-IV of the Elements, according to the criteria presented in Section 2 .

In Section 4.1 we focus on the language of the Elements, and in Section 4.2 we present some examples to illustrate how Euclid's proofs are represented in $E$. In Section 4.3. we explore some of the ways in which proofs in $E$ differ from Euclid's, and in Section 4.4 we compare our axiomatic basis to his. Finally, Section 4.5 provides a few more examples of proofs, some of a technical nature, that will be needed in our completeness proof in Section 5 . 


\subsection{Language}

We begin with a discussion of the language of the Elements. Since we have chosen a fairly minimal language for $E$, we need to fix some conventions for interpreting the less regimented and more expansive language in Euclid. For example, in the Elements, Euclid takes lines to be line segments, although postulate 2 ("to produce a finite straight line continuously in a straight line") allows any segment to be extended indefinitely. Distinguishing between finite segments and their extensions to lines makes it clear that at any given point in a proof, the diagrammatic information is limited to a bounded portion of the plane. But, otherwise, little is lost by taking entire lines to be basic objects of the formal system. So where Euclid writes, for example, "let $a$ and $b$ be points, and extend segment $a b$ to $c$," we would write "let $a$ and $b$ be distinct points, let $L$ be the line through $a$ and $b$, and let $c$ be a point on $L$ extending the segment from $a$ to $b$." Insofar as there is a fairly straightforward translation between Euclid's terminology and ours, we take such differences to be relatively minor.

Our basic diagrammatic terms include words like "on," "between," "inside," and "same side." It is worth noting that such words rarely occur explicitly in the Elements. Diagrammatic assertions are sometimes implicitly present in the result of a construction; in the example of the last paragraph, we use " $b$ is between $a$ and $c$ " to represent one of the outcomes of the diagrammatic construction. Euclid also sometimes uses the physical diagram to convey a diagrammatic assertion. For example, in the first proof in Section 2.1, the diagram shows that point $d$ is on $a b$. Diagrammatic information is also implicit in some of Euclid's more complicated locutions; for example, we need to analyze the Euclidean assertion " $a b c$ is a triangle" in terms of our more basic primitives. But, overall, it is remarkable how little diagrammatic information needs to be asserted in the text. One striking exception occurs in conveying the diagrammatic notion of being parallel (which we model with the diagrammatic predicate "does not intersect"): there is no way to represent the nonintersection of two lines in a diagram, and so Euclid uses the term "parallel" explicitly in Propositions 27-47 of Book I to make the assertion.

Modeling Euclid's limited use of explicit diagrammatic assertions has been a central goal in the design of $E$. Although one is allowed to enter diagrammatic assertions like " $a$ is between $b$ and $c$ " and " $a$ and $b$ are on the same side of $L$ " in proofs in $E$, the point is that often one does not need to. For example, if the fact that $b$ is between $a$ and $c$ is a direct consequence of diagrammatic assertions in the hypotheses of the theorem and previous construction steps, then, using a transfer axiom, one can simply assert that $\overline{a b}+\overline{b c}=\overline{a c}$, without further justification. Thus our choice of diagrammatic primitives was designed, primarily, to function internally, and keep track of the information that is required to license construction steps and explicit metric inferences.

(We remind you that, in contrast to Tarski's and Hilbert's axiomatizations of geometry, we use between $(a, b, c)$ to denote that $b$ is strictly between $a$ and $c$. This choice makes our translation, in Section 5 , to a formal system based on Tarski's axioms slightly more complicated. On the other hand, it does seem to 
correspond more closely to Euclidean practice; see the discussion in Section 2.5. Interestingly, as noted in Section 6 below, it also seems to provide better performance in implementations.)

Having discussed our choice of diagrammatic primitives, we comment briefly on our modeling of metric assertions. In the Heath translation of Euclid, one finds phrases like "the base $a b$ is equal to the base de," "angle $a b c$ is greater than angle def," and "angles $a b c, c b d$ are equal to two right angles." We model these in our formal system with the metric assertions $\overline{a b}=\overline{d e}, \angle a b c>\angle d e f$, and $\angle a b c+\angle c b d=$ right-angle + right-angle. In reasoning about such quantities, Euclid uses basic properties of an ordered group. For example, in the middle of the text of Proposition I.13, we find:

... since the angle $d b a$ is equal to the two angles $d b e, e b a$, let the angle $a b c$ be added to each; therefore the angles $d b a, a b c$ are equal to the three angles $d b e, e b a, a b c$. But the angles $c b e, e b d$ were proved equal to the same three angles; and things which are equal to the same thing are equal to one another; therefore the angles cbe, ebd are also equal to the angles $d b a, a b c$. [16, p. 275]

In our system, this sequence of assertions would be represented as follows:

$$
\begin{aligned}
& \angle d b a=\angle d b e+\angle e b a \\
& \angle d b a+\angle a b c=\angle d b e+\angle e b a+\angle a b c \\
& \angle c b e+\angle e b d=\angle d b e+\angle e b a+\angle a b c \\
& \angle c b e+\angle e b d=\angle d b a+\angle a b c
\end{aligned}
$$

In the example, the first assertion is a metric consequence of diagrammatic information, namely that the point $e$ is in the interior of the angle $d b a$. The third assertion is echoed from earlier in the proof, and the other two are obtained using axioms of equality. Even though Euclid does not use a symbol for addition or the word "sum," it is clear from the text that his usage of magnitudes "taken together" is modeled well by the modern notions.

Other locutions found in Euclid can be modeled as "definitional extensions" of $E$. For example, consider the phrase "let $a b c$ be a triangle." Assuming we take this to mean a nondegenerate triangle, we parse this as saying that $a, b$, and $c$ are points, and there are lines $L, M$, and $N$, such that $a$ and $b$ are on $L$ but $c$ is not, $b$ and $c$ are on $M$ but $a$ is not, and $c$ and $a$ are on $N$ but $b$ is not. Furthermore, the Euclidean phrase "let $a b$ be produced to $d$ " involves picking a point $d$ on $L$ extending the segment from $a$ to $b$, and so on. Adequate modeling of Euclidean talk of triangles thus involves introducing mild forms of "syntactic sugar" to $E$.

When it comes to areas, we have only introduced a primitive for the area of a triangle. Books I to IV also deal with areas of parallelograms (including squares and rectangles) and, in the proof of Proposition I.35, a trapezoid. One could introduce a new primitive to denote the area of a convex quadrilateral (convexity can be defined in the language of $E$ ), with appropriate axioms. Alternatively, one can define the area of a convex quadrilateral abcd to be the sum of the 
areas of triangle $a b c$ and $a c d$, and then introduce the requisite properties as "derived rules." Extending $E$ to handle the area of arbitrary convex polygons (that is, convex polygons with an arbitrary number of sides) would require a more dramatic extension, but this notion never arises in the Elements.

One can prove in $E$ that one can pick an arbitrary point in a triangle, say, or in a rectangle, but these facts require proof, even though they are diagrammatically obvious. To our knowledge, however, Euclid never does this. To model subsequent developments in geometry, one would probably need to extend $E$ with a uniform treatment of convex figures.

There are a number of concepts found in later books of the Elements that we have not incorporated into $E$. For example, Book V introduces the notion of multiples and ratios; propositions in Book VI refer to arbitrary polygons; and Book VII, which introduces elementary number theory, refers to arbitrary (finite) collections of numbers. It would be interesting to extend $E$ to model the Euclidean treatment of such concepts as well.

In our formulation of $E$, one is allowed to carry out arguments by case splits on an atomic formula. Case splits in Euclid can be slightly more expressive; for example, knowing that angles $a b c$ and $a b d$ do not coincide, Euclid may consider the two cases $a b c<a b d$ and $a b c>a b d$. We would model this by first splitting on the assertion $\angle a b c<\angle a b d$; then in the case $\angle a b c \nless \angle a b d$, we would employ a second case split on the predicate $\angle a b c=\angle a b d$, the positive instance which has already been ruled out. We maintain that all case arguments occurring in the first four books of the Elements can be obtained in this way, using a sequence of atomic splits to obtain an exhaustive list of possibilities (e.g. if $a$ is a point not on a line $L$, then another point $b$ is either on the same side of $L$ as $a$, on $L$, or on the opposite side of $L$ ), some of which are ruled out immediately (implying $\perp$, and hence the desired conclusion right away). Once again, mild forms of "syntactic sugar" would allow one to express these case splits more compactly, resulting in proofs in $E$ that more closely model the ones in Euclid.

When different diagrammatic configurations are possible, Euclid will sometimes prove only one case. Often this case is truly "without loss of generality," which is to say, the other case (or cases) are entirely symmetric. In $E$, strictly speaking, we would have to repeat the proof; but one could introduce a syntactic term, "similarly," to denote such a repetition. However, as Heath points out repeatedly, Euclid often proves only the most difficult case of a proposition and omits the others, even though they may require a different argument; indeed,

much of Proclus' commentary is devoted to supplying proofs of the additional cases (see, for example, the notes to Propositions 2, 7, 25, and 35 in 16, Book I]). Of course, in cases like this $E$ requires the full argument. There is no reasonable syntactic account of the phrase "left to reader," and we do not purport to provide one.

\subsection{Examples of proofs in $E$}

In this section, we provide some examples of proofs in our formal system $E$, assuming the kinds of "syntactic sugar" described in the last section. We include 
diagrams to render the proofs intelligible, but we emphasize that they play no role in the formal system. To improve readability, we use both the words "Have" and "Hence" to introduce assertions, generally using "Have" to introduce new metric assertions that are inferred from the diagram, and "Hence" to introduce assertions that follow from previous metric assertions. But these words play no role in the logical system; all that matters are the actual assertions that follow. For the sake of intelligibility, we also sometimes add comments, in brackets. Once again, these play no role in the formal proof. Since the point of this exercise is to demonstrate that proofs in $E$ are faithful to the text of the Elements, we recommend comparing our versions with Euclid's.

Proposition 1 of Book I requires one, "on a given straight line, to construct an equilateral triangle."

\section{Proposition I.1.}

Assume $a$ and $b$ are distinct points. Construct point $c$ such that $\overline{a b}=\overline{b c}$ and $\overline{b c}=\overline{c a}$.

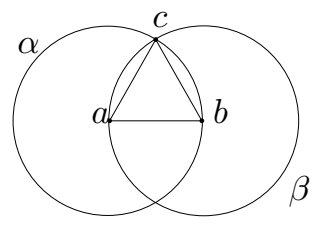

Proof. Let $\alpha$ be the circle with center $a$ passing through $b$.

Let $\beta$ be the circle with center $b$ passing through $a$.

Let $c$ be a point on the intersection of $\alpha$ and $\beta$.

Have $\overline{a b}=\overline{a c}$ [since they are radii of $\alpha$ ].

Have $\overline{b a}=\overline{b c}$ [since they are radii of $\beta$ ].

Hence $\overline{a b}=\overline{b c}$ and $\overline{b c}=\overline{c a}$.

Q.E.F.

The hypotheses tell us only that $a$ and $b$ are distinct points, but this is enough to license the construction of $\alpha$ and $\beta$, by rule 2 of the construction rules for lines and circles. Rule 5 of diagram rules for intersections gives us the diagrammatic fact that $\alpha$ and $\beta$ intersect. Rule 6 of the construction rules for intersection then allows us to pick a point of intersection. Rule 3 of the diagram-segment transfer axioms then allows us to conclude that the given segments are equal, since they are radii of the two circles. Using metric inferences (the symmetry of line segments and transitivity of equality) gives us that $a b=b c=c a$.

Our proof does not establish, per se, that $c$ is distinct from $a$ and $b$, and this $i s$ an assumption that Euclid uses freely when applying the theorem. Fortunately, this is an easy metric consequence.

Auxiliary to Proposition I.1.

Assume $a$ and $b$ are distinct points, $\overline{a b}=\overline{b c}$, and $\overline{b c}=\overline{c a}$.

Then $c \neq a$ and $c \neq b$. 
Proof. Suppose $c=a$.

Hence $a=b$.

Contradiction.

Hence $c \neq a$.

Suppose $c=b$.

Hence $a=b$.

Contradiction.

Hence $c \neq b$.

Q.E.D.

To show that $c$ is distinct from $a$, we suppose, to the contrary, that $c=a$. Then direct metric inferences give us $\overline{a c}=0, \overline{a b}=0$, and $a=b$, which is a contradiction. (We use the word "Contradiction" for "Hence False.") The fact that $c$ and $b$ are distinct is proved in the same way.

A more faithful rendering of the proposition might assume "Let $a$ and $b$ be distinct points on a line, $L$," and then also construct the remaining lines $M$ and $N$ of the triangle. If one uses Proposition I.1 as we initially stated it, one can simply construct $M$ and $N$ afterwards. Euclid also, however, sometimes needs the fact that $c$ is not on the line determined by $a$ and $b$. Once again, by $E$ 's lights, this requires a short argument.

\section{Auxiliary to Proposition I.1.}

Assume $a$ and $b$ are distinct points, $a$ is on $L, b$ is on $L$, and $\overline{a b}=\overline{b c}$ and $\overline{b c}=\overline{c a}$. Then $c$ is not on $L$.

Proof.

Suppose $c$ is on $L$.

Suppose $a$ is between $c$ and $b$.

Hence $\overline{c a}<\overline{b c}$. Contradiction.

Suppose $c=a$.

Hence $a=b$. Contradiction.

Suppose $c$ is between $a$ and $b$.

Hence $\overline{c a}<\overline{a b}$. Contradiction.

Suppose $c=b$.

Hence $a=b$. Contradiction.

Suppose $b$ is between $a$ and $c$.

Hence $\overline{a b}<\overline{b c}$. Contradiction.

Contradiction.

Q.E.D.

If $a$ and $b$ are distinct points on a line, Euclid often splits implicitly or explicitly on cases depending on the position of a point $c$ relative to $a$ and $b$. Strictly speaking, the proof above could be expressed as a sequence of four nested case splits on atomic formulas. As noted in the previous section, we can take the proof above to rely on notational conventions, for readability.

When it is easy to rule out some cases, Euclid often does not say anything at all, where our rules may require a line or two. The fact that Euclid doesn't say 
anything to justify the nondegeneracy of the triangle constructed in Proposition I.1, where $E$ requires some (easy but) explicit metric considerations, is a more dramatic difference, and is discussed in Section 4.3. There, in fact, we note that in the proof of Proposition I.9, Euclid seems to need a slight strengthening of our Proposition I.1, which asserts that $c$ can be chosen on either side of the $L$ through $a$ and $b$. This is easily obtained using rule 8 instead of rule 6 of the construction rules for intersections; one only needs to take the trouble to make the stronger assertion.

Proposition 2 in Book I of the Elements is surprisingly complicated given that it occurs so early. It is a construction, requiring one "to place at a given point a straight line equal to a given straight line," that is, to copy a segment to a given point. This time, we leave it to you to check that the assertions are justified by our rules and our notion of direct inference, providing some hints in the bracketed comments. To simplify the exposition, we appeal to a version of Proposition I.1 with the additional distinctness claim.

\section{Proposition I.2.}

Assume $L$ is a line, $b$ and $c$ are distinct points on $L$, and $a$ is a point distinct from $b$ and $c$.

Construct point $f$ such that $\overline{a f}=\overline{b c}$.

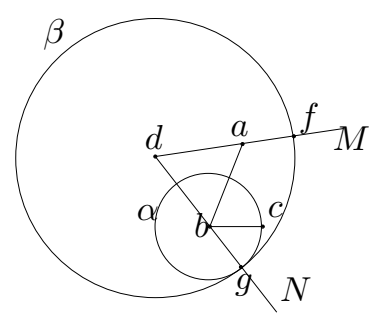

Proof. By Proposition I.1 applied to $a$ and $b$, let $d$ be a point such that $d$ is distinct from $a$ and $b$ and $\overline{a b}=\overline{b d}$ and $\overline{b d}=\overline{d a}$.

Let $M$ be the line through $d$ and $a$.

Let $N$ be the line through $d$ and $b$.

Let $\alpha$ be the circle with center $b$ passing through $c$.

Let $g$ be the point of intersection of $N$ and $\alpha$ extending the segment from $d$ to $b$.

Have $\overline{d g}=\overline{d b}+\overline{b g}$.

Hence $\overline{d g}=\overline{d a}+\overline{b g}[$ since $\overline{d a}=\overline{d b}]$.

Hence $\overline{d a}<\overline{d g}$.

Let $\beta$ be the circle with center $d$ passing through $g$.

Hence $a$ is inside $\beta$ [since $d$ is the center and $\overline{d a}<\overline{d g}$ ].

Let $f$ be the intersection of $\beta$ and $M$ extending the segment from $d$ to $a$.

Have $\overline{d f}=\overline{d a}+\overline{a f}$.

Have $\overline{d f}=\overline{d g}$ [since they are both radii of $\beta]$.

Hence $\overline{d a}+\overline{a f}=\overline{d a}+\overline{b g}$.

Hence $\overline{a f}=\overline{b g}$. 
Have $\overline{b g}=\overline{b c}[$ since they are both radii of $\alpha$ ].

Hence $\overline{a f}=\overline{b c}$.

Q.E.F.

Notice that the last construction step requires knowing that $a$ is inside $\beta$. We obtain this, in our proof, using simple metric considerations. We discuss this fact in the next section.

Let us consider one more example. You may wish to compare the following rendering of Proposition I.10 to the one given in Section 2.1. Once again, to simplify the exposition, we appeal to a version of Proposition I.1 with the additional noncollinearity claim. The proof also appeals to Proposition I.9, which asserts that an angle $a c b$ can be bisected. We take this to be the assertion that there is a point $e$ such that $\angle a c e=\angle b c e$; with the further property that if $M$ is the line through $c$ and $a$, and $N$ is the line through $c$ and $b$, then $e$ and $b$ are on the same side of $M$, and $e$ and $a$ are on the same side of $N$. The last requirement could be expressed more naturally with the words " $e$ is inside the angle $a c b$," though that locution does not make $M$ and $N$ explicit. This requirement rules out choices of $e$ on the other side of $c$ which satisfy the same metric conditions.

\section{Proposition I.10.}

Assume $a$ and $b$ are distinct points on a line $L$.

Construct a point $d$ such that $d$ is between $a$ and $b$ and $\overline{a d}=\overline{d b}$.

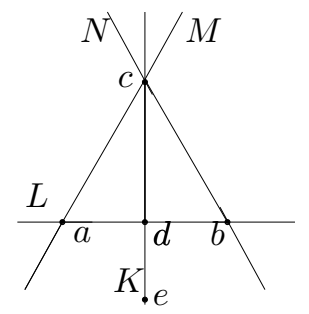

Proof. By Proposition I.1 applied to $a$ and $b$, let $c$ be a point such that $\overline{a b}=\overline{b c}$ and $\overline{b c}=\overline{c a}$ and $c$ is not on $L$.

Let $M$ be the line through $c$ and $a$.

Let $N$ be the line through $c$ and $b$.

By Proposition I.9 applied to $a, c, b, M$, and $N$, let $e$ be a point such that $\angle a c e=\angle b c e, b$ and $e$ are on the same side of $M$, and $a$ and $e$ are on the same side of $N$.

Let $K$ be the line through $c$ and $e$.

Let $d$ be the intersection of $K$ and $L$.

Have $\angle a c e=\angle a c d$.

Have $\angle b c e=\angle b c d$.

By Proposition I.4 applied to $a, c, d, b, c$, and $d$ have $\overline{a d}=\overline{b d}$.

Q.E.F. 

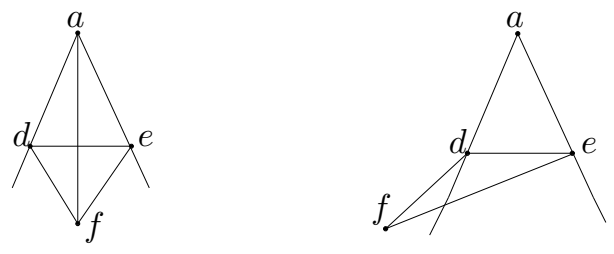

Figure 8: Two cases for Proposition I.9 considered in $E$.

As noted in Section 2.1, when applying Proposition I.9, Euclid immediately takes $d$ to be the point of intersection; we need to bisect the angle and then choose the intersection explicitly. A direct diagrammatic inference yields the fact that the two lines intersect: the triple incidence axioms imply that points $a$ and $b$ are on opposite sides of $K$, which serves as the hypothesis to intersection rule 1 . We also need to note that the angles acd and $b c d$ are then the same as angles ace and bce, which is justified by metric rule 6 . The fact that $d$ is between $a$ and $b$ is again the result of a direct diagrammatic inference, using Pasch inference 4 .

There are some cases where the extent to which formal proofs in $E$ match Euclid's is particularly impressive. For example, Proposition 1 of Book III is "to find the center of a given circle." This may seem strange, since Euclid's definitions seem to suggest that every circle comes "equipped" with its center 7 but the proposition makes it clear that we can be "given" a circle on its own. The fact that we use a relation symbol rather than a function symbol to pick out the center of a circle makes our formalization of Proposition III.1 as $\exists a$.center $(a, \gamma)$ perfectly natural, and the proof is essentially Euclid's.

For another example, Proposition 2 of Book III shows that circles are convex - more precisely, that the chord of a circle lies inside the circle. This, too, is somewhat surprising, since that fact seems to be as obvious as anything one is allowed to "read off" from a diagram. But in $E$, one needs a proof using metric considerations, as in Euclid. Thus $E$ can help "explain" some puzzling features of the Elements.

\subsection{Departures from the Elements}

In this section, we discuss some instances where proofs in the Elements do not accord as well with the rules of $E$. Perhaps unsurprisingly, the most common type of departure involves cases where Euclid's arguments are not detailed enough, by the standards of $E$. Among these cases, two situations are typical: first, Euclid is sometimes content to consider only one case when $E$ demands a case analysis, and, second, Euclid sometimes reads directly from the diagram a geometric relation which in $E$ must be licensed by a transfer rule. We will consider examples of each, in turn.

\footnotetext{
${ }^{7}$ We are grateful to Henry Mendell for pointing this out.
} 

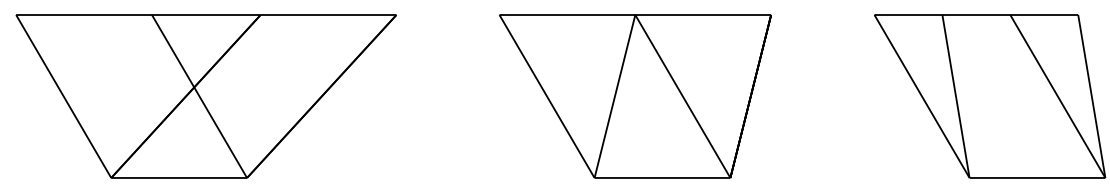

Figure 9: The three cases for Proposition I.35.

As pointed out in Section 3.2. Euclid occasionally reasons by cases to establish a proposition. When Euclid carries out such a case analysis, $E$ typically provides a natural account of the proof. But when $E$ demands a case analysis, Euclid does not always provide one. For an example, consider Euclid's proof of Proposition 9 in book I. The proposition is a problem which demands the construction of an angle bisector (see Figure 8). After constructing equal segments $a d$ and $a e$ on the two sides of the given angle (with vertex $a$ ), Euclid joins $d$ and $e$ and constructs on the segment the equilateral triangle $d f e$. The vertex $f$ of the triangle is then joined with the vertex $a$ of the angle, and it is then argued that this segment bisects the angle. Euclid takes it as given that the point $f$ falls within the angle. In $E$, however, one cannot. Though one may stipulate that $f$ falls on the side of the segment de opposite the point $a$, one cannot assume anything about $a$ 's position with respect to the sides of the angle. One must consider the cases where $f$ falls on or outside the angle, and show that they are impossible 8

Another place where Euclid falls short of meeting E's standards for case analysis is Proposition I.35. Whereas with Proposition I.9 the need for a case analysis arises within the construction, with Proposition I.35 one must start the proof with a case analysis (see Figure 9). Euclid's statement of the proposition is too general for the proof which follows. The proposition underlies the familiar formula that the area of a parallelogram is the product of its base and height. It asserts, specifically, that two parallelograms which have the same base and are bounded by the same parallel lines have the same area. The proof in the Elements, however, establishes a weaker result, in which the parallelograms satisfy another condition: the nonintersection of the sides opposite the common base of the parallelograms. Euclid groups together into one case the different ways the sides opposite the base can relate to one another positionally. But the containment relations which license Euclid's steps in his proof do not generalize to the other cases, which really require separate proofs.

Proclus, in fact, commented on Euclid's cavalier attitude toward cases in

\footnotetext{
${ }^{8}$ Vaughan Pratt has pointed out to us the contrapositive of Proposition 7 shows that if $a d$ is equal to $a e, d f$ is equal to $e f$, and $d$ and $e$ are distinct, then $d$ and $e$ cannot lie on the same side of af. This immediately rules out two of the cases. But Euclid typically carries out an explicit reductio when he needs the contrapositive form of a prior proposition. Thus, if that is the proof one has in mind, $E$ requires one to do the case split and apply Proposition 7 explicitly.
} 
Propositions I.9 and I.35, and furnished proofs for some of the cases Euclid neglected. Thus $E$ is better understood as a codification of the more critical attitude towards cases found in Proclus's commentary. It is an interesting question as to why Euclid is less rigorous in cases like these. One possible explanation is given by Heath's observation that Euclid only worries about the most difficult case. Another, which would apply to I.9 but not I.35, is that the norms governing the physical construction of diagrams automatically rules out certain possibilities for Euclid 9

As with $E$ 's rules for case analysis, its transfer rules can be understood as the articulation of standards observed intermittently in the Elements. In some constructions, the possibility of a certain step depends on metric facts assumed of the configuration. On such occasions, $E$ requires that a metric-to-diagram rule be invoked. Euclid sometimes recognizes the need for such justifications, and sometimes does not.

One place where he does not is in Proposition 2 of Book I. In terms of the $E$ proof given in Section 4.2, Euclid does not provide any argument that the point $a$ has to lie within the circle $\beta$. The diagrammatic information in the proof regarding $a$ with respect to $\beta$, however, does not alone imply it. The metric fact that $d a<d g$ must be added to the proof for the position of $a$ inside $\beta$ to be forced. The $E$ proof of Proposition 2 thus contains a few lines not present in Euclid's proof.

Euclid does explicitly state one metric-to-diagram rule: the famous parallel postulate. The postulate allows Euclid to speak of an intersection point between two lines - a diagrammatic piece of data - given metric data about a configuration in which the lines are embedded. Accordingly, in Propositions I.44 and II.10 Euclid invokes it to justify the introduction of certain intersection points. Strangely, however, a similar justification is needed for intersection points appearing in Euclid's proofs of Propositions I.42 and I.45, but Euclid does not provide it. He simply takes the intersection points to exist without mentioning the parallel postulate. The reasons for this inconsistency are not immediately apparent. The arguments which are lacking in I.42 and I.45 are more complicated than those included in I.44 and II.10. Perhaps Euclid did not want to complicate his exposition, or perhaps it was just an oversight. In any case, in $E$, one must invoke the parallel postulate in the proofs of all four propositions.

We close this section with a discussion of another interesting difference between $E$ and Euclid. This time, it is an instance where, by $E$ 's lights, Euclid does too much. At issue are the identity conditions of circles. Euclid's definition reads as follows:

A circle is a plane figure contained by one line such that all the

\footnotetext{
${ }^{9}$ Such norms would enforce what Manders terms diagram discipline. The idea is as follows. Though physical rulers and compasses cannot produce perfectly straight lines and circles, a geometer trained in diagram discipline can be trusted to produce approximately straight lines and circles in his diagrams. For $f$ to lie on or outside the angle dae in I.9, however, one or more of the circles used in the construction of $f$ would have to be dramatically non-circular. Euclid would thus be justified in disregarding the case as a possibility. See [32, section 3.1, p. 131], and also the discussion of case branching in 31 Section 1.4, p. 95].
} 
straight lines falling upon it from one point among those lying within the figure equal one another; and the point is called the center of the circle. [16, pp. 153-154]

In $E$ this definition translates into diagram-segment transfer Rules 2, 3, and 4 . The function of the Rule 2 is to fix the construction of a circle from a given length as unique. In fixing it as a rule in $E$, we take it to express Euclid's definition directly. Euclid, however, feels that it is at least conceivable that two distinct circles with equal radii be constructed from the same center, for in Proposition III.5 he proves that such a configuration is impossible. From this result Rule 2 then follows immediately.

Thus, with Proposition III.5 Euclid requires a proof for something which one can assume without proof in $E$. There is nothing, however, about the general structure of $E$ which forces this difference; we could have replaced our Rule 2 with a rule that licenses the key diagrammatic inference in Euclid's proof of III.5. Such a rule, however, would be complicated, and rather than assume it we have decided to treat circles as uniquely defined by a center and a length. Instead, our Rule 2 conforms better to the modern conception of a circle as the set of points which lie a fixed distance from a given center.

\subsection{Euclid's postulates and common notions}

Since the Elements is presented as an axiomatic development, it it is worth considering Euclid's postulates and common notions, to see how they line up with the fundamental rules of $E$. In the Heath translation [16, p. 154-155], the postulates are as follows:

1. To draw a straight line from any point to any point.

2. To produce a finite straight line continuously in a straight line.

3. To describe a circle with any centre and distance.

4. That all right angles are equal to one another.

5. [The parallel postulate; see Section 3.5]

Postulates 1 and 3 are the construction rules of $E$ for lines and circles. Postulate 2 does not have a direct translation in our system, given that we take all our lines to be "indefinitely extended"; but since Euclid will use this, say, to extend a segment $a b$ to a point $c$, it essentially corresponds to construction 4 for points. Our remaining construction rules let us choose "arbitrary points" or label points of intersection. Euclid doesn't say anything more about this; he just does it. As noted in Section 3.6. Euclid's Postulate 4 essentially corresponds to our diagram-angle transfer axiom 3. Similarly, Postulate 5 is our diagram-angle transfer axiom 5 .

Euclid's common notions are as follows [16, p. 155]:

1. Things which are equal to the same thing are also equal to one another. 
2. If equals be added to equals, the remainders are equal.

3. If equals be subtracted from equals, the remainders are equal.

4. Things which coincide with one another are equal to one another.

5. The whole is greater than the part.

These, for the most part, govern magnitudes; in our formulation, they are therefore subsumed by the laws that govern the metric sorts, together with the transfer axioms that relate the diagrammatic notions of "adding," "subtracting," and "being a part of" to the operations on magnitudes. For example, common notions 1 and 2 are equality rules, and common notion 3 is the cancellation axiom, modulo what it means to combine magnitudes in diagrammatic terms. Our first diagram-segment transfer axiom explains what it means to add adjacent segments; our second diagram-angle transfer axiom explains what it means to add adjacent angles; our second diagram-area transfer axiom explains what it means to combine the areas of adjacent triangles. In each case, one can take the diagrammatic configurations representing the component magnitudes to be the "parts" of the diagram configurations representing the sum. In that case, the last common notion, 5, corresponds to the fact that nontrivial segments, angles, and areas are positive, as given by the corresponding transfer axioms.

Thus, Euclid's postulates correspond to some of our construction rules and transfer inferences, and the common notions correspond to metric inferences and other transfer inferences. The remainder of our construction rules, and all our diagram inferences, are then subsumed under what Euclid takes to be implicit in the definitions and the meanings of the undefined terms. It is, perhaps, regrettable that there is not a cleaner mapping from our axioms to Euclid's. But, as the discussion above indicates, even a simple principle like "the whole is greater than the part" assumes an understanding of how wholes and parts can be recognized in a diagram, and it is this implicit understanding that we have tried to spell out with the rules of $E$.

\subsection{Additional proofs}

In this section, we provide three additional theorems of $E$, which are needed for the completeness proof in the next section. The first is Euclid's Proposition I.12. Here, the phrase " $M$ is perpendicular to $L$ " masks implicit references to points $p, d, a$ such that $p$ is on $M, d$ is on both $M$ and $L, a$ is on $L$, and angle $p d a$ is a right angle.

Proposition I.12.

Assume point $p$ is not on line $L$.

Construct a line $M$ through $p$ which is perpendicular to $L$. 


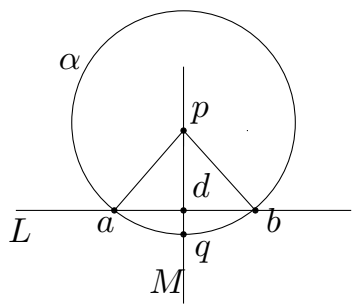

Proof. Let $q$ be a point on the opposite side of $L$ from $p$.

Let $\alpha$ be the circle through $q$ with center $p$.

Let $a$ and $b$ be the points of intersection of $L$ and $\alpha$.

By Proposition I.10, let $d$ bisect segment $a b$.

Let $M$ be the line through $p$ and $d$.

By Proposition I.8 applied to triangles $p d a$ and $p d b$, we have $\angle p d a=\angle p d b$.

Hence $\angle p d a$ is a right angle.

Q.E.F.

The proof is almost identical to Euclid's. Notice that it is the fourth diagram intersection rule that licenses the assertion that $L$ and $\alpha$ intersect.

The next two propositions are of a purely technical nature. The first shows how a construction in $E$ can depend on a case split (see footnote 4 ). Once again, we have taken some liberties with the wording. Reference to the "line through $p$ and $s$, for example, masks a reference to a variable for a line on which $p$ and $s$ both lie.

\section{Technical Proposition 1.}

Assume $p \neq q$ are on the same side of line $L$. Construct points $r, s, t$ such that

1. $s, t$ are on $L$,

2. $r$ is the intersection of the line through $p$ and $s$ and the line through $q$ and $t$.

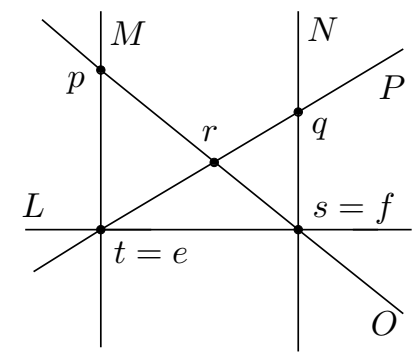

Proof. By Proposition I.12, let $M$ be a line through $p$ perpendicular to $L$, intersecting $L$ at $e$.

By Proposition I.12, let $N$ be a line through $q$ perpendicular to $L$, intersecting $L$ at $f$. 
Suppose $e \neq f$.

Hence $M$ and $N$ are parallel.

Let $s=f$.

Let $t=e$.

Let $O$ be the line through $p$ and $s$.

Let $P$ be the line through $q$ and $t$.

Let $r$ be the intersection of $O$ and $P$.

Then $r, s, t$ satisfy 1 and 2 .

Suppose $e=f$.

Let $s$ be a point on $L$ distinct from $e$.

Let $t$ be a point on $L$ extending the segment from $s$ to $e$.

Let $O$ be the line through $p$ and $s$.

Let $P$ be the line through $q$ and $t$.

Let $r$ be the intersection of $O$ and $P$.

Then $r, s, t$ satisfy 1 and 2 .

Q.E.F.

In the first case, a diagram inference tells us that $p$ and $t$ are on the same side of $M$ (since otherwise $N$ and $M$ would intersect). A triple-incidence rule, applied to $L, M$, and $N$ then tells us that $q$ and $t$ are on opposite sides of $O$, which licenses the fact that $O$ and $P$ intersect. The second case actually requires a case distinction on the position of $p$ and $q$ along the perpendicular, at which point, the Pasch rules provide enough information to license the fact that $O$ and $P$ intersect.

\section{Technical Proposition 2.}

Assume line $L$ and points $p, q, r, s, t$ satisfy the conclusions of the previous proposition.

Then $p$ and $q$ are on the same side of $L$.

In fact, this is a direct diagrammatic inference, using the Pasch rules.

\section{Completeness}

In this section, we sketch a proof that $E$ is complete for a modern semantics appropriate to the Elements. This semantics is presented in Section 5.1, and the completeness proof is presented in Sections 5.2 5.4 .

\subsection{The semantics of ruler-and-compass constructions}

Thanks to Descartes, Euclid's points, lines, and circles can be interpreted, in modern terms, as points, lines, and circles of the Euclidean plane, $\mathbb{R} \times \mathbb{R}$. It is straightforward to show that all the constructions and inference rules of $E$ are valid for this semantics. $E$ is not, however, complete for this semantics: all of Euclid's constructions, and hence all constructions of $E$, can be carried out with a ruler and compass, and Galois theory tells us that no ruler-andcompass construction can trisect a sixty degree angle [23, p. 240]. In particular, 
$E$ cannot prove that there exists an equilateral triangle and a trisection of one of its angles. The negation of this statement is a universal statement, and so can also be expressed in $E$. This shows that there is an existential statement that can neither be proved nor refuted in $E$, showing that $E$ is not syntactically complete, either.

Fortunately, there is a better semantics for the Elements. An ordered field is said to be Euclidean if every nonnegative element has a square root. Taking square roots essentially allows one to construct the intersection of a line and a circle, and conversely. Say that a sequent of $E$ is valid for ruler and compass constructions if its universal closure is true in every plane $F \times F$, where $F$ is a Euclidean field, under the usual cartesian interpretation of the primitives of $E$. Our goal in this section is to outline a proof of the following:

Theorem 5.1. A sequent $\Gamma \Rightarrow \exists \vec{x}$. $\Delta$ is valid for ruler-and-compass constructions if and only if it is provable in $E$.

Once again, the "if" direction, asserting that $E$ is sound for ruler-andcompass constructions, is straightforward. We will therefore focus on establishing completeness. A direct proof would involve assuming that a given sequent is not provable in $E$, and then constructing a Euclidean field in which that sequent is false. But given $E$ 's restricted logic, the details would be tricky, and our job will be much easier if we build on previous work. Tarski [57] gave a sound and complete axiomatization not only of the full Euclidean plane, but also of the fragment that is valid for ruler-and-compass constructions. It is therefore sufficient to show that $E$ is complete with respect to Tarski's axiomatization of the latter.

There are, however, obstacles to this approach. For one thing, Tarski's axiomatization of geometry uses only one sort, namely points, and two primitives, for betweenness and equidistance, as described below. So interpreting statements of $E$ in Tarski's system and vice-versa involves a change of language. A more serious obstacle is that Tarski uses full first-order logic, in contrast to the very meager fragment that is allowed in $E$. So knowing that a statement is provable in Tarski's system is not a priori helpful, since there will generally be no line-by-line interpretation of this proof in $E$.

Below, however, we will show that with a modicum of tinkering, Tarski's axioms can be expressed in a restricted form, namely, as a system of geometric rules. We will then invoke a cut elimination theorem, due to Sara Negri, that shows that if a sequent of suitably restricted complexity is provable in the system, there is a proof in which every intermediate sequent is also of restricted complexity. This will allow us to translate proofs in Tarski's system to proofs in $E$.

More precisely, we will craft a slight variant, $T$, of Tarski's system, which is sound and complete for ruler-and-compass constructions, and enjoys some nice proof-theoretic properties. We will define a translation $\pi$ from sequents of $E$ to sequents of $T$, and a re-translation $\rho$ in the other direction. Ultimately, we will show that the systems and translations involved have the following properties: 
1. If $\Gamma \Rightarrow \exists \vec{x}$. $\Delta$ is valid for ruler and compass constructions, then $T$ proves $\pi(\Gamma \Rightarrow \exists \vec{x} . \Delta)$.

2. If $T$ proves $\pi(\Gamma \Rightarrow \exists \vec{x} . \Delta)$, then $E$ proves $\rho(\pi(\Gamma \Rightarrow \exists \vec{x} . \Delta))$.

3. If $E$ proves $\rho(\pi(\Gamma \Rightarrow \exists \vec{x} . \Delta))$, then $E$ proves $\Gamma \Rightarrow \exists \vec{x} . \Delta$.

This yields the desired completeness result. Since many of the details are straightforward, we will be somewhat sketchy; additional information can be found in Dean's MS thesis [14].

In fact, we will not interpret the area (" $\triangle$ ") function of $E$ or the functions and relations on the area sort; so we only establish completeness for theorems that do not involve areas. Defining an adequate notion of area in Tarski's system requires a fair amount of work, although by now the mechanisms for doing so are well understood (see, for example, Hilbert [22, Chapter IV]). We are confident that the methods described here extend straightforwardly to cover areas as well, but spelling out the details would require more effort.

\subsection{Tarski's system}

Tarski's axiomatization of the ruler-and-compass fragment of Euclidean geometry employs the language, $\mathcal{L}$, whose only nonlogical predicates are a ternary predicate, $B$, where $B(a b c)$ is intended to denote that $a, b$, and $c$ are collinear and $b$ is between $a$ and $c$; and a four-place relation, $\equiv$, where $a b \equiv c d$ is intended to denote that segment $a b$ is congruent to segment $c d$. (In contrast to the "between" predicate of $E$, Tarski's $B$ denotes nonstrict betweenness.) The axioms consist of (the universal closures of) the following (see, e.g. [58]):

1. Equidistance axiom (E1): $a b \equiv b a$

2. Equidistance axiom (E2): $(a b \equiv p q) \wedge(a b \equiv r s) \rightarrow(p q \equiv r s)$

3. Equidistance axiom (E3): $(a b \equiv c c) \rightarrow a=b$

4. Betweenness axiom (B): $B(a b d) \wedge B(b c d) \rightarrow B(a b c)$

5. Segment Construction Axiom (SC): $\exists x(B(q a x) \wedge(a x \equiv b c))$

6. Five-Segment Axiom (5S):

$$
\begin{aligned}
{[\neg(a=b) \wedge B(a b c)} & \wedge B(p q r) \\
(a d \equiv p s) & \wedge(b d \equiv p q \equiv q s)] \rightarrow(c d \equiv r s)
\end{aligned}
$$

7. Pasch Axiom $(\mathrm{P}): B(a p c) \wedge B(q c b) \rightarrow \exists x(B(a x q) \wedge B(b p x))$

8. Lower 2-Dimension Axiom (2L): $\exists a, b, c[\neg B(a b c) \wedge \neg B(b c a) \wedge \neg B(c a b)]$

9. Upper 2-Dimension Axiom (2U): $\neg(a=b) \wedge \bigwedge_{i=1}^{3} x_{i} a \equiv x_{i} b \rightarrow\left(B\left(x_{1} x_{2} x_{3}\right) \vee\right.$ $\left.B\left(x_{2} x_{3} x_{1}\right) \vee B\left(x_{3} x_{1} x_{2}\right)\right)$ 
10. Parallel Postulate (PP): $B(a d t) \wedge B(b d c) \wedge \neg(a=d) \rightarrow \exists x, y(B(a b x) \wedge$ $B(a c y) \wedge B(y t x))$

11. Intersection Axiom (Int): $\left(a x \equiv a x^{\prime}\right) \wedge\left(a z \equiv a z^{\prime}\right) \wedge B(a x z) \wedge B(x y z) \rightarrow$ $\exists y^{\prime}\left(\left(a y \equiv a y^{\prime}\right) \wedge B\left(x^{\prime} y^{\prime} z^{\prime}\right)\right)$

Intuitively, the last axiom says that any line through a point lying inside a circle intersects the circle. Tarski showed that when one replaces this axiom with the Continuity Axiom Scheme,

$$
\exists a \forall x, y(\varphi(x) \wedge \psi(y) \rightarrow B(a x y)) \rightarrow \exists b \forall x, y(\varphi(x) \wedge \psi(y) \rightarrow B(x b y))
$$

the result is complete for the semantics of the full Euclidean plane. But he also showed that axioms 1-11 are complete for ruler-and-compass constructions, and it is this result that is important for our purposes 10

Theorem 5.2 (Tarski). If $\varphi$ is valid for ruler-and-compass constructions, then $\varphi$ is a first-order consequence of the axioms above.

We will now fashion a variant of this system with better proof-theoretic properties. A theory is called geometric if all of its axioms are sentences of the following form:

$$
(\star) \quad \forall \vec{x}\left[\bigwedge_{i=1}^{m} B_{i}(\vec{x}) \rightarrow \bigvee_{j=1}^{n}\left(\exists \vec{y}_{j} \bigwedge_{k=1}^{\ell_{j}} A_{j, k}\left(\vec{x}, \vec{y}_{j}\right)\right)\right],
$$

where the $A$ 's and $B$ 's are atomic formulas (including $\top$ and $\perp$ ), and each of $\vec{x}$, $\vec{y}$ or the antecedent of the conditional could be empty. Formulas of the form $(\star)$ are called geometric. Those geometric formulas with only a single disjunct in the consequent (i.e. geometric formulas in which $\vee$ does not appear) are called regular. Note that, on our modeling, Euclid's propositions are almost of this latter form, the difference being that arbitrary literals (negated atomic formulas as well as atomic formulas) are allowed in the antecedent and consequent.

Sara Negri 42, building on earlier joint work with Jan von Plato 43, has established a cut-elimination theorem for geometric theories that we can put to use in our completeness proof. Suppose we have a geometric theory formulated in a standard two-sided sequent calculus (see, for example [7, 59]). Then the theory can be recast equivalently by replacing each of its geometric axioms like the one above with a corresponding inference rule, called a geometric rule scheme (GRS):

\footnotetext{
${ }^{10}$ Note that the system for ruler-and-compass constructions is finitely axiomatized, in contrast to the stronger system with the Continuity Axiom Scheme. Ziegler [66] proved that any finitely axiomatizable theory of fields that has among its models an algebraically closed field, a real closed field or a field of $p$-adic numbers, is an undecidable theory. It is clear from the present result that the formal system for ruler-and-compass constructions has a real closed field among its models (since a real closed field is, a fortiori, Euclidean). Thus the system is undecidable.
} 


$$
\frac{\vec{A}_{1, \cdot} \cdot\left(\vec{x}, \vec{y}_{1}\right), \Pi \Rightarrow \Theta \quad \cdots \quad \vec{A}_{n, \cdot}\left(\vec{x}, \vec{y}_{n}\right), \Pi \Rightarrow \Theta}{\vec{B}(\vec{x}), \Pi \Rightarrow \Theta}
$$

Here we assume that the variables among the $\vec{y}_{j}$ 's do not appear free in $\vec{B}, \Pi$ or $\Theta 11$ Negri's principal result is the following theorem, whose corollary we will apply later.

Theorem 5.3. Any sequent provable in a sequent calculus with geometric rule schemes has a cut-free proof.

Since the cut rule is the only rule that removes formulas, this shows that if a sequent $\Pi \Rightarrow \Theta$ is provable in such a system, there is a proof that mentions only subformulas of formulas in $\Pi$ and $\Theta$, and possibly some other atomic formulas.

Say a sequent $\Pi \Rightarrow \Theta$ is geometric if $\Pi$ is a set of atomic formulas and $\Theta$ is a finite set of existentially quantified conjunctions of atomic formulas. In other words, a geometric sequent is a representation of a geometric formula where the implication is replaced by the sequent arrow and the outer universal quantifiers are left implicit. Say a geometric sequent is regular if $\Theta$ consists of at most one formula. Theorem 5.3 implies that if we are working in a sequent calculus with geometric rule schemes, then any provable geometric sequent has a proof in which every sequent is geometric; and, similarly, any provable regular sequent has a proof in which every sequent is regular.

Tarski's axiomatization for the ruler-and-compass constructions is nearly geometric. The only stumbling block is that in $(\star)$ the conjunctions are required to be conjunctions of atomic formulas, not literals. Thus, for instance, the lower 2-dimensional axiom

$$
\exists a, b, c(\neg B(a b c) \wedge \neg B(b c a) \wedge \neg B(c a b))
$$

is not geometric. We remedy this situation by introducing explicit predicates for the negations of $=$ and $B$ and $\equiv$; that is, we expand our language to one called $\mathcal{L}(T)$ by adding predicates $\neq$ and $\bar{B}$ and $\not \equiv$; and we add the (geometric) axioms

- $\forall x, y((x=y) \vee(x \neq y))$

- $\forall x, y((x=y) \wedge(x \neq y) \rightarrow \perp)$

as well as analogous ones for $B, \bar{B}$ and $\equiv, \not \equiv$. We will call these "negativity axioms" below. Also, we replace any negated instances of $=$ or $B$ (there are no such negated instances of $\equiv$ ) from Tarski's original axiomatization with the new corresponding predicate, thus obtaining a geometrically axiomatized theory.

Notice that there is an obvious translation from the language $\mathcal{L}(T)$ of $T$ to the language of Tarski's system, which maps, e.g., occurrences of $\bar{B}(x y z)$ to

\footnotetext{
${ }^{11}$ If one represents sequents using sequences or multisets of formulas, as Negri does, the rules must be presented with the $\vec{B}(\vec{x})$ repeated in the premises in order for Negri to prove the admissibility of the structural rules of contraction and weakening, along with cut-elimination. Taking $\Pi$ and $\Theta$ to be sets is notationally simpler and suffices for our purposes.
} 
$\neg B(x y z)$, and so on. This translation preserves provability, since the negativity axioms imply that the new predicates behave like negations. We now go further and put the nonlogical axioms of $T$ into the form of geometric rule schemes. First of all, the negativity axioms look like this:

$$
\begin{gathered}
\frac{(x=y), \Pi \Rightarrow \Theta \quad(x \neq y), \Pi \Rightarrow \Theta}{\Pi \Rightarrow \Theta} \mathrm{Neg} \\
\frac{\perp, \Pi \Rightarrow \Theta}{(x=y),(x \neq y), \Pi \Rightarrow \Theta} \mathrm{Neg}
\end{gathered}
$$

and similarly for the other predicates. The remaining rules are as follows (and note that variables appearing in parentheses next to the rule names are those which are not allowed to appear free in the conclusion):

$$
\begin{gathered}
\frac{a b \equiv b a, \Pi \Rightarrow \Theta}{\Pi \Rightarrow \Theta} \mathrm{E} 1 \\
\frac{(p q \equiv r s), \Pi \Rightarrow \Theta}{(a b \equiv p q),(a b \equiv r s), \Pi \Rightarrow \Theta} \mathrm{E} 2 \\
\frac{(a=b), \Pi \Rightarrow \Theta}{(a b \equiv c c), \Pi \Rightarrow \Theta} \mathrm{E} 3 \\
\frac{B(a b c), \Pi \Rightarrow \Theta}{B(a b d), B(b c d), \Pi \Rightarrow \Theta} \mathrm{B} \\
\frac{B(q a x),(a x \equiv b c), \Pi \Rightarrow \Theta}{\Pi \Rightarrow \Theta} \mathrm{SC}(\mathrm{x}) \\
\frac{B \neq(a x q), B(b p x), \Pi \Rightarrow \Theta}{B(a p c), B(q c b), \Pi \Rightarrow \Theta} \mathrm{P}(\mathrm{x}) \\
\frac{\bar{B}(a b c), \bar{B}(b c a), \bar{B}(c a b), \Pi \Rightarrow \Theta}{\Pi \Rightarrow \Theta} 2 \mathrm{~L}(\mathrm{a}, \mathrm{b}, \mathrm{c}) \\
\frac{B(a b x), B(a c y), B(y t x), \Pi \Rightarrow \Theta}{B(a d t), B(b d c), a \neq d, \Pi \Rightarrow \Theta} \mathrm{PP}(\mathrm{x}, \mathrm{y}) \\
B\left(x_{1} x_{2} x_{3}\right), \Pi \Rightarrow \Theta\left(x_{2} x_{3} x_{1}\right), \Pi \Rightarrow \Theta \\
a \neq b,\left(x_{1} a \equiv x_{1} b\right),\left(x_{2} a \equiv x_{2} b\right),\left(x_{3} a \equiv x_{3} b\right), \Pi \Rightarrow \Theta
\end{gathered}
$$




$$
\frac{\left(a y \equiv a y^{\prime}\right), B\left(x^{\prime} y^{\prime} z^{\prime}\right), \Pi \Rightarrow \Theta}{\left(a x \equiv a x^{\prime}\right),\left(a z \equiv a z^{\prime}\right), B(a x z), B(x y z), \Pi \Rightarrow \Theta} \operatorname{Int}\left(y^{\prime}\right)
$$

Since the resulting system is just a reworking of Tarski's axiomatization, combining Theorem 5.2 with Negri's Theorem 5.3 yields the following:

Lemma 5.4. Let $\Pi \Rightarrow \Theta$ be a geometric sequent in the language of $T$ that is valid for ruler-and-compass constructions. Then $\Pi \Rightarrow \Theta$ has a cut-free proof in $T$.

\subsection{Translating $E$ to $T$}

Our goal now is to provide a translation $\pi$ that maps any sequent $\Gamma \Rightarrow \exists \vec{x} . \Delta$ of $E$ to a geometric (in fact, regular) sequent $\Pi \Rightarrow \Theta$ of $T$, with the following properties:

- The translation preserves ruler-and-compass semantics, so that if $\Gamma \Rightarrow$ $\exists \vec{x}$. $\Delta$ is valid for ruler-and-compass constructions, so is $\Pi \Rightarrow \Theta$.

- Conversely, the existence of a cut-free proof of $\Pi \Rightarrow \Theta$ in $T$ implies the existence of a proof of $\Gamma \Rightarrow \exists \vec{x} . \Delta$ in $E$.

In this section we will define the translation and show that it satisfies the first property. The second property is then established in Section 5.4 below.

In carrying out the translation, we will represent each line $L$ of $E$ by distinct points $\mathfrak{c}_{1}^{L}, \mathfrak{c}_{2}^{L}$ that are assumed to lie on $L$. Similarly, we will represent each circle $\gamma$ of $E$ by its center, $\mathfrak{c}_{1}^{\gamma}$, and a point, $\mathfrak{c}_{2}^{\gamma}$, that is assumed to lie on $\gamma$. More precisely, given any sequent $\Gamma \Rightarrow \exists \vec{x}$. $\Delta$ of $E$, we will choose fresh variables $\mathfrak{c}_{1}^{L}, \mathfrak{c}_{2}^{L}$ for each line variable $L$ occurring in the sequent, and fresh variables $\mathfrak{c}_{1}^{\gamma}, \mathfrak{c}_{2}^{\gamma}$ for each circle variable $\gamma$. Let $\hat{\Delta}$ consist of the assumptions

$$
\left\{\mathfrak{c}_{1}^{L} \neq \mathfrak{c}_{2}^{L}, \text { on }\left(\mathfrak{c}_{1}^{L}, L\right), \text { on }\left(\mathfrak{c}_{2}^{L}, L\right)\right\}
$$

for each line variable $L$ among $\vec{x}$, and the assumptions

$$
\left\{\operatorname{center}\left(\mathfrak{c}_{1}^{\gamma}, \gamma\right), \operatorname{on}\left(\mathfrak{c}_{2}^{\gamma}, \gamma\right)\right\}
$$

for each circle variable $\gamma$ among $\vec{x}$. (Note that, in $E, \mathfrak{c}_{1}^{\gamma} \neq \mathfrak{c}_{2}^{\gamma}$ is a consequence of the latter set of assertions.) Let $\hat{\Gamma}$ consist of the assumptions corresponding to the remaining line and circle variables in the sequent. Then clearly $\Gamma \Rightarrow \exists \vec{x} . \Delta$ is provable in $E$ if and only if $\Gamma, \hat{\Gamma} \Rightarrow \exists \vec{x}, \vec{c} . \Delta, \hat{\Delta}$ is; and one is valid if and only if the other is valid as well. When we translate $\Gamma \Rightarrow \exists \vec{x}$. $\Delta$ to the language of $T$, we will use these new variables, and the translations will make sense as long as we assume $\mathfrak{c}_{1}^{L} \neq \mathfrak{c}_{2}^{L}$ and $\mathfrak{c}_{1}^{\gamma} \neq \mathfrak{c}_{2}^{\gamma}$ for the relevant constants. When we translate back, we will add the assumptions in $\hat{\Gamma}, \hat{\Delta}$, which will make it possible for $E$ to show that the result is equivalent to the original sequent. 


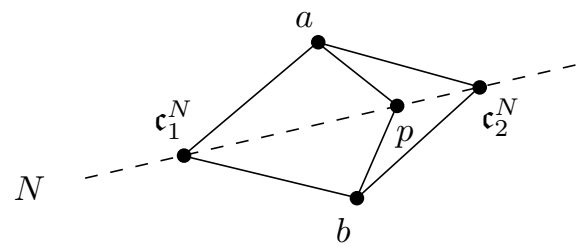

Figure 10: The translation of on $(p, N)$

To define $\pi$, first, for each $E$-literal $A$ we will define a corresponding $\mathcal{L}(T)$ formula $\bar{\pi}(A)$ of the following form:

$$
\exists \vec{z}\left(\bigwedge_{k} M_{k}(\vec{z})\right)
$$

where the $M_{k}$ 's are atomic. (Formulas of this form are sometimes referred to as positive primitive formulas.) We will occasionally abuse notation below and write $\bar{\pi}(A)$ for the conjunction $\bigwedge_{k} M_{k}(\vec{z})$ without the existential quantifiers out front. Furthermore, if we have a set of literals $A_{1}, \ldots, A_{m}$ and

$$
\bar{\pi}\left(A_{i}\right)=\exists \vec{z}_{i}\left(\bigwedge_{k=1}^{n_{i}} M_{i, k}\left(\vec{z}_{i}\right)\right)
$$

for each $i$, we will sometimes write $\bar{\pi}\left(A_{1}, \ldots, A_{m}\right)$ to refer to

$$
\exists \vec{z}_{1}, \ldots, \vec{z}_{m} \bigwedge_{i=1}^{m} \bigwedge_{k=1}^{n_{i}} M_{i, k}\left(\vec{z}_{i}\right)
$$

We do so for the sake of perspicuity and simple readability. When making such abuses, we will call attention to the fact that we are doing so, and no confusion should arise.

In each case, our translation provides a natural way of expressing the corresponding literal of $E$ as a formula of the desired form, though some thought (and a diagram) is often needed to make sense of it. For example, the translation of on $(p, N)$ is illustrated by Figure 10, For the diagrammatic assertions, the clauses of the translation are as follows.

$$
\begin{aligned}
\text { - } \operatorname{on}(p, N) \mapsto \exists a, b(\underbrace{a \neq b \wedge \mathfrak{c}_{1}^{N} a \equiv \mathfrak{c}_{1}^{N} b \wedge \mathfrak{c}_{2}^{N} a \equiv \mathfrak{c}_{2}^{N} b \wedge p a \equiv p b}_{=: \zeta\left(\mathfrak{c}_{1}^{N}, \mathfrak{c}_{2}^{N}, p, a, b\right)}) . \\
\text { - } \neg \mathrm{on}(p, N) \mapsto \underbrace{\bar{B}\left(\mathfrak{c}_{1}^{N} \mathfrak{c}_{2}^{N} p\right) \wedge \bar{B}\left(\mathfrak{c}_{1}^{N} p \mathfrak{c}_{2}^{N}\right) \wedge \bar{B}\left(p \mathfrak{c}_{1}^{N} \mathfrak{c}_{2}^{N}\right)}_{=: \chi\left(\mathfrak{c}_{1}^{N}, \mathfrak{c}_{2}^{N}, p\right)} .
\end{aligned}
$$

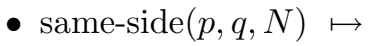

$$
\exists r, s, t, a, b\left(\zeta\left(\mathfrak{c}_{1}^{N}, \mathfrak{c}_{2}^{N}, s, a, b\right) \wedge \zeta\left(\mathfrak{c}_{1}^{N}, \mathfrak{c}_{2}^{N}, t, a, b\right) \wedge \chi\left(\mathfrak{c}_{1}^{N}, \mathfrak{c}_{2}^{N}, r\right) \wedge B(p r s) \wedge B(q r t)\right) .
$$


- $\neg$ same-side $(p, q, N) \mapsto \exists r, a, b\left(\zeta\left(\mathfrak{c}_{1}^{N}, \mathfrak{c}_{2}^{N}, r, a, b\right) \wedge B(p r q)\right)$.

- $\operatorname{between}(p, q, r) \mapsto B(p q r) \wedge p \neq q \wedge q \neq r \wedge p \neq r$.

- $\neg$ between $(p, q, r) \mapsto$

$$
\exists a, b, f, g, h, x, y, z\left[\begin{array}{l}
\chi(a, b, q) \wedge a \neq p \wedge a \neq q \wedge a \neq r \wedge b \neq p \wedge b \neq q \wedge b \neq r \wedge \\
B(a p x) \wedge B(a q y) \wedge B(\text { arz }) \wedge p \neq x \wedge q \neq y \wedge r \neq z \wedge \\
B(b p f) \wedge B(b q g) \wedge B(b r h) \wedge p \neq f \wedge q \neq g \wedge r \neq h \wedge \\
\bar{B}(x y z) \wedge \bar{B}(\text { fgh })
\end{array}\right]
$$

- on $(p, \gamma) \mapsto \mathfrak{c}_{1}^{\gamma} p \equiv \mathfrak{c}_{1}^{\gamma} \mathfrak{c}_{2}^{\gamma}$.

- $\neg \mathrm{on}(p, \gamma) \mapsto \mathfrak{c}_{1}^{\gamma} p \not \equiv \mathfrak{c}_{1}^{\gamma} \mathfrak{c}_{2}^{\gamma}$.

- inside $(p, \gamma) \mapsto \exists x\left(B\left(\mathfrak{c}_{1}^{\gamma} p x\right) \wedge p \neq x \wedge\left(\mathfrak{c}_{1}^{\gamma} x \equiv \mathfrak{c}_{1}^{\gamma} \mathfrak{c}_{2}^{\gamma}\right)\right)$.

- $\neg$ inside $(p, \gamma) \mapsto \exists x\left(B\left(\mathfrak{c}_{1}^{\gamma} x p\right) \wedge\left(\mathfrak{c}_{1}^{\gamma} x \equiv \mathfrak{c}_{1}^{\gamma} \mathfrak{c}_{2}^{\gamma}\right)\right)$.

These can be used to define equality and disequality for lines and circles:

- $L=M \mapsto \operatorname{on}\left(\mathfrak{c}_{1}^{L}, M\right) \wedge$ on $\left(\mathfrak{c}_{2}^{L}, M\right)$.

- $L \neq M \mapsto \exists x(\operatorname{on}(x, L) \wedge \neg \mathrm{on}(x, M))$.

- $\gamma=\delta \mapsto \mathfrak{c}_{1}^{\gamma}=\mathfrak{c}_{1}^{\delta} \wedge \mathfrak{c}_{1}^{\gamma} \mathfrak{c}_{2}^{\gamma} \equiv \mathfrak{c}_{1}^{\delta} \mathfrak{c}_{2}^{\delta}$

- $\gamma \neq \delta \mapsto \exists x(\mathrm{on}(x, \gamma) \wedge \neg \mathrm{on}(x, \delta))$.

More precisely, the translation involves expanding the $\bar{\pi}$ images of the literals on the right-hand side, and bringing the existential quantifiers to the front.

We have not yet indicated the $\bar{\pi}$-images for literals involving the intersects predicate. The positive literals are straightforwardly expressed in terms of literals that have already been translated:

- intersects $(L, M) \mapsto L \neq M \wedge \exists x(\operatorname{on}(x, L) \wedge$ on $(x, M))$.

- $\operatorname{intersects}(L, \gamma) \mapsto \exists x, y(x \neq y \wedge \mathrm{on}(x, L) \wedge \mathrm{on}(x, \gamma) \wedge \mathrm{on}(y, L) \wedge \mathrm{on}(y, \gamma))$.

- intersects $(\gamma, \delta) \mapsto \gamma \neq \delta \wedge \exists x, y(x \neq y \wedge$ on $(x, \gamma) \wedge \mathrm{on}(x, \delta) \wedge \mathrm{on}(y, \gamma) \wedge$ on $(y, \delta))$.

The negative literals, which assert nonintersection, require something more roundabout. For instance, we express the fact that $\alpha$ and $\beta$ do not intersect by saying that the line segment from the center of $\alpha$ to the center of $\beta$ encounters a point on $\alpha$ strictly before a point on $\beta$ :

$$
\neg \text { intersects }(\alpha, \beta) \mapsto \exists p, a, b\left[\begin{array}{l}
\mathfrak{c}_{1}^{\alpha} \mathfrak{c}_{2}^{\alpha} \equiv \mathfrak{c}_{1}^{\alpha} a \wedge \mathfrak{c}_{1}^{\beta} \mathfrak{c}_{2}^{\beta} \equiv \mathfrak{c}_{1}^{\beta} b \wedge a \neq b \wedge \\
B\left(\mathfrak{c}_{1}^{\alpha} a p\right) \wedge B\left(\mathfrak{c}_{1}^{\beta} b p\right) \wedge B(a p b)
\end{array}\right]
$$


Appropriate positive primitive $\bar{\pi}$-images for the literals $\neg \operatorname{intersects}(L, \alpha)$ and $\neg \operatorname{intersects}(L, M)$ can be found using $\bar{\pi}$-images from above, as well as the translation for $\angle x y z=$ right-angle which is given below. For instance, to say that $\neg$ intersects $(L, \alpha)$, we assert the existence of points $a, b, c$, where $a$ is on $\alpha, b \neq c$ are on $L, a$ is strictly between $\mathfrak{c}_{1}^{\alpha}$ and $b$, and $\angle a b c=$ right-angle. Similarly, $\neg$ intersects $(L, M)$ can be expressed by asserting the existence of $a, b, c, d$, where $a \neq b$ are on $L, c \neq d$ are on $M$, and the angles $\angle a b c$ and $\angle b c d$ are right angles.

The last type of literal to treat is that of metric assertions about segments and angles. Those for segments are more straightforward. Any term of the segment sort will be of the form $\overline{p_{1} q_{1}}+\cdots+\overline{p_{k} q_{k}}$ (we can ignore occurrences of 0 ; the translation below also makes sense for "empty sums"). Two such sums are equal if the segments can be laid side by side along a line so that the starting and ending points are the same. So, under our translation,

$$
\overline{p_{1} q_{1}}+\cdots+\overline{p_{k} q_{k}}=\overline{u_{1} v_{1}}+\cdots+\overline{u_{m} v_{m}}
$$

maps to

$$
\exists a_{0} \ldots a_{k}, b_{0} \ldots b_{m}\left[\begin{array}{l}
B\left(a_{0} a_{1} a_{2}\right), B\left(a_{1} a_{2} a_{3}\right), \ldots, B\left(a_{k-2} a_{k-1} a_{k}\right), \\
B\left(b_{0} b_{1} b_{2}\right), B\left(b_{1} b_{2} b_{3}\right), \ldots, B\left(b_{k-2} b_{k-1} b_{k}\right), \\
\left(p_{1} q_{1} \equiv a_{0} a_{1}\right),\left(p_{2} q_{2} \equiv a_{1} a_{2}\right), \ldots,\left(p_{k} q_{k} \equiv a_{k-1} a_{k}\right), \\
\left(u_{1} v_{1} \equiv b_{0} b_{1}\right),\left(u_{2} v_{2} \equiv b_{1} b_{2}\right), \ldots,\left(u_{m} v_{m} \equiv b_{m-1} b_{m}\right), \\
a_{0}=b_{0}, a_{k}=b_{m}
\end{array}\right]
$$

The translations of the other segment literals are obtained from this one with minor changes to the last part. Namely, the corresponding translations are obtained by making the following indicated changes to the last line above:

$$
\begin{aligned}
\sum_{i} \overline{p_{i} q_{i}} \neq \sum_{j} \overline{u_{j} v_{j}} & \mapsto a_{0}=b_{0}, a_{k} \neq b_{m} \\
\sum_{i} \overline{p_{i} q_{i}}<\sum_{j} \overline{u_{j} v_{j}} & \mapsto a_{0}=b_{0}, a_{k} \neq b_{m}, B\left(b_{0}, a_{k}, b_{m}\right) \\
\sum_{i} \overline{p_{i} q_{i}} \nless \sum_{j} \overline{u_{j} v_{j}} & \mapsto a_{0}=b_{0}, B\left(a_{0} b_{m} a_{k}\right)
\end{aligned}
$$

For the angle literals, a little care is needed. First, note that we can define equality and inequalities of angles as follows:

- $\angle x y z=\angle x^{\prime} y^{\prime} z^{\prime} \mapsto$

$$
\exists u, v, u^{\prime}, v^{\prime}(\underbrace{B(x u y) \wedge B(y v z) \wedge B\left(x^{\prime} u^{\prime} y^{\prime}\right) \wedge B\left(y^{\prime} v^{\prime} z^{\prime}\right) \wedge\left(u y \equiv u^{\prime} y^{\prime}\right) \wedge\left(y v \equiv y^{\prime} v^{\prime}\right)}_{=: \xi\left(x, y, z, x^{\prime}, y^{\prime}, z^{\prime}, u, v, u^{\prime}, v^{\prime}\right)} \wedge\left(u v \equiv u^{\prime} v^{\prime}\right)) .
$$

- $\neg\left(\angle x y z=\angle x^{\prime} y^{\prime} z^{\prime}\right) \mapsto$

$$
\exists u, v, u^{\prime}, v^{\prime}\left(\xi\left(x, y, z, x^{\prime}, y^{\prime}, z^{\prime}, u, v, u^{\prime}, v^{\prime}\right) \wedge\left(u v \not \equiv u^{\prime} v^{\prime}\right)\right) .
$$




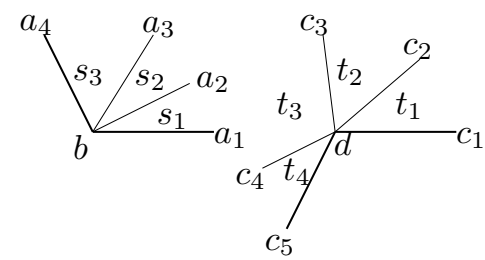

Figure 11: Here $\angle a_{1} b a_{4}=\angle c_{1} d c_{5}$, but $\sum s_{i}=2 \pi / 3$ while $\sum t_{i}=4 \pi / 3$.

- $\angle x y z<\angle x^{\prime} y^{\prime} z^{\prime} \mapsto$

$$
\exists u, v, u^{\prime}, v^{\prime}, a^{\prime}\left(\xi\left(x, y, z, x^{\prime}, y^{\prime}, z^{\prime}, u, v, u^{\prime}, v^{\prime}\right) \wedge a^{\prime} \neq v^{\prime} \wedge B\left(u^{\prime} a^{\prime} v^{\prime}\right) \wedge\left(u v \equiv u^{\prime} a^{\prime}\right)\right)
$$

- $\neg\left(\angle x y z<\angle x^{\prime} y^{\prime} z^{\prime}\right) \mapsto$

$$
\exists u, v, u^{\prime}, v^{\prime}, a\left(\xi\left(x, y, z, x^{\prime}, y^{\prime}, z^{\prime}, u, v, u^{\prime}, v^{\prime}\right) \wedge B(u a v) \wedge\left(u a \equiv u^{\prime} v^{\prime}\right)\right)
$$

We can also say that an angle is a right angle:

- $\angle x y z=$ right-angle $\mapsto$

$$
\exists p, u, v, u^{\prime}, v^{\prime}\left(x \neq y \wedge y \neq z \wedge p \neq y \wedge B(p y z) \wedge \xi\left(x, y, z, x, y, p, u, v, u^{\prime}, v^{\prime}\right) \wedge\left(u v \equiv u^{\prime} v^{\prime}\right)\right)
$$

At issue is how to compare sums of angles. Suppose we have two sums $\sum s_{i}$, $\sum t_{i}$ of angle terms. In analogy to the segment case, we would like to take the various angles in two given sums, reconstruct them by "stacking them up" via a series of points around respective fixed vertices, and then compare the sums by measuring the resulting angles formed by the initial and final points. The reason this can fail is that such a measure does not compare the sums themselves, but rather whether

$$
\begin{aligned}
\min \left\{\sum s_{i}(\bmod 2 \pi), 2 \pi-\right. & \left.\left(\sum s_{i}(\bmod 2 \pi)\right)\right\}= \\
& \min \left\{\sum t_{i}(\bmod 2 \pi), 2 \pi-\left(\sum t_{i}(\bmod 2 \pi)\right)\right\},
\end{aligned}
$$

so that unequal sums might be identified with one another. (See Figure 11 for instance.)

To remedy this, we do not stack the original angles. Instead, if comparing a $k$-fold sum and an $m$-fold sum, we let $n=\max (k, m)$ and compare $n$-fold bisections of the summand angles. The point is that the resulting angles are guaranteed to be no greater than the greatest of the original angles:

$$
\frac{1}{2^{n}} \sum_{i=1}^{n} \angle x_{i} y_{i} z_{i} \leq \frac{1}{2^{n}}\left(n \max _{i}\left\{\angle x_{i} y_{i} z_{i}\right\}\right) \leq \max \left\{\angle x_{i} y_{i} z_{i}\right\}
$$


Thus our choice of taking $\max (k, m)$-fold bisections means that our modified stacks all fit within one of the original angles from one of the sums, and $E$ 's setup guarantees that the term denotes an angle less than or equal to $\pi$. Thus we can make the kind of straightforward comparison of these shrunken stacks that we would like.

Given that longwinded explanation, we will not spell out the translation of the angle literals in detail, and will only briefly indicate how one of them proceeds; the others result from minor modifications of it, as with other groups of literals above. First we want an auxiliary $T$-formula which says " $\angle p^{\prime} q^{\prime} r^{\prime}=$ $\left(1 / 2^{n}\right) \angle p q r$," i.e. that the former is an $n$-fold bisection of the latter. The following works:

$$
\exists a, b, a^{\prime}, b^{\prime}, u_{1}, \ldots, u_{n}\left[\begin{array}{l}
B(q a p), B(q b r), B\left(q^{\prime} a^{\prime} p^{\prime}\right), B\left(q^{\prime} b^{\prime} r^{\prime}\right), \\
B\left(a u_{1} u_{2}\right), B\left(u_{1} u_{2} u_{3}\right), \ldots, B\left(u_{n-2} u_{n-1} u_{n}\right), B\left(u_{n-1} u_{n} b\right), \\
\left(\angle a^{\prime} q^{\prime} b^{\prime}=\angle u_{1} q u_{2}\right),\left(\angle a^{\prime} q^{\prime} b^{\prime}=\angle u_{2} q u_{3}\right), \ldots,\left(\angle a^{\prime} q^{\prime} b^{\prime}=\angle u_{n} q b\right), \\
\angle a q u_{1}=\angle u_{1} q b
\end{array}\right]
$$

The translation of the literal

$$
\sum_{i=1}^{k} \angle x_{i} y_{i} z_{i}=\sum_{j=1}^{m} p_{i} q_{i} r_{i}
$$

would then use the preceding formula, along with the formula $\xi$ from the translations of the diagrammatic angle literals above, in order to construct a positive primitive formula asserting the existence of two stackings of $\max (k, m)$-fold bisections of the original angles which, when compared in a similar fashion as the segment metric assertions were, are seen to be equal. The details are tedious to spell out, but straightforward.

We now extend $\pi$ to a translation $\pi: \mathcal{L}(E) \rightarrow \mathcal{L}(T)$ that maps every sequent $\Gamma \Rightarrow \exists \vec{x} . \Delta$ of $E$ to a regular sequent of $T$. Suppose $\Gamma \Rightarrow \exists \vec{x} . \Delta$ is of the form

$$
A_{1}, \ldots, A_{k} \Rightarrow \exists \vec{x} . B_{1}, \ldots, B_{m}
$$

where we have

$$
\bar{\pi}\left(A_{i}\right)=\exists \vec{z}_{i}\left(\bigwedge_{q=1}^{n_{i}} M_{i, q}\right), \quad \bar{\pi}\left(B_{j}\right)=\exists \vec{y}_{j}\left(\bigwedge_{r=1}^{p_{j}} N_{j, r}\right) .
$$

Let $\Delta^{\prime}$ consist of the assumption $\mathfrak{c}_{1}^{L} \neq \mathfrak{c}_{2}^{L}$ for each line variable $L$ among $\vec{x}$, and the assumption $\mathfrak{c}_{1}^{\gamma} \neq \mathfrak{c}_{2}^{\gamma}$ for each circle variable $\gamma$ among $\vec{x}$. Let $\Gamma^{\prime}$ consist of the corresponding assumptions for the remaining line and circle variables in the sequent. We define the image of this sequent, under $\pi$, to be the regular sequent

$\Gamma^{\prime}, M_{1,1}, \ldots, M_{1, q_{1}}, \ldots M_{k, 1}, \ldots, M_{k, q_{k}} \Rightarrow \exists \vec{x}, \vec{y}_{1}, \ldots, \vec{y}_{m}, \overrightarrow{\mathfrak{c}} \bigwedge \Delta^{\prime} \wedge \bigwedge_{i=1}^{m}\left(\bigwedge_{r=1}^{p_{i}} N_{i, r}\right) 12$

${ }^{12}$ So, with our abuse of notation mentioned above, we could render this simply as

$$
\Gamma^{\prime}, \bar{\pi}\left(A_{1}\right), \ldots, \bar{\pi}\left(A_{k}\right) \Rightarrow \exists \vec{x}, \vec{y}_{1}, \ldots, \vec{y}_{m} \bigwedge \Delta^{\prime} \wedge \bigwedge_{i=1}^{m} \bar{\pi}\left(B_{i}\right)
$$


The following lemma captures all that we need to know about $\pi$.

Lemma 5.5. $\Gamma \Rightarrow \exists \vec{x} . \Delta$ is valid for ruler-and-compass constructions if and only if $\pi(\Gamma \Rightarrow \exists \vec{x} . \Delta)$ is.

Once we have crafted $\pi$ appropriately, the lemma is quite straightforward to prove, given a precise articulation of the cartesian interpretation of $\mathcal{L}(E)$ and $\mathcal{L}(T)$ in the plane built on any Euclidean field. Given the definition of $\pi$ in terms of $\bar{\pi}$, it suffices to prove the result for sequents consisting of a single literal; you can check that, for instance, the Technical Propositions in Section 4.5 prove the $\Rightarrow$ same-side $(p, q, L)$ case (given the soundness of $E$ ). Further details can be found in [14.

\subsection{Interpreting $T$ in $E$}

By Lemma [5.5, we know that if a sequent $\Gamma \Rightarrow \exists \vec{x}$. $\Delta$ in the language of $E$ is valid for ruler-and-compass constructions, then so is $\pi(\Gamma \Rightarrow \exists \vec{x} . \Delta)$. By Lemma 5.4, this implies that $\pi(\Gamma \Rightarrow \exists \vec{x}$. $\Delta)$ has a cut-free proof in $T$. All that remains is to define a mapping $\rho$ from regular sequents in the language of $T$ to sequents in the language of $E$, and show the following:

- If there is a cut-free proof of $\pi(\Gamma \Rightarrow \exists \vec{x}$. $\Delta)$ in $T$, then there is a proof of $\rho(\pi(\Gamma \Rightarrow \exists \vec{x} . \Delta))$ in $E$.

- If there is a proof of $\rho(\pi(\Gamma \Rightarrow \exists \vec{x} . \Delta))$ in $E$, there is a proof of $\Gamma \Rightarrow \exists \vec{x}$. $\Delta$ in $E$.

Once again, we first define a translation $\bar{\rho}$ for individual atomic formulas, and then extend the map to sequents. (And we will make the same abuse of notation below regarding $\rho$ as was noted for $\pi$.) The atomic formulas are mapped as follows:

$$
\begin{aligned}
& B(p q r) \quad \mapsto \quad(\exists L, a, b) .[a \neq b, a \neq p, a \neq q, a \neq r, b \neq p, b \neq q, b \neq r, \\
& \text { on }(a, L) \text {, on }(b, L) \text {, on }(p, L) \text {, on }(q, L) \text {, on }(r, L) \text {, between }(a, q, b) \text {, } \\
& \neg \operatorname{between}(a, q, p), \neg \operatorname{between}(p, a, q), \neg \operatorname{between}(q, b, r) \text {, } \\
& \neg \text { between }(r, q, b)] \\
& \bar{B}(p q r) \quad \mapsto \quad \neg \text { between }(p, q, r), p \neq q, q \neq r \\
& p=q \quad \mapsto \quad p=q \\
& p \neq q \quad \mapsto \quad \neg(p=q) \\
& x y \equiv v u \quad \mapsto \quad \overline{x y}=\frac{q}{v u} \\
& x y \not \equiv v u \quad \mapsto \quad \overline{x y} \neq \overline{v u}
\end{aligned}
$$

Why the first two are appropriate should be clear upon reflection (remembering that between $(p, q, r)$ is meant to be strict, while $B(p q r)$ is not), and the others are obvious.

We now extend the map to sequents

$$
P_{1}(\vec{x}), \ldots, P_{n}(\vec{x}) \Rightarrow \exists \vec{y}\left(\bigwedge_{j=1}^{l} Q_{j}(\vec{x}, \vec{y})\right) .
$$


Assuming each $P_{i}(\vec{x})$ is mapped to $\exists \vec{z}_{i} . M_{i}\left(\vec{x}, \vec{z}_{i}\right)$ by $\bar{\rho}$, where each $M_{i}$ is a set of literals, and assuming each $Q_{j}(\vec{x}, \vec{y})$ is mapped to $\exists \vec{w}_{i} . N_{j}\left(\vec{x}, \vec{y}, \vec{z}_{j}\right)$, the sequent above is mapped to the sequent

$$
M_{1}\left(\vec{x}, \vec{z}_{1}\right), \ldots, M_{k}\left(\vec{x}, \vec{z}_{k}\right) \Rightarrow \exists \vec{y}, \vec{w}_{1}, \ldots, \vec{w}_{l} . N_{1}\left(\vec{x}, \vec{y}, \vec{z}_{1}\right), \ldots, N_{l}\left(\vec{x}, \vec{y}, \vec{z}_{l}\right)
$$

of $E$ 13

We now proceed to establish the two properties indicated above. The next lemma establishes the first.

Lemma 5.6. If there is a cut-free proof of the regular sequent

$$
P_{1}(\vec{x}), \ldots, P_{n}(\vec{x}) \Rightarrow \exists \vec{y}\left(\bigwedge_{j} Q_{j}(\vec{x}, \vec{y})\right)
$$

in $T$, then there is a cut-free proof of its $\rho$ translation,

$$
M_{1}\left(\vec{x}, \vec{z}_{1}\right), \ldots, M_{k}\left(\vec{x}, \vec{z}_{k}\right) \Rightarrow \exists \vec{y}, \vec{w}_{1}, \ldots, \vec{w}_{l} . N_{1}\left(\vec{x}, \vec{y}, \vec{z}_{1}\right), \ldots, N_{l}\left(\vec{x}, \vec{y}, \vec{z}_{l}\right),
$$

in $E$.

Proof. We proceed by induction on the proof in $T$. We need to show that every inference of $T$ is mirrored by a proof in $E$. The logical axioms and the logical rules which can appear in a cut-free proof of a regular sequent are already incorporated into the machinery of $E$ :

- (Left/right conjunction rules). We note that we do not have the symbol $\wedge$ in the language of $E$; instances of it get unpacked via the translation $\rho$. The left rules becomes vacuous, and the right rule is easily checked to be a derived rule of $E$ (as an instance of theorem application).

- (Right exists rule). Similarly, uses of this rule disappear in the translation.

- (Left falsum rules). The effects of these rules are subsumed under E's notion of direct consequence.

- (Negativity axioms). Similarly straightforward.

We are left with the remaining GRS's from Section 5.2. With one exception, these are of the form

$$
\frac{A_{1}, \ldots, A_{n}, \Pi \Rightarrow \Theta}{B_{1}, \ldots, B_{m}, \Pi \Rightarrow \Theta}
$$

\footnotetext{
${ }^{13}$ Again, with abuse of notation this is just

$$
\bar{\rho}\left(P_{1}\right), \ldots, \bar{\rho}\left(P_{n}\right) \Rightarrow \exists \vec{y}, \vec{w}_{1}, \ldots, \vec{w}_{l} \cdot \bar{\rho}\left(Q_{1}\right), \ldots, \bar{\rho}\left(Q_{l}\right) .
$$
}


which is to say, they correspond to the Tarskian axioms which are regular. In these cases, it suffices by the induction hypothesis to show that $E$ proves

$$
\bar{\rho}\left(B_{1}\right), \ldots, \bar{\rho}\left(B_{m}\right) \Rightarrow \exists \vec{x} \cdot \bar{\rho}\left(A_{1}\right), \ldots, \bar{\rho}\left(A_{n}\right) .
$$

Note that we are using the abuse of notation described in the last section. Checking the details of this for the various regular GRS's is pretty painless. For instance:

- (E1,E2, E3). Given the trivial nature of $\bar{\rho}$ for $\equiv$ statements, it is easy to see that these cases are handled by our metric rules.

- (2L). Let $a$ be a point. Construct a point $b \neq a$. Construct line $L$ through $a, b$. Construct a point $c$ that is not on $L$. Each of between $(a, b, c)$ or between $(b, a, c)$ or between $(a, c, b)$ leads to on $(c, L)$, hence a contradiction. Thus in $E$ we can conclude $\neg$ between for each. One can check the definitions of $2 \mathrm{~L}$ and $\bar{\rho}$ to see that we have done what is needed.

- (SC). The Technical Propositions in Section 4.5 provide the needed $E$ constructions here.

- We omit the remaining cases, some of which are slightly more involved, but none of which are interesting or enlightening.

All that remains is the sole GRS which is not regular, the upper two-dimensional axiom. The situation is not really all that different from the regular cases; what we have to show, given the inductive hypothesis, is only slightly different.

The following suffices. Suppose we have $a \neq b$, and $\overline{x_{i} a}=\overline{x_{i} b}$ for $i=1,2,3$. We need $E$ to prove that two instances of $\neg$ between $\left(x_{i}, x_{j}, x_{k}\right)$ hold. We reason by cases; $a$ la Euclid we present only the case in which all the $x_{i}$ are distinct, as the other cases are only easier.

For each $i$, construct circle $\gamma_{i}$ with center $x_{i}$, passing through $b$. Construct line $L$ through $a, b$. By Proposition I.12 (formalized in $E$ above), construct line $M$ perpendicular to $L$. It is then a direct consequence that each $x_{i}$ is on $M$.

Once again, we reason by cases, considering each parity for each possible between $\left(x_{i}, x_{j}, x_{k}\right)$; there are eight cases (omitting symmetry in the between arguments). In the four for which two positive between relations were to hold, $E$ derives a contradiction. In the other four cases, we have two negative instances, which is what we needed.

Given the previous lemma, we are almost home. We have shown that if $\Gamma \Rightarrow \exists \vec{x} . \Delta$ is a valid sequent of $E$, then there is a cut-free proof of $\pi(\Gamma \Rightarrow \exists \vec{x} . \Delta)$ in $T$, and hence a proof of $\rho(\pi(\Gamma \Rightarrow \exists \vec{x} . \Delta))$ in $E$. The trouble, of course, is that $\rho(\pi(\Gamma \Rightarrow \exists \vec{x} . \Delta))$ is not quite the same thing as $\Gamma \Rightarrow \exists \vec{x}$. $\Delta$. For one thing, the lines and circles in the original sequent have been replaced by pairs of points representing them; and the translated sequent will typically feature extra points and hypotheses in both the antecedent and consequent. The next two lemmas demonstrate that, from the $E$ proof of the translated proposition, we can in fact recover a proof of the original proposition, $\Gamma \Rightarrow \exists \vec{x} . \Delta$. 
Lemma 5.7. Let $M(\vec{x})$ be any literal of E. Suppose that

$$
\bar{\pi}(M)=\exists \vec{z} \bigwedge_{j=1}^{m} Q_{j}(\vec{x}, \vec{z}),
$$

and further that

$$
\bar{\rho}\left(Q_{j}\right)=\exists \vec{y}_{j} \cdot A_{j, 1}, \ldots, A_{j, n_{j}} .
$$

Let $\hat{\Theta}$ consist of the assumptions

$$
\left\{\mathfrak{c}_{1}^{L} \neq \mathfrak{c}_{2}^{L}, \text { on }\left(\mathfrak{c}_{1}^{L}, L\right), \text { on }\left(\mathfrak{c}_{2}^{L}, L\right)\right\}
$$

for each line variable $L$ in $M$, and the assumptions

$$
\left\{\operatorname{center}\left(\mathfrak{c}_{1}^{\gamma}, \gamma\right), \text { on }\left(\mathfrak{c}_{2}^{\gamma}, \gamma\right)\right\}
$$

for each circle variable $\gamma$ in $M$. Then $E$ proves both

(1) $\hat{\Theta}, M \Rightarrow \exists \vec{z}, \vec{y}_{1}, \ldots, \vec{y}_{m} . A_{1,1}, \ldots, A_{1, n_{1}}, \ldots, A_{m, 1}, \ldots, A_{m, n_{m}}$.

(2) $\hat{\Theta}, A_{1,1}, \ldots, A_{1, n_{1}}, \ldots, A_{m, 1}, \ldots, A_{m, n_{m}} \Rightarrow \exists \vec{x} . \hat{M}$,

where $\vec{x}$ are the line and circle variables in $M$. Moreover, $E$ proves all sequents of the form

$$
\mathfrak{c}_{1}^{L} \neq \mathfrak{c}_{2}^{L} \Rightarrow \exists L . \operatorname{on}\left(\mathfrak{c}_{1}^{L}, L\right), \text { on }\left(\mathfrak{c}_{2}^{L}, L\right),
$$

and

$$
\mathfrak{c}_{1}^{\gamma} \neq \mathfrak{c}_{2}^{\gamma} \Rightarrow \exists \gamma . \operatorname{center}\left(\mathfrak{c}_{1}^{\gamma}, \gamma\right), \text { on }\left(\mathfrak{c}_{2}^{\gamma}, \gamma\right) .
$$

Before getting to the proof, we note that clause (1) of the lemma just says that $E$ proves $\hat{\Theta}, M \Rightarrow \bar{\rho}(\bar{\pi}(M))$ for any literal. Moreover, with our abuse of notation we can render the second part more perspicuously as asserting that $E$ proves $\hat{\Theta}, \bar{\rho}(\bar{\pi}(M)) \Rightarrow M$.

Proof. The last two claims in the lemma are immediate, using the construction rules of $E$. For the first two claims, in order to avoid needless tedium, we indicate details for only a few cases (and also indicate how trivial some of the cases are).

- (between $(p, q, r))$. We need to show that between $(p, q, r)$ is inter-derivable with

$$
\begin{aligned}
& \exists L . \text { on }(p, L), \text { on }(q, L), \text { on }(r, L), \neg \operatorname{between}(p, r, q), \neg \operatorname{between}(q, p, r), \\
& \qquad p \neq q, q \neq r, p \neq r .
\end{aligned}
$$

Supposing the latter, we can conclude between $(p, q, r)$ from the sixth betweenness rule.

For the converse, suppose between $(p, q, r)$. A couple of applications of our first betweenness rule yield $\neg$ between $(q, p, r)$, $\neg$ between $(p, r, q)$ and the distinctness assertions. Construct line $L$ through $p, q ; r$ is on $L$ as well, by the sixth and second betweenness rules. 
- $($ on $(p, \gamma)$ or $\neg$ on $(p, \gamma))$. This is immediate from the diagram-segment transfer axioms.

- $(\overline{x y}=\overline{z w}$ or $\overline{x y} \neq \overline{z w})$. Similarly easy.

- $(\overline{x y}<\overline{z w})$. In this case we need to show that the literal is inter-derivable with

$$
\begin{aligned}
\exists a, L \cdot \operatorname{on}(z, L), \text { on }(a, L), & \text { on }(w, L), a \neq w, z \neq w, \\
& \neg \operatorname{between}(a, z, w), \neg \operatorname{between}(z, w, a), \overline{x y}=\overline{z a} .
\end{aligned}
$$

Suppose the latter. In case $z \neq a$, it follows that between $(z, a, w)$ (betweenness rule 6). Then $\overline{z a}+\overline{a w}=\overline{z w}$ (diagram-segment rule 1). As $a \neq w, \overline{a w}>0$ (first metric inference). By our linear arithmetic, then, $\overline{z w}>\overline{x y}$ as desired. In the case $z=a$, we have $\overline{x y}=\overline{z a}=0$ and $\overline{z w}=\overline{a w}$. As $a \neq w, \overline{a w}>0$, so again we have $\overline{z w}>\overline{x y}$.

Conversely, suppose $\overline{x y}<\overline{z w}$. So $\overline{z w}>0$, hence $z \neq w$. Construct line $L$ through $z$ and $w$. In case $x=y$, then $z$ itself will be our $a$. In case $x \neq y$, apply Proposition I.2 to get a $b$ such that $\overline{x y}=\overline{z b}$. Draw circle $\beta$ through $b$ centered at $z$. As $z$ is inside $\beta$ and on $L$, we know that $\beta$ and line $L$ intersect. Since $\overline{z b}=\overline{x y}<\overline{z w}$, we know that $w$ lies outside $\beta$. Thus we may take the intersection point $a$ of $\beta$ and $L$ such that between $(z, a, w)$ (by the fourth intersection construction rule). This is the $a$ we need.

- $(\overline{x y} \nless \overline{z w})$. Similar to the previous.

Lemma 5.8. If $\rho(\pi(\Gamma \Rightarrow \exists \vec{x}$. $\Delta))$ is provable in $E$, then so is $\Gamma \Rightarrow \exists \vec{x} . \Delta$.

Proof. Let $\hat{\Gamma}$ and $\hat{\Delta}$ be the sets of formulas described at the beginning of Section 5.3. Using our abuses of notation, our supposition is that $E$ proves

$$
\bar{\rho}(\bar{\pi}(\Gamma)) \Rightarrow \exists \vec{z} \cdot \bar{\rho}(\bar{\pi}(\Delta)) .
$$

Repeated application of clause (1) of Lemma 5.7 shows that $E$ proves

$$
\Gamma, \hat{\Gamma} \Rightarrow \exists \vec{u} . \bar{\rho}(\bar{\pi}(\Gamma)),
$$

where $\vec{u}$ are the new variables picked up in the translation. Using theorem application, $E$ proves

$$
\Gamma, \hat{\Gamma} \Rightarrow \exists \vec{z}, \vec{u} \cdot \bar{\rho}(\bar{\pi}(\Delta)) .
$$

The last part of Lemma 5.7 shows that $E$ proves

$$
\Gamma, \hat{\Gamma} \Rightarrow \exists \vec{z}, \vec{u}, \vec{v} . \hat{\Delta}, \bar{\rho}(\bar{\pi}(\Delta)),
$$

where $\vec{v}$ are the line and circle variables among $\vec{x}$, lost in the translation back and forth, and now restored. Clause (2) of Lemma 5.7 then shows that $E$ proves

$$
\Gamma, \hat{\Gamma} \Rightarrow \exists \vec{z}, \vec{u}, \vec{v} . \hat{\Delta}, \Delta .
$$


Since the all the variables $\vec{x}$ are among $\vec{z}, \vec{u}, \vec{v}$, the sequent

$$
\Gamma, \hat{\Gamma} \Rightarrow \exists \vec{x} . \Delta
$$

is subsumed by the previous one. Since $E$ can also prove $\Gamma \Rightarrow \exists \overrightarrow{\mathfrak{c}} . \hat{\Gamma}$ for the new point variables that occur in $\hat{\Gamma}$, it can prove

$$
\Gamma \Rightarrow \exists \vec{x} . \Delta,
$$

as required.

Putting everything together, we have the proof of the completeness theorem.

Proof of Theorem 5.1. Suppose that $\Gamma \Rightarrow \exists \vec{x} . \Delta$ is valid for ruler-and-compass constructions. By Lemma5.5 $\pi(\Gamma \Rightarrow \exists \vec{x} . \Delta)$ is a valid sequent in the language of $T$. By Lemma 5.4 there is a cut-free proof of that sequent in $T$. By Lemma 5.6, $\rho(\pi(\Gamma \Rightarrow \exists \vec{x} . \Delta))$ is provable in $E$. By Lemma $5.8, \Gamma \Rightarrow \exists \vec{x} . \Delta$ is provable in $E$, as required.

\section{Implementation}

In Section 3.8, we argued that the set of one-step inferences in $E$ is decidable, as one would expect from any formal system. But given the fact that we are trying to model the inferential structure of the Elements, there is the implicit claim that verifying such inferences is within our cognitive capabilities, at least at the scale of complexity found in the proofs in the Elements. "Cognitively feasible" does not always line up with "computationally feasible," and it is often quite challenging to get computers to emulate common visual tasks. But, of course, our case would be strengthened if we could show that our inferences are computationally feasible as well.

In fact, our analysis should make it possible to design a computational proof checker based on $E$ that takes, as input, proofs that look like the ones in the Elements, and verifies their correctness against the rules of the system. In this section, we describe some preliminary studies that suggest that general purpose tools in automated reasoning are sufficient for the task 14

In Section 3.8, we noted that any fact obtained by a direct diagram inference is contained in the set of first-order consequences of the set of our universal axioms and the set of literals constituting the diagram. Furthermore, there are no function symbols in the language. These types of problems are fairly easy for off-the-shelf theorem provers for first-order logic. We entered our betweenness, same-side, and Pasch axioms in the standard TPTP format ("Thousands

\footnotetext{
${ }^{14}$ As part of his MS thesis work at Carnegie Mellon, Benjamin Northrop has written code in Java that carries out diagrammatic inferences using an eager saturation method: whenever a new object is added to the diagram, the system closes the diagram under rules and derives all the atomic and negation atomic consequences. The system works on small examples, but gets bogged down with diagrams of moderate complexity. But this does not rule out the fact that more sophisticated representations of the diagrammatic data might render such an approach viable. See the discussion later in this section.
} 
of Problems for Theorem Provers,"), described a simple diagram with five lines and six points, and checked a number of consequences with the systems E [51] (no relation to our "E") and Spass [63]. The consequences were verified instantaneously.

There is also a class of systems called "satisfiability modulo theories" solvers, or SMT solvers for short, which combine decision procedures for provability of universal sentences modulo the combination of disjoint theories whose universal fragments are decidable [33. Such systems typically include very fast decision procedures for linear arithmetic (that is, the linear theory of the reals). This is particularly helpful to us, since our metric inferences are of this sort. Unfortunately, SMT solvers do not provide complete decision procedures for the set of consequences of arbitrary universal axioms, which is what is needed to verify our diagrammatic and transfer inferences. Nonetheless, some solvers, like Z3 [13] and CVC3 22 provide heuristic instantiation of quantifiers. The advantage to using such systems is that they can handle not just the diagrammatic inferences, but the metric and transfer inferences as well. We entered all our axioms in the standard SMT format, and tested it with the two systems just mentioned. The results were promising; most inferences were instantaneous, and only a few required more than a few seconds. The diagram, axioms, and test queries can be found online, at Avigad's home page.

The fact that SMT solvers can handle arbitrary quantifier-free logic, and the fact that one can incrementally add and retract statements from the database of asserted facts, suggests that SMT solvers can provide a complete back end to a proof checker for $E$. The proof checker then need only parse an input proof, assert the relevant facts to the SMT solver, and check the claimed consequences. More specifically, when the user asserts a theorem, the proof checker should declare the new objects (points, lines, and circles) to the SMT solver, assert the assumptions to the SMT solver, and store the conclusion. When the user enters a construction rule, the proof checker should check that the prerequisites are consequences of the facts already asserted to the SMT solver, create the new objects, and assert their properties. Applying a previously proved theorem is handled in a similar way. When a user enters "hence $A$," the proof checker should check that $A$ is a consequence of the facts already asserted to the SMT solver, and, if so, assert it explicitly to the SMT database, to facilitate subsequent inferences. To handle suppositional reasoning (that is, proof by contradiction, or a branch of a case split), the proof checker should "push" the state of the SMT database and temporarily assert the local hypothesis, and then, once the desired conclusion is verified, "pop" the state and assert the resulting conditional. Finally, when the user enters "Q.E.D." or "Q.E.F.", the proof checker need only check that the negation of the theorem's conclusion is inconsistent with the facts that have been asserted to the SMT solver.

Finally, we note that there has been recent work unifying resolution and SMT frameworks, for example, with the Spass+T system [48]. Such a system should be well-suited to verifying the inferences of $E$.

Our explorations are only preliminary, and more experimentation is needed to support the claim that ordinary Euclidean inferences can be checked effi- 
ciently. Moreover, performance can be sensitive to the choice of language and the formulation of the axioms. For example, we were surprised to find that performance was reduced when we replaced our strict "between" predicate with a nonstrict one (presumably because many additional facts, like between $(a, a, b)$, were generated). Thus the data which we report is only suggestive.

We emphasize that the point of these explorations is to show that it should be possible to verify, automatically, proof texts which closely approximate the proofs in the Elements. From the point of fully automated geometric reasoning, our methods are fairly simplistic. There are currently at least four approaches to proving geometric theorems automatically. The first is to translate the theorem to the language of real closed fields and use decision procedures, based on cylindrical algebraic decomposition [10, for the latter; but, in practice, this is too slow even for very simple geometric theorems. A second method, known as Wu's method [65], similarly translates geometric statements into algebraic problems and uses computational algebraic techniques. The method is stunningly successful at verifying many difficult geometric theorems, but it cannot handle the order relation between magnitudes, or the "between" predicate for points on a line; and so it is inadequate for much of the Elements. It is also limited to statements that can be translated to universal formulas in the language of fields. A third method, known as the area method [9, has similar features. Finally, there are so-called "synthetic methods," which use heuristic proof search from geometric axioms. Our methods fall under this heading, but are not very advanced. One would expect to do better with intelligent heuristics and more efficient representations of diagrammatic information, along the lines described by Chou, Gao, and Zhang [9]. (See also [8 for an overview of the various methods.)

In other words, our work does not constitute a great advance in automated geometric theorem proving, even for the kinds of theorems one find in the Elements. Our methods show how to verify the smaller, diagrammatic inferences in Euclid's proofs, given the higher-level structure, and, most importantly, the requisite construction. It is an entirely different question as to how a system might be able to find such a construction automatically. We have not addressed this question at all.

We do hope, however, that our analysis of the way that Euclidean reasoning combines metric and diagrammatic components can provide some useful insights towards modeling proof search in structured domains. Rather than model geometry as a first-order axiomatic system, we have taken advantage of specific features of the domain that reduce the search space dramatically. Particularly notable is the way that we understand Euclidean proofs as building up contexts of data (in our case, "diagrammatic information" and "metric information") that can be handled in domain-specific ways. In other words, adding objects "to the diagram" and inferring metric consequences means adding information to a database of local knowledge that will be accessed and used in very particular ways. We expect that such approaches will be fruitful in modeling other types of mathematical reasoning as well. 


\section{Conclusions}

We conclude by summarizing what we take our analysis of Euclidean proof to have accomplished, discussing questions and other work related to our project, and indicating some of the questions and broader issues that our work does not purport to address.

\subsection{Summary of results}

We claim to have a clean analysis of the argumentative structure of the proofs in Books I to IV of the Elements. We tried to make this claim more precise in Section 2 by discussing the features of the Elements that we have tried to model. We have also gone out of our way, in Section 4 to indicate ways in which proofs in our formal system differ from Euclid's.

It is important to keep in mind that modeling the "argumentative structure" of the Elements is not just a matter of modeling the Euclidean entailment relation in semantic or deductive terms, or giving an account of geometric validity. Rather, our goal has been to understand which individual inferences are licensed by Euclidean practice, so that a line-by-line comparison renders our formal proofs close to Euclid's. To the extent in which we have succeeded, this provides a sense in which the proofs in the Elements are more rigorous than is usually claimed. In particular, we have identified precise rules that govern diagrammatic inferences, which are sound relative to modern semantics; and we have shown that, for the most part, Euclid's proofs obey these rules. As a result, the proofs in the Elements now seem to us to be much closer to formal proof texts than almost any other instance of informal mathematics.

In Section 5. we showed that our formal system is sound and complete for an appropriate semantics of ruler and compass constructions. Insofar as our formal system captures Euclidean practice, this shows that the modern semantics provides an accurate characterization of the provable Euclidean theorems.

In Section 66 we described some initial but promising attempts to verify the inferences of $E$ using current automated reasoning technology. Our findings suggest that it should not be difficult to develop a formal proof checker for $E$. This provides further support to our claim that proofs in the Elements are much closer to formal proofs than is usually acknowledged. The way proofs in $E$ organize data into metric and diagrammatic components, each of which is individually more manageable than their union, hints at a strategy that should have broader application to formal verification.

Finally, we emphasize that we have provided a logical analysis, which screens off cognitive, historical, and broader philosophical questions related to diagram use. This is not to deny the importance of such questions. On the contrary, we feel that by fixing ideas and clarifying basic notions, the logical analysis can support the study of diagram use and Euclidean practice. Thus we take our analysis to show how the norms of a mathematical practice can be analyzed on their own terms, in a way that can support broader inquiry. We hope that we have also demonstrated that such analysis can be rewarding, providing us with 
a better understanding of the mathematics itself.

\subsection{Questions and related work}

Our work is situated in a long tradition of axiomatic studies of geometry, from Hilbert to Tarski and through to the present day. Our emphasis is novel, in that we have tried to characterize a particular geometric practice and style of argumentation. In contrast, modern axiomatic studies aim to provide a deeper understanding of geometry in modern terms, focusing, for example, on the dependence and independence of axioms and theorems, the results of dropping or modifying various axioms, and the relationships to other axiomatic systems. We cannot provide an adequate survey of these topics here, but recommend textbooks by Coxeter 11 and Hartshorne 21. (See also the article by Tarski and Givant [58, which surveys the history of geometric studies by Tarski and his students.)

Our project does raise some traditional logical questions, however. For example, our diagrammatic axioms are all universal axioms, and describe a subset of the universal consequences of Tarski's axioms for Euclid's geometry. It would be nice to have a natural semantic characterization of this set of universal sentences. We know that it is a strict subset of the set of universal consequences of affine geometry: Hilbert [22, Chapter V] showed that Desargues' theorem, which is a consequence of affine geometry, cannot be proved in the plane without the axioms of congruence. Also, given that our construction rules are not independent, it would be nice to have a more principled way of generating the list, beyond simply running through the Elements and making a list of the ones that Euclid seems to use. Finally, as we have mentioned, the question as to the decidability of the $\forall \exists$ consequences of Tarski's axioms, and hence the decidability of $E$, remain open.

Read as first-order axioms, all the basic rules of $E$ are given by universal formulas, except for the construction rules, which have $\forall \exists$ form. If we introduce Skolem functions for these axioms, Herbrand's theorem implies that any theorem of $E$ can be witnessed by an explicit construction involving these functions, together with "if ... then ... else" statements involving atomic conditions. This provides one sense in which Euclidean geometry is "constructive." However, conditional expressions are undesirable; from a constructive perspective, for example, it may be impossible to determine whether a point is actually on a line or only very close to it. Jan von Plato 61 provides a strictly constructive version of affine geometry (see also [62]). Michael Beeson [3] characterizes the problem nicely by observing that Euclid's constructions are not continuous in the input data, and offers a constructive version of Euclidean geometry.

Our project also gives rise to computational questions. On the theoretical side, there is, of course, the problem of providing sharp upper and lower bounds on the complexity of recognizing the various types of inference that, according to $E$, Euclid sanctions as immediate. The challenge of obtaining practical implementations should give rise to interesting problems and solutions as well.

The implementation of a proof-checker for $E$ could be used to help teach 
Euclidean geometry, and Euclidean methods of proof. There are a number of graphical software packages in existence that support geometric exploration and reasoning, of which the best known are perhaps the Geometer's Sketchpad 25], Cabri [64, and Cinderella [18. These systems do not, however, focus on teaching geometric proof. Others have explored the use of graphical front ends to conventional proof assistants, supported by specialized decision procedures for geometry. As we were completing a draft of this paper, we came across Narboux [41, which not only provides a thorough survey of such work, but also describes an impressive effort, Geoproof, along these lines. Even though Geoproof is not based on an explicit analysis of Euclidean proof, it is interesting to note that its primitives and construction rules bear a striking similarity to ours.

\subsection{Broader issues}

In the end, what is perhaps least satisfying about our analysis is that we do not go beyond the logical and computational issues: we provide a detailed description of the norms governing Euclidean proof without saying anything at all about how those norms arose, or why they should be followed. We will therefore close with just a few words about some of the cognitive, historical, and more broadly philosophical issues that surround our work.

On the surface, it might seem that there is a straightforward cognitive explanation as to why some of Euclid's diagrammatic inferences are basic to geometric practice, namely, that these inferences rely on spatial properties that are "hardwired" into our basic perceptual faculties. In other words, thanks to evolution, we have very good faculties for picking out edges and surfaces in our environment and inferring spatial relationships; and these are the kinds of abilities that are needed to support diagrammatic inference. But one should be wary of overly simplistic explanations of this sort; see the discussion in [1]. In particular, one should keep in mind that mature mathematical behavior is only loosely related to more basic perceptual tasks. For instance, the example discussed in Section 2.3 shows that Euclidean geometric reasoning requires keeping in mind that only some features present in a diagram are essential to the mathematical context it is supposed to illustrate. Informal experimentation on some of our nonmathematical friends and family members shows that the expected response to this exercise is by no means intuitively clear; in other words, there seems to be a learned mathematical component to the normative behavior. At the same time, we do not doubt that a better understanding of our cognitive abilities can help explain why certain geometric inferences are easier than others. It would therefore be nice to have a better understanding of the cognitive mechanisms that are involved in such reasoning.

We hope that our analysis can support a refined historical understanding as well. Historians will cringe at our naive claim to have analyzed "the text of the Elements"; there is a long and complicated history behind the Elements, and we have focused our attention on only one translation (Heath's) of one version of the text (Heiberg's). We do expect that, for the most part, our findings are 
robust across the various editions. In fact, some features of the historical record nicely support our claims. Saito [50] has compared the diagrams in a number of editions of the Elements, and has noted that earlier versions exhibit some striking differences from the modern ones. For example, earlier diagrams are often "overspecified": a parallelogram mentioned in the statement of a theorem may be depicted by a rectangle, or even a square. This sits well with our claim that angle and metric information is never inferred from the diagram; the fact that the metric information in the diagrams is so blatantly misleading can be viewed as a subtle reminder to the reader that it should not be relied upon 15 On the other hand, if it turns out that there are ways in which our analysis does not hold up well across historical developments, we expect that our work can help clarify the nature of the historical changes.

Moreover, we hope our analysis can help support a better historical understanding of the evolution of geometric reasoning, and the relationship between different geometric practices. There have been rich historical analyses of the problems and methods found in the ancient geometric tradition [26, 44, as well as, say, the transition to the analytic tradition of Descartes [6. Ken Manders has remarked to us that diagrams are used in fundamentally different ways in nineteenth century projective geometry texts; as the diagrams get more complicated, more of the burden of keeping track of the information they represent is shifted to the text. We expect that the type of analysis we carry out here can complement the historical study, and sharpen our understanding of the mathematical developments.

Finally, there is hope that the rules of Euclidean proof can be "explained" or "justified" not by cognitive or historical data, but, rather, by broader epistemological considerations. For example, Marco Panza [45] takes Euclidean practice to inform a metaphysical account of the nature of geometric objects; Marcus Giaquinto [19] takes cognitive data to support epistemological conclusions regarding the role of visualization in mathematics (but see the critique in [1]); and Jamie Tappenden [56] explores ways of treating visualization as an "objective" feature of mathematics, rather than merely a cognitive device. It is possible that a suitably abstract characterization of our cognitive abilities or the spatial situations the practice tries to model can provide an informative sense in which our fundamental inferences are the "right" ones for the task.

Kant famously took the fundamental principles of geometry to provide synthetic knowledge, grounded by our a priori intuition of space:

Take the proposition that with two straight lines no space at all can be enclosed, thus no figure is possible, and try to derive it from the concept of straight lines and the number two; or take the proposition that a figure is possible with three straight lines, and in the same way try to derive it from these concepts. All of your effort is in vain, and you see yourself forced to take refuge in intuition, as indeed geometry always does. You thus give yourself an object in intuition; but what kind is this, is it a pure a priori intuition or an empirical one?

\footnotetext{
${ }^{15}$ We are grateful to Anthony Jones and Karine Chemla for this observation.
} 
If it were the latter, then no universally valid, let alone apodictic proposition could ever come from it: for experience can never provide anything of this sort. You must therefore give your object a priori in intuition, and ground your synthetic proposition on this. 24, A47-A48/B64-B65].

Indeed, his discussion of Euclid's Proposition I.32 in the Transcendental Doctrine of Method [24, A712-A725/B740-753] provides an illuminating account of how he takes such synthetic reasoning to work. Kant's views on geometry have been studied in depth; see, for example, [17, 52, 53, 54. Lisa Shabel writes:

[The] Kantian account of informal but contentful axioms of Euclidean geometry stemming directly from an a priori representation of space is itself consistent with Euclidean practice: neither Euclid's elements nor its eighteenth-century analogs offer formal axioms but rather definitions and postulates which, if taken seriously, provide a mereotopological description of the relations among the parts of the euclidean plane. The content of these relations is ... precisely what Kant alleges is accessible to us in pure intuition, prior to geometric demonstration. [54, p. 213]

This provides us with a convenient way of framing our project: we have provided a logical description of the mereotopological relations that are implicit in Euclid's definitions and postulates, without feigning hypotheses as to their origins. As Shabel's remarks suggest (see also [54, footnote 4] and [52]), it would be interesting if one could describe a more fundamental account of spatial intuition that can serve to justify or explain the rules of our system. Stewart Shapiro has suggested to us that it would also be interesting to explain what distinguishes Euclid's axioms and postulates from everything he does not say, that is, the assumptions and rules of inference that we take to be implicit in the Elements.

In Section 1, we noted that philosophers have historically been concerned with the problem of how the particular diagrams in the Elements can warrant general conclusions. In particular, a central goal of Kant's account 24, A712A725/B740-753] is to explain how singular objects given in intuition can provide general knowledge. Jeremy Heis has pointed out to us that a curious feature of our account of Euclidean geometry is that the role of the singular - that is, the particular diagram - drops out of the story entirely; we focus only on the diagrammatic features that are generally valid in a given context, and say nothing about a particular instantiation.

There is a fairly mundane, if partial, explanation of the role that concrete diagrams play in geometric practice. Although not every feature found in a particular diagram will be generally valid, the converse is more or less true: any generally valid consequence of the diagrammatic hypotheses will be present in a sufficiently well-drawn diagram. A particular diagram can therefore serve as a heuristic guide, suggesting candidates for diagrammatic consequences that are, perhaps, confirmed by other forms of reasoning. Mumma's original system, Eu, 
is more faithful to this understanding of diagram use; for example, the prover can label a point of intersection in a particular diagram associated with a proof, independent of the mechanisms that are invoked to justify the fact that the intersection is present in general. Some systems of automated reasoning rely on crude procedures to search for possible proof candidates, and then employ other methods to check and fill in the details (see, for example, [34, 60]). It therefore seems to us worth noting that diagram use in mathematics raises two separate issues: first, how (or whether) alternative, nonpropositional representations of mathematical data can be used to facilitate or justify inferences; and, second, how overspecific or imperfect representations can be used to support the reasoning process. Leitgeb [28] begins to address the first issue.

As the vast literature on the Elements indicates, Euclidean geometry has been a lively source of questions for scholars of all persuasions for more than

two millennia. We only hope that the understanding of Euclidean proof we present here will prove useful in furthering such inquiry.

\section{References}

[1] Jeremy Avigad. Review of Visual Thinking in Mathematics, by Marcus Giaquinto. To appear in Philosophia Mathematica.

[2] Clark Barrett and Cesare Tinelli. CVC3. In Computer Aided Verification (CAV) 2007, pages 298-302, 2007.

[3] Michael Beeson. Constructive Euclidean geometry. To appear in the Proceedings of 10th Asian Logic Conference, Kobe, Japan, 2008.

[4] George Berkeley. Principles of Human Knowledge, reprinted in David Armstrong, editor, Berkeley's Philosophical Writings. Macmillian Publishing Company, 1965.

[5] Alexander Bockmayr and Volker Weispfenning. Solving numerical constraints. In Robinson and Voronkov [49], pages 751-842.

[6] Henk J. M. Bos. Redefining geometrical exactness: Descartes' transformation of the early modern concept of construction. Springer, New York, 2001.

[7] Samuel R. Buss. An introduction to proof theory. In Samuel R. Buss, editor, The Handbook of Proof Theory, pages 1-78. North-Holland, Amsterdam, 1998.

[8] S.C. Chou and X.S. Gao. Automated reasoning in geometry. In Robinson and Voronkov [49], pages 707-750.

[9] S.C. Chou, X.S. Gao, and J.Z. Zhang. Machine Proofs in Geometry. World Scientific, Singapore, 1994. 
[10] George E. Collins. Quantifier elimination for real closed fields by cylindrical algebraic decomposition. In Automata Theory and Formal Languages, pages 134-183. Springer, Berlin, 1975.

[11] H. S. M. Coxeter. Introduction to Geometry. John Wiley \& Sons Inc., New York, second edition, 1969.

[12] David Krantz, R. Duncan Luce, Patrick Suppes, and Amos Tversky. Foundations of Measurement, volumes I and II. Academic Press, New York, 1971.

[13] Leonardo Mendonça de Moura and Nikolaj Bjørner. Z3: An efficient SMT solver. In Tools and Algorithms for the Construction and Analysis of Systems (TACAS) 2008, pages 337-340, 2008.

[14] Edward Dean. In Defense of Euclidean Proof. Master's thesis, Carnegie Mellon University, 2008.

[15] Nachum Dershowitz and David A. Plaisted. Rewriting. In Robinson and Voronkov 49, pages 535-607.

[16] Euclid. The Thirteen Books of the Elements, volumes I-III. Dover Publications, New York, second edition, 1956. Translated with introduction and commentary by Sir Thomas L. Heath, from the text of Heiberg. The Heath translation has also been issued as Euclid's Elements: All Thirteen Books Complete in One Volume, Green Lion Press, Santa Fe, 2002.

[17] Michael Friedman. Kant's theory of Geometry. Philosophical Review 94:455-506, 1985. Revised version in Michael Friedman, Kant and the Exact Sciences, Harvard University Press, Cambridge, 1992.

[18] Jürgen Richter Gebert and Ulrich H. Kortenkap. The Interactive Geometry Software Cinderella. Springer, Berlin, 1999.

[19] Marcus Giaquinto. Visual Thinking in Mathematics: an Epistemological Study. Oxford University Press, Oxford, 2007.

[20] William Mark Goodwin. Kant's Philosophy of Geometry. PhD thesis, U. C. Berkeley, 2003.

[21] Robin Hartshorne. Geometry: Euclid and beyond. Springer, New York, 2005.

[22] David Hilbert. Grundlagen der Geometrie. In Festschrift zur Feier der Enthüllung des Gauss-Weber Denkmals in Göttingen. Teubner, Leipzig, 1899. Translated by Leo Unger as Foundations of Geometry, Open Court, La Salle, 1971. Ninth printing, 1997.

[23] Thomas W. Hungerford. Algebra. Springer, New York, 1974. 
[24] Immanuel Kant. Kritik der reinen Vernunft. First edition 1781, second edition 1787. Translated and edited by Paul Guyer and Allen W. Wood as Critique of Pure Reason, Cambridge University Press, Cambridge, 1998.

[25] Key Curriculum Press, editor. The Geometer's Sketchpad: Student Edition. Key Curriculum, 2002.

[26] Wilbur Richard Knorr. The Ancient Tradition of Geometric Problems. Birkhäuser, Boston, 1985.

[27] Gottfireid Leibniz. New Essays Concerning Human Understanding. Open Court Publishing, La Salle, Illinois, 1949.

[28] Hannes Leitgeb. On formal and informal provability. Preprint.

[29] Danielle Macbeth. Diagrammatic reasoning in Euclid's Elements. Preprint.

[30] Paolo Mancosu. Philosophy of Mathematics and Mathematical Practice in the Seventeenth Century. Oxford, New York, 1996.

[31] Kenneth Manders. Diagram-based geometric practice. In Paolo Mancosu, editor, The Philosophy of Mathematical Practice, pages 65-79. Oxford University Press, Oxford, 2008.

[32] Kenneth Manders. The Euclidean diagram. In Paolo Mancosu, editor, The Philosophy of Mathematical Practice, pages 80-133. Oxford University Press, Oxford, 2008. MS first circulated in 1995.

[33] Zohar Manna and Calogero G. Zarba. Combining decision procedures. In 10th Anniversary Colloquium of UNU/IIST, pages 381-422, 2002.

[34] Jia Meng and Lawrence C. Paulson. Translating higher-order clauses to first-order clauses. Journal of Automated Reasoning, 40:35-60, 2008.

[35] Nathaniel Miller. Euclid and his Twentieth Century Rivals: Diagrams in the Logic of Euclidean Geometry. CSLI, Stanford, 2008. Based on Miller's $2001 \mathrm{PhD}$ thesis, "A diagrammatic formal system for Euclidean geometry," Cornell University.

[36] G. Morrow, editor. Proclus: a Commentary on the First Book of Euclid's Elements. Princeton University Press, Princeton, 1970.

[37] Ian Mueller. Philosophy of Mathematics and Deductive Structure in Euclid's Elements. MIT Press, Cambridge, MA, 1981.

[38] John Mumma. Proofs, pictures, and Euclid. To appear in Synthese.

[39] John Mumma. Intuition Formalized: Ancient and Modern Methods of Proof in Elementary Geometry. PhD thesis, Carnegie Mellon University, 2006.

[40] John Mumma. Review of Euclid and his Twentieth Century Rivals, by Nathaniel Miller. Philosophia Mathematica, 16:256-264, 2008. 
[41] Julien Narboux. A graphical user interface for formal proofs in geometry. Journal of Automated Reasoning, 39:161-180, 2007.

[42] Sara Negri. Contraction-free sequent calculi for geometric theories with an application to Barr's theorem. Archive for Mathematical Logic, 42:389-401, 2003.

[43] Sara Negri and Jan von Plato. Cut elimination in the presence of axioms. Bulletin of Symbolic Logic, 4:418-435, 1998.

[44] Reviel Netz. The Shaping of Deduction in Greek mathematics: a Study of Cognitive History. Cambridge University Press, Cambridge, 1999.

[45] Marco Panza. The twofold role of diagrams in Euclid's plane geometry. Preprint.

[46] Moritz Pasch. Vorlesungen über neuere Geometrie. Teubner, Leipzig, 1882.

[47] Giuseppe Peano. I principii di Geometria, logicamente esposti. Bocca, Turin, 1889.

[48] Virgile Prevosto and Uwe Waldmann. Spass+T. In Geoff Sutcliffe, Renate Schmidt, and Stephan Schulz, editors, Empirically Successful Computerized Reasoning (ESCoR) 2006, pages 18-33, 2006.

[49] Alan Robinson and Andrei Voronkov, editors. Handbook of Automated Reasoning. Elsevier Science, Amsterdam, 2001.

[50] Ken Saito. A preliminary study in the critical assessment of diagrams in Greek mathematical works. SCIAMVS, 7:81-44, 2006.

[51] Stephan Schulz. E - a brainiac theorem prover. Journal of AI Communications, 15:111-126, 2002.

[52] Lisa Shabel. Mathematics in Kant's Critical Philosophy: Reflections on Mathematical Practice Routledge, New York, 2003.

[53] Lisa Shabel. Kant's philosophy of mathematics. In Paul Guyer ed., The Cambridge Companion to Kant, second edition, Cambridge University Press, Cambridge, 2006.

[54] Lisa Shabel. Kant's "argument from geometry." Journal of the History of Philosophy, 42:195-215, 2004.

[55] Howard Stein. Eudoxus and Dedekind: on the ancient Greek theory of ratios and its relation to modern mathematics. Synthese, 84:163-211, 1990.

[56] Jamie Tappenden. Proof style and understanding in mathematics I: visualization, unification, and axiom choice. In Paolo Mancosu, Klaus Frøvin Jorgensen, and Stig Andur Pedersen, editors, Visualization, Explanation and Reasoning Styles in Mathematics, pages 147-214. Springer, Berlin, 2005. 
[57] Alfred Tarski. What is elementary geometry? In Leon Henkin, Patrick Suppes, and Alfred Tarski, editors, The Axiomatic Method: with Special Reference to Geometry and Physics, pages 16-29. North-Holland, 1st edition, 1959.

[58] Alfred Tarski and Steven Givant. Tarski's system of geometry. Bulletin of Symbolic Logic, 5:175-214, 1999.

[59] A. S. Troelstra and Helmut Schwichtenberg. Basic Proof Theory. Cambridge University Press, Cambridge, second edition, 2000.

[60] Robert Veroff. Solving open questions and other challenge problems using proof sketches. Journal of Automated Reasoning, 27:157-174, 2001.

[61] Jan von Plato. The axioms of constructive geometry. Annals of Pure and Applied Logic, 76:169-200, 1995.

[62] Jan von Plato. A constructive theory of ordered affine geometry. Indagationes Mathematicae, 9:549-562, 1998.

[63] Christoph Weidenbach, Renate Schmidt, Thomas Hillenbrand, Rostislav Rusev, and Dalibor Topic. System description: Spass version 3.0. In Frank Pfenning, editor, CADE-21: 21st International Conference on Automated Deduction, pages 514-520, Springer, Berlin, 2007.

[64] Wendy Wilgus. Exploring the Basics of Geometry with Cabri. Texas Instruments, 1998.

[65] Wen Tsün Wu. Mechanical Theorem Proving in Geometries. Springer, Vienna, 1994. Translated from the 1984 Chinese original by Xiao Fan Jin and Dong Ming Wang.

[66] Martin Ziegler. Einige unentscheidbare Körpertheorien. L'enseignement mathématique, 28:269-280, 1982. Unpublished translation by Michael Beeson. 